\title{
PRELIMINARY ANALYSIS OF LIP-WING CONCEPT USING COMPUTATIONAL FLUID DYNAMICS AND ACTUATOR DISC THEORY
}

\author{
By \\ Peter Walker \\ Bachelor of Engineering, Ryerson University 2013 \\ A thesis, \\ presented to Ryerson University \\ in partial fulfillment of the \\ requirements for the degree of \\ Master of Applied Science \\ in the Program of Aerospace Engineering \\ Toronto, Ontario, Canada, 2016 \\ (C)(Peter Walker) 2016
}




\section{AUTHOR'S DECLARATION}

I hereby declare that I am the sole author of this thesis. This is a true copy of the thesis, including any required final revisions, as accepted by my examiners.

I authorize Ryerson University to lend this thesis to other institutions or individuals for the purpose of scholarly research.

I further authorize Ryerson University to reproduce this thesis by photocopying or by other means, in total or in part, at the request of other institutions or individuals for the purpose of scholarly research.

I understand that my thesis may be made electronically available to the public 


\title{
Abstract
}

\section{PRELIMINARY ANALYSIS OF LIP-WING CONCEPT USING COMPUTATIONAL FLUID DYNAMICS AND ACTUATOR DISC THEORY}

\author{
Peter Walker \\ A thesis for the degree of \\ Master of Applied Science, 2016 \\ Department of Aerospace Engineering, Ryerson University
}

This thesis presents a preliminary analysis of the lip wing concept proposed by Dusan Stan of Aliptera Aircraft. A inviscid CFD-CAD actuator disk model was used to simulate a comparable geometry to that which was investigated experimental by Aliptera Aircraft. In general, a 10\%$12 \%$ increase in thrust was produced at an optimal lip wing angle of $30^{\circ}$. This increase in thrust was consistent with the experimental results obtained by Aliptera Aircraft. These preliminary results are promising and encourage further research. 


\section{Acknowledgements}

It is said that Sir Isaac Newton claimed that if he had seen a little further than his fellow man, it was only by standing on the shoulders of giants. It is in this spirit that the author would like to acknowledge the tremendous debt owed to the faculty and staff of Ryerson University's Aerospace Engineering Department, and particularly to his advisor, Dr. Jeffrey Yokota, who gave me this opportunity and stuck it out with me through the swamps, deserts and chasms along the way.

Whatever I may make of myself, the opportunities I have found at Ryerson will be as much responsible as my own labours.

Mente et Artificio, 


\section{Table of Contents}

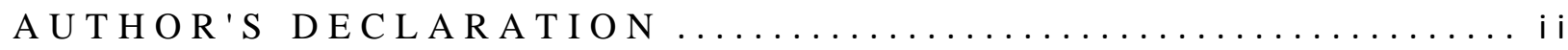

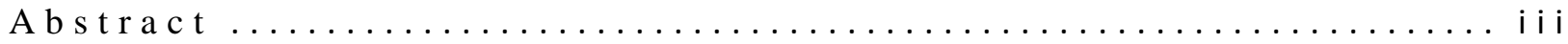

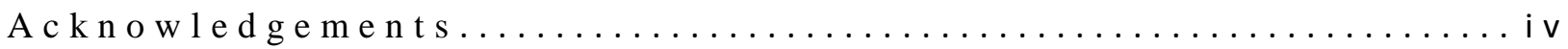

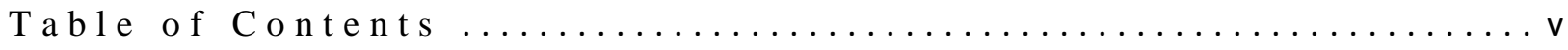

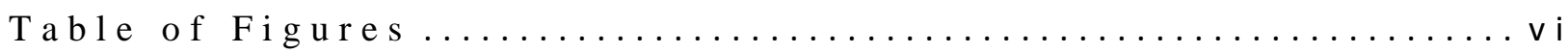

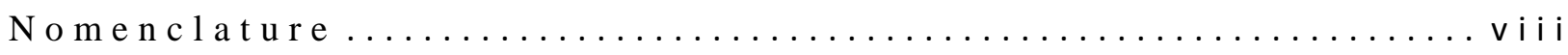

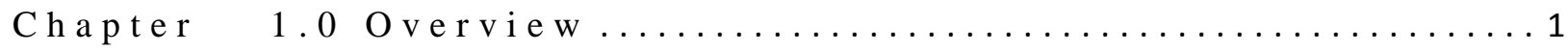

Chapter 2.0 Introduction and Theory $\ldots \ldots \ldots \ldots \ldots \ldots \ldots \ldots \ldots$

2.1 Thrust and Momentum Equations ................

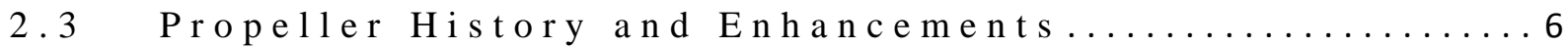

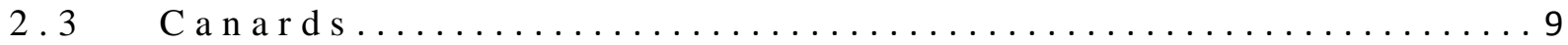

2.4 Computational Fluid Dynamics ...................

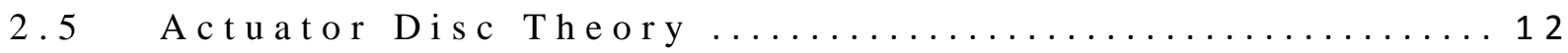

2.6 Lip Wing Concept and Preliminary Work .......... 15

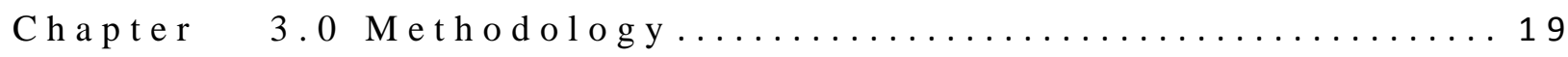

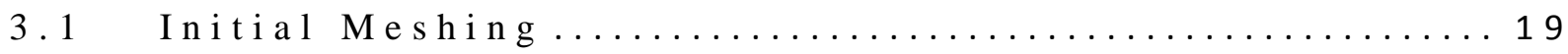

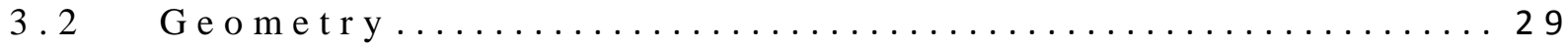

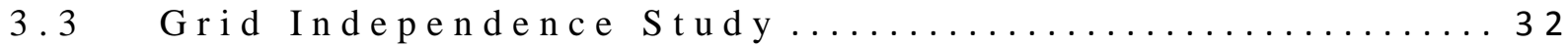

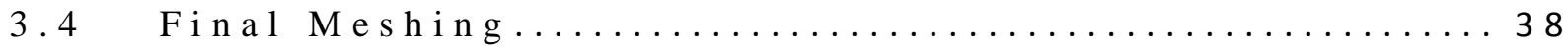

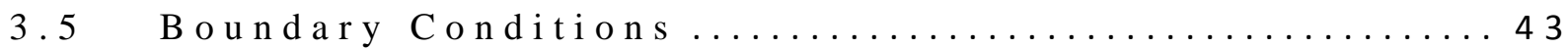

3.6 Solution Data Collection ..................... 5

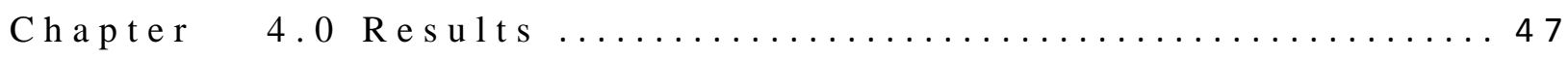

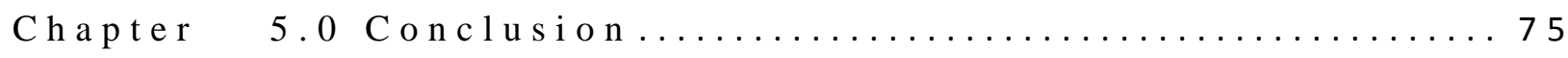

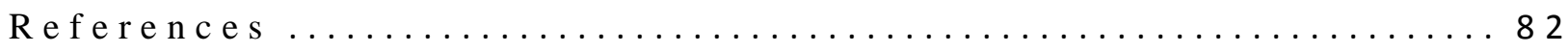




\section{Table of Figures}

Figure 1 Saab 37 Viggen Jet Fighter [Courtesy of Swedish Air

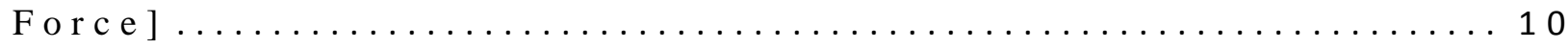
Figure 2 Custer Wing Channel Experiment [Courtesy of NASA]

Figure 3 Element Quality $2 \sigma$ Below Mean in Improved Geometry

Figure 4 Original Control Volume Geometry, Element quality

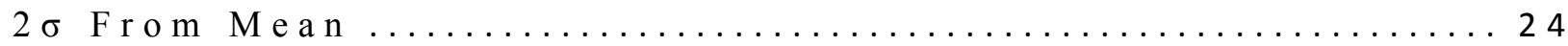

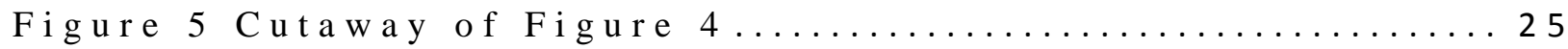

Figure 6 Distribution of Elements in Cylindrical Control

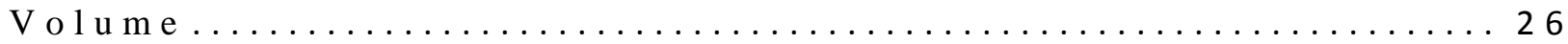

Figure 7 Distribution of Elements $\sigma$ Below Mean Quality ...2 27

Figure 8 Shroud with Lip Geometry Constraints ........... 29

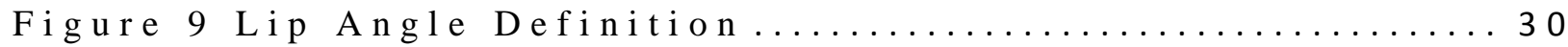

Figure 10 Isometric View of $10^{\circ}$ Lip Angle Final Geometry.. 31

Figure 11 Side View of $10^{\circ}$ Lip Angle Final Geometry...... 32

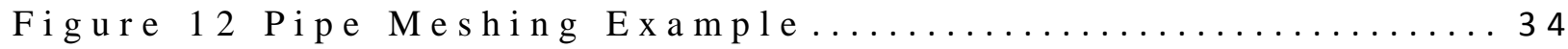

Figure 13 Actuator Disc and Control Volume Geometry..... 36

Figure 14 Normalized Thrust of Actuator Disc Alone as

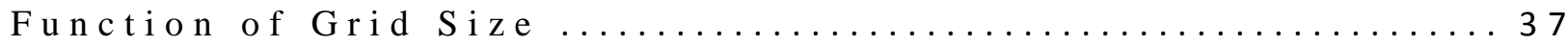

Figure 15 Example of Numerical Diffusion at Outlet for $1 \mathrm{st}$

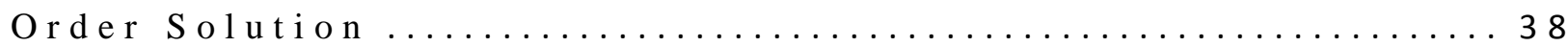

Figure 16 Element Breakdown of Mesh, / .................. 99

Figure 17 Element Breakdown of Mesh, $\alpha=10^{\circ} \ldots \ldots \ldots \ldots \ldots \ldots 40$

Figure 18 Average Element Quality of Models ...........4 1

Figure 19 Average Element Quality as Function of Lip Wing

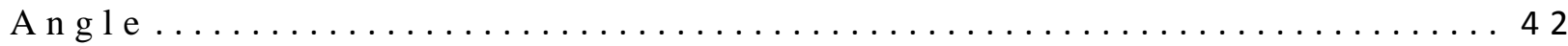

Figure 20 Distribution of Elements By Element Quality ....4 3

Figure 21 Effect of Lip Angle on Thrust ................47

Figure 22 Normalized Thrust as a Function of Grid Size With

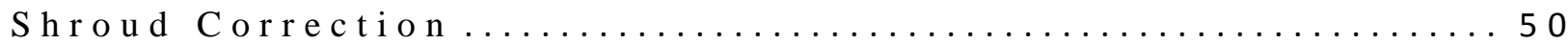

Figure 23 Effect of Lip Angle on Thrust (Shroud Normalized)52

Figure 24 Residual Behavior for $\alpha=20^{\circ} \ldots \ldots \ldots \ldots \ldots \ldots \ldots \ldots \ldots . \ldots 6$

Figure 25 Residual Behavior for $\alpha=40^{\circ} \ldots \ldots \ldots \ldots \ldots \ldots \ldots \ldots \ldots 57$

Figure 26 Residual Behavior for $\alpha=60^{\circ} \ldots \ldots \ldots \ldots \ldots \ldots \ldots \ldots \ldots . \ldots 5$

Figure 27 Effect of Lip Angle on Change in Mass Flux.....62

Figure 28 Effect of Lip Angle on Mean Velocity.........65

Figure 29 Mean Inlet Velocity at Different Lip Angles.....66

Figure 30 Mean Outlet Velocity at Different Lip Angles ....67

Figure 31 Dynamic Pressure Contours of Lip Wing $\left(\alpha=20^{\circ}\right)$

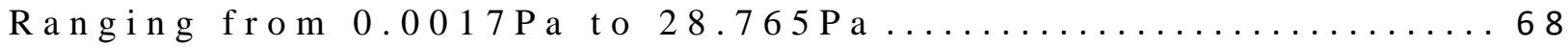


Figure 32 Dynamic Pressure Contours of Lip Wing $\left(\alpha=40^{\circ}\right)$

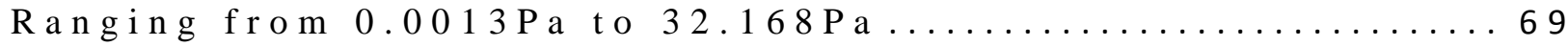

Figure 33 Dynamic Pressure Contours of Lip Wing $\left(\alpha=60^{\circ}\right)$

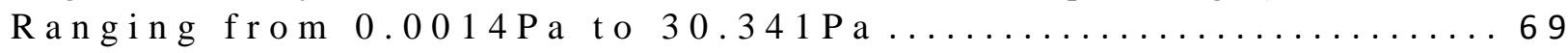

Figure $34 \mathrm{Comparison} \mathrm{of} \mathrm{Streamlines,} \alpha=40^{\circ}$ (top) and $\alpha=60^{\circ}$

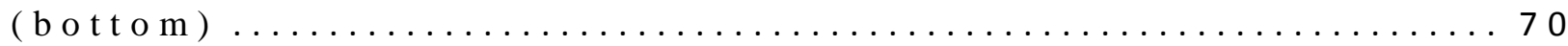

Figure $35 \mathrm{Streamlines}$ Outlet, $\alpha=20^{\circ} \ldots \ldots \ldots \ldots \ldots \ldots \ldots \ldots \ldots \ldots$

Figure $36 \mathrm{Streamlines} \mathrm{Outlet,} \alpha=40^{\circ} \ldots \ldots \ldots \ldots \ldots \ldots \ldots \ldots \ldots \ldots 72$

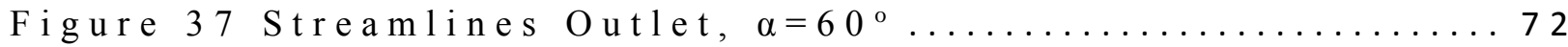

Figure 38 Normalized Areas of Max Velocity at Outlet, Black ( Shroud Alone), Light Red $\left(\alpha=20^{\circ}\right)$ Light Green, $\left(\alpha=40^{\circ}\right)$ and

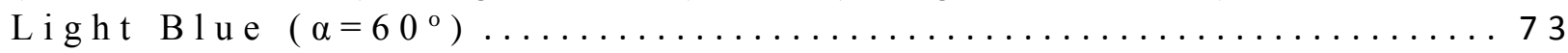

Figure 39 Geometry of Shroud and Separated Lip-Wing.....77

Figure 40 Streamline Diagram of Separated Lip and Shroud..79 


\section{Nomenclature}

RANS Equations: Reynolds Averaged Naiver Stokes Equations

CFD: Computational Fluid Dynamics

SIMPLE Algorithm: Semi-Implicit Method for Pressure Linked Equations Algorithm

SIMPLEC Algorithm: Semi-Implicit Method for Pressure Linked Equations Corrected

Algorithm

SIMPLER Algorithm: Semi-Implicit Method for Pressure Linked Equations, Revised Algorithm

$\alpha$ : Lip Wing Angle

$\rho$ : Density

A: Area

$\mathrm{V}:$ Speed

$\Delta \mathrm{V}$ : Change in Speed

$\dot{m}$ : Mass flow Rate

$\vec{V}$ : Velocity

n: normal

t: time

h: height

D: Drag

W: Weight

F: Force

P: Pressure

VTOL: Vertical Take Off and Landing

STOL: Short Take Off and Landing

NACA: National Advisory Committee for Aeronautics 


\section{Chapter 1.0 Overview}

The goal of this thesis is to present the background, methodology and results of a preliminary analysis using computational fluid dynamics (CFD) to evaluate the lip wing concept proposed by Dusan Stan of Aliptera Aircraft. Preliminary work done by Aliptera Aircraft suggests that the lip wing concept could provide a considerable improvement to the thrust available to aircraft using ducted propellers. Due to the small size of Aliptera Aircraft, the company did not have the in-house expertise or resources to investigate the nature or cause of this effect. Some experimental work has been done, however the experimental methodology was crude, and further exploration using such apparatus would be difficult.

As a preliminary step towards validating this concept, a computational fluid dynamic model was created using CATIA CAD software and ANSYS FLUENT. This CFD model has served as a method to explore the original experimental design in greater depth by examining the flow field using a control volume approach. However, as CFD is a numerically based approach, additional steps, such as validation were first necessary. In addition, use of CFD requires the balancing of computational load and model fidelity.

This thesis will outline the basic relevant theory in both fluid mechanics and computational fluid dynamics in general as well as previous work done with the lip wing concept in particular. The methodology of the model generation will be discussed, with a particular focus 
on discussing grid independence and boundary conditions. The effect of the shroud alone will be investigated as well, and then a more in depth discussion of the flow field itself, looking at streamlines and pressure contours will be performed. Finally, the next logical steps in research will be outlined, including improved modelling for fluid viscosity, transient effects and improved geometry. 


\section{Chapter $2.0 \quad$ Introduction and Theory}

Since the earliest days of aeronautics, thrust has been a key limiter to the design of aircraft, if not the key limiter. Many designs for aircraft which may have aerodynamically feasible or at least were put forward in the latter half of the 1800 s, but due to the low power-toweight ratio of steam powered engines, none of them were capable of controlled, sustained flight. Gliders had been possible for many years before, however the work of the Wright brothers on optimizing propeller was in many ways the final key step in powered, fix winged flight. [Anderson 1]

Because of its incredible importance, the production and efficient use of thrust has been a major focus of aerospace research since the First World War. As difficult as it may be for modern engineers to imagine, there was a time when the key performance characteristics were not understood, nor how they related to quantities like the coefficients of lift, drag and also thrust. As just one of many pertinent examples, the rate of climb for an aircraft is heavily determinate on the thrust available as shown by the equation below,

$$
\frac{d h}{d t}=\left(\frac{T-D}{W}\right) V
$$

\section{Equation 1 Rate of Climb}

Where $\mathrm{T}$ is thrust, $\mathrm{D}$ is drag, $\mathrm{W}$ is weight and $\mathrm{V}$ is the airspeed of the aircraft. Here in this equation, three of the four quantities are directly or indirectly related to thrust, with airspeed and 
drag both being heavily influenced by thrust (i.e. the thrust available determining the speed an aircraft can reach, and also the component of drag caused by airspeed.) [Anderson 1,2]

\subsection{Thrust and Momentum Equations}

At the most fundamental level, thrust can be defined as a change of momentum in a control volume. For an arbitrary control volume, the change in momentum can be given by the following integral equation:

$$
T=\oint_{C S} \rho V(V \cdot n) d A=\sum_{\text {out }} \rho V A \vec{V}-\sum_{\text {in }} \rho V A \vec{V}
$$

Equation 2 Momentum Equation

In a simplified way, this equation merely states that the change in momentum in an arbitrary control volume is the difference between the momentum coming out and the momentum coming in. This may seem like common sense, but there is a caveat. This is not the matter of simple scalar multiplication. Momentum is a vector, and it has a vector component, $\vec{V}$. The equation can actually be simplified still further, as $\rho A V$ is the mass flow rate. [Anderson 2,4]

$$
T=\sum_{\text {out }} \rho V A \vec{V}-\sum_{\text {in }} \rho V A \vec{V}=\sum_{\text {out }} \dot{m} \vec{V}-\sum_{\text {in }} \dot{m} \vec{V}
$$

Equation 3 Change of Momentum Equation 
In a $n^{\text {th }}$ dimensional case, the vector equation can be broken up into $n$ separate equations. In a three-dimensional case, this means the equations can be broken up into the following three equations, covering all possible directions.

$$
F=\sum_{\text {out }} \dot{m} V_{x}-\sum_{\text {in }} \dot{m} V_{x}
$$

Equation 4a: Force Equation in X Direction

$$
\sum_{\text {out }} \dot{m} V_{y}-\sum_{\text {in }} \dot{m} V_{y}
$$

Equation 4b: Force Equation in Y Direction

$$
\sum_{\text {out }} \dot{m} V_{z}-\sum_{\text {in }} \dot{m} V_{z}
$$

Equation 4c: Force Equation in Z Direction 


\subsection{Propeller History and Enhancements}

Considerable interest has been invested since the early days of powered flight to improve the amount of thrust that could be provided by aircraft engines. Propellers of early aircraft were subject to much optimization and intense study. Originally they were thought to function in an analogous method to those used in naval vessels, i.e. that a well-designed naval propeller would be suitable for use in aircraft. One of the most important contributions to aeronautical design that the Wright Brothers made was to break aerodynamicists out of this line of thinking. The Wright

brother's applied research into propeller design for their original flyer showed the importance of viscosity and density on propeller design. Water for example, is about two orders of magnitude more viscous than air and considerably more incompressible (experiencing a reduction in volume of only $1.8 \%$ even at 40MPa of pressure). [Greatrix, Anderson 1, $2 \& 4$ ]

The results of this pioneering work, as well as further follow up studies over the last century are seen in the designs of modern aircraft and navel propellers. Clearly, as a visual inspection of the two types of propellers will reveal, there are similarities but there are also important differences that make each more suitable for their distinct environments. For example, nautical propellers can almost entirely ignore compressibility effects. These similarities however do occasionally lead to designers and researchers pursuing similar concepts to enhance the thrust of propeller based propulsion. [Carrolton] 
One example of this is the propeller shroud or duct. In naval vessels this is generally called a Kort Nozzle, and can function to either accelerate or decelerate the flow in different directions depending on the orientation of the airfoil. The venerable NACA series of airfoils is often used as the profile for the Kort Nozzles. Accelerating Kort nozzles by far the most popular in civilian naval architecture as they increase thrust in the direction of the propeller thrust. It accomplishes this primarily by creating circulation around the nozzle. This circulation creates an inward facing vortex which results in greater thrust. Close tolerance between the propeller and the ducting also impedes the formation of tip vortices which also improves efficiency. Decelerating nozzles result in negative thrust in the direction of the duct using similar principles, which may seem like an odd design choice for a propulsion system, but this can be very useful in suppressing noise, which would factor into the design of a submarine's propeller. [Carrolton]

In the accelerating case, Kort Nozzles are only effective at relatively low speeds, generally less than $20 \mathrm{kph}(\sim 10 \mathrm{knots})$. At higher speeds the drag of the nozzle along with increased cavitation generally are sufficient to nullify any improvement. [Carrolton]

Cavitation is not an issue in the design of aircraft propellers, however ducted fans as they are commonly called are an area of intense interest particularly with Uninhabited Aerial Vehicles (UAVs) and dirigibles. Their principle of operation is generally simpler than those of a Kort Nozzle. They are rarely designed with a complicated airfoil design to create the inner facing vorticities, possibly due to the lower density of air compared to water, which would reduce the momentum produced by such an approach, and the advantage would not be commeasure with the 
increased cost in design and manufacture. With ducted fans the emphasis is more on reducing the effects of tip loss on the propeller. [Ohanian, Piolenc et al., Piolenc et al.]

Tip loss is the result of the non-ideal nature of the propeller. Tip vortices will form at the trailing edge of the blade tips, and the momentum to fuel these vortices comes from the propeller, and thus is reduces the momentum from the corresponding wake which produced useable thrust. By sizing the duct very close to the radius of the propeller, the tight clearance impedes the formation of vortices which can vastly improve propeller efficiency. In turn this can allow for a smaller propeller to produce the equivalent amount of thrust of a larger, un-ducted propeller. Indeed, improvements of efficiency greater than $85 \%$ have been reported using appropriately sized ducts. This tends to be a more popular approach either for VTOL/STOL aircraft, for example the Lockheed Martin F-35 Lightning II, and for model aircraft / UAVs. The shroud also greatly cut down on noise as well as protects the propeller from Foreign Object Debris (FOD) when on the ground. [Ohanian, Ko et al.]

Similar to the Kort Nozzle, the ducted fan remains only a niche application. It adds weight to the system, which is a design drawback for naval craft but is often a serious detriment to aircraft. While it may cause an improvement in the overall thrust to weight ratio of the aircraft, the penalty in weight may be unacceptable to some designers. Additionally, the very tight tolerances required by the ducting will increase both engineering and manufacturing costs, and vibrations will need to be tightly controlled. [Ohanian, Ko et al. Piolenc et al.] 


\subsection{Canards}

Other devices that have been used to optimize an aircraft's ability to fly and climb at greater speeds is the canard, generally described as a small foreplan or forewing placed ahead of the main wing. They were originally used on the very first fixed winged power aircraft, where the name canard originated from the duck like appearance of derivatives of the Wright Brother's craft (canard is the French word for duck) [Anderson 1\&2]

Unlike a propeller shroud, canards improve lift and control, without directly improving thrust. While it did offer better control for the pilot when it was dynamically managed, static canards had some very complex interactions with the surrounding flow field, which had a tendency towards instability. For this reason, interest in canards has fluctuated over the twentieth century. Eventually with the development of computer controlled actuators, canards became quite a popular design feature on a number of fighter jets. The first such aircraft to make use of these structures was the Saab 37 Viggen, produced for the Swedish Airforce who was the sole operator.

Canards, shroud and propellers have all benefitted from the development of computer aided engineering, specifically the development of computational fluid dynamics (CFD), the use of numerical methods and computer algorithms to solve complicated flow fields. 


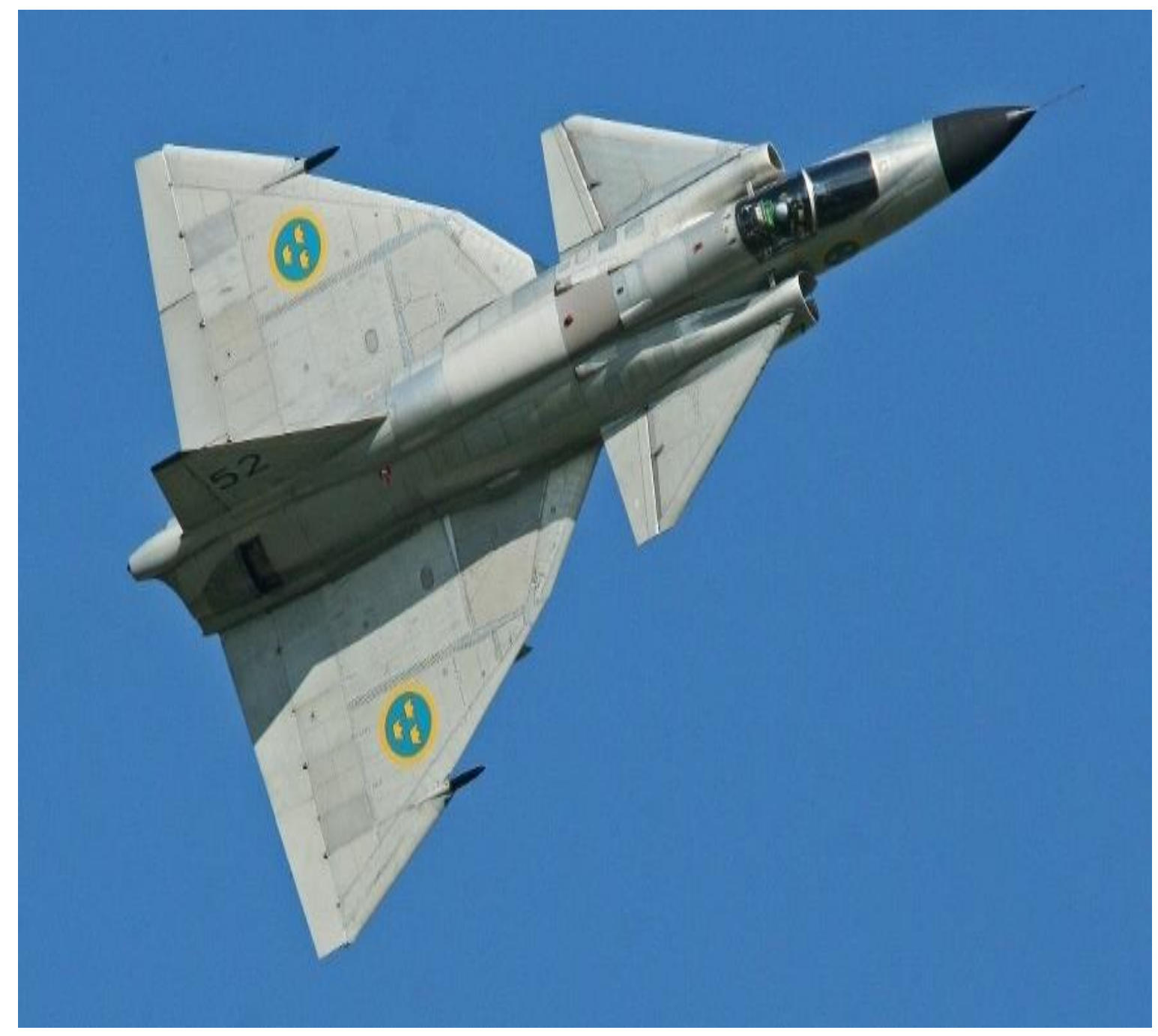

Figure 1 Saab 37 Viggen Jet Fighter [Courtesy of Swedish Air Force]

\subsection{Computational Fluid Dynamics}

The basics of modern CFD are the numerical solutions to the Navier-Stokes equations, which can be used to calculate the energy, momentum and mass flux of a fluid, whether transient or steady state. Different CFD software will use different methods to solve these equations, and an in-depth discussion into the precise nature of FLUENT's is beyond the scope of this work. 
That said there are some similarities that should be discussed to provide a sufficient conceptual framework. CFD software uses a meshing software which will break up a given geometry into discrete elements. The solver will then calculate the results of the equations either within the volume of the element (the finite volume method) or at nodes (finite difference method). Of these two, the finite volume method is much more widely used, due to the greater ease of meshing complex geometry as compared to the finite difference methods. [Ansys Inc, Anderson 3]

CFD can make use of either pressure or density based solvers, though the second type is much more commonly used when compressibility effects are a major factor of the analysis. When using a pressure based solver, the SIMPLE algorithm and its derivatives are among the common types of solvers. The SIMPLE algorithm starts with an initial guess, before proceeding to solve the discretized momentum equations, then moves onto solve for pressure. It iterates over and over again until the changes between iteration reaches the preset convergence criteria, i.e. that the difference between any two iterations is smaller than some threshold. [Ansys Inc, Anderson 3]

As an alternative to the basic SIMPLE algorithm, is the so called SIMPLEC or SIMPLE Consistent algorithm. It's very similar to the SIMPLE algorithm, except with a lower threshold of omission and so can provide greater accuracy, especially when skewness is a major issue in the mesh. Its computational cost is comparable to that of the SIMPLE algorithm, but can result in 
convergence between 10\%-30\% faster than the SIMPLE algorithm, given similar circumstances. [Ansys Inc]

The second common variant of the SIMPLE algorithm is the SIMPLE Revised. Unlike SIMPLE it does not correct the pressure field using the pressure correction equation and only uses this equation to correct the velocities. While more computationally intensive than the SIMPLE algorithm, generally requiring up to 50\% more computational resources, it converges between 30\%-50\% faster than the SIMPLE algorithm alone. [Ansys Inc]

\subsection{Actuator Disc Theory}

CFD modelling allows for something that experiments cannot, the modelling of concepts that are mathematically valid but do not have a physical basis. Using CFD it is a trivial matter to model wholly inviscid flow, which would be impossible to accomplish in a wind tunnel. [Anderson 3]

Actuator disc theory is just such a method: the approximation of a propeller by an infinitely thin disc. In simplest terms this is a pressure discontinuity, as shown previously in figure 5. To the left the pressure is standard atmospheric, to the right of the disc is the higher 
pressure region. This higher pressure region will impart momentum on the propeller via Newton's third law, and thus produce thrust. [Aagaard, Greatrix, Madsen]

The equation behind the actuator disc theory is very simple, and due to this simplicity it suffers from rather low accuracy.

$$
T=A\left(P_{2}-P_{1}\right)
$$

Equation 5: Actuator Disc Thrust Equation

Actuator disc theory doesn't take into account many of the more complex behaviors seen in actual propellers. For this reason, it is only used in very preliminary design studies. But with CFD it can still be a very useful tool for modelling the fluid flow as a whole in a more general way. Whereas the modelling of a full propeller can be quite computationally intensive and requires an in-depth survey of design variables that need to be modelled, an actuator disc can achieve a reasonable degree of accuracy even when modelling the complex interaction between the propeller and an aircraft. [Bettschart, Goorjian, Greatrix]

Work by Roosenboom et al made use of actuator disc theory to model the wake of propellers driven by turboprop engines and then compared the results to sophisticated imaging methods that examined velocities and vorticities. Good experimental results between the numerical results and the experiments were observed by the group, when using Reynolds 
Averaged Naiver-Stokes equation. Their research was using Particle Image Velocimetry, a nonintrusive whole-field velocity measurement technique to analyze the velocity of the field of a whole. This overcame one of the major problems usually found in experimentation. To measure the flow at various points would require probes and instrumentation that could impact the flow. The results of vorticity analysis using both PIV and CFD methods, displayed some similar trends, in for example the shape of the wake's repeating elements.

The use of an actuator disc in this manner compared favorably in terms of computational resources compared to the work of Goble and Hooker, which did similar modelling using full propeller modelling of a P-3C Orion aircraft. That analysis required a supercomputer for the full analysis which resulted in very poor convergence behavior even with a particularly dense unstructured grid. Eventually an actuator disc was used to simplify the calculations and improve convergence behavior.

Actuator disc modelling is also proving quite popular in the modelling of wind and tidal turbines. Batten et al. used an actuator disc and a RANS solver to predict the performance of a tidal turbine. As part of Batten et al's preliminary research, they compared results from several different authors who had also done work using disc models, and compared them to experimental results.

The work of Batten et al. concluded that the actuator disc model has a 97\% accuracy compared to experimental data. This accuracy increased when the control volume had a large 
distance (generally defined as 5 to 7 times greater than the diameter of the disc) between the disc and the outlet.

Crastoa et al. did similar work with wind turbines to Batten et al. with tidal turbines, but with a greater emphasis on wake modelling, particularly interference between the wake and terrain. Actuator disc modelling proved quite accurate even when dealing with this very complex interaction. As with the work of Batten et al. the predictions between the power generated and that of the actuator disc modelling work. Crastoa et al. used a form of inverse actuator disc, where the actuator disc was a momentum sink rather than a source like it would be if modelling a propeller.

\subsection{Lip Wing Concept and Preliminary Work}

The lip wing concept proposed by Dusan Stan of Aliptera Aircraft can be seen as an extension of the channel wing concept that was pioneered by NACA and its successor NASA.[NACA RM L53A09] 


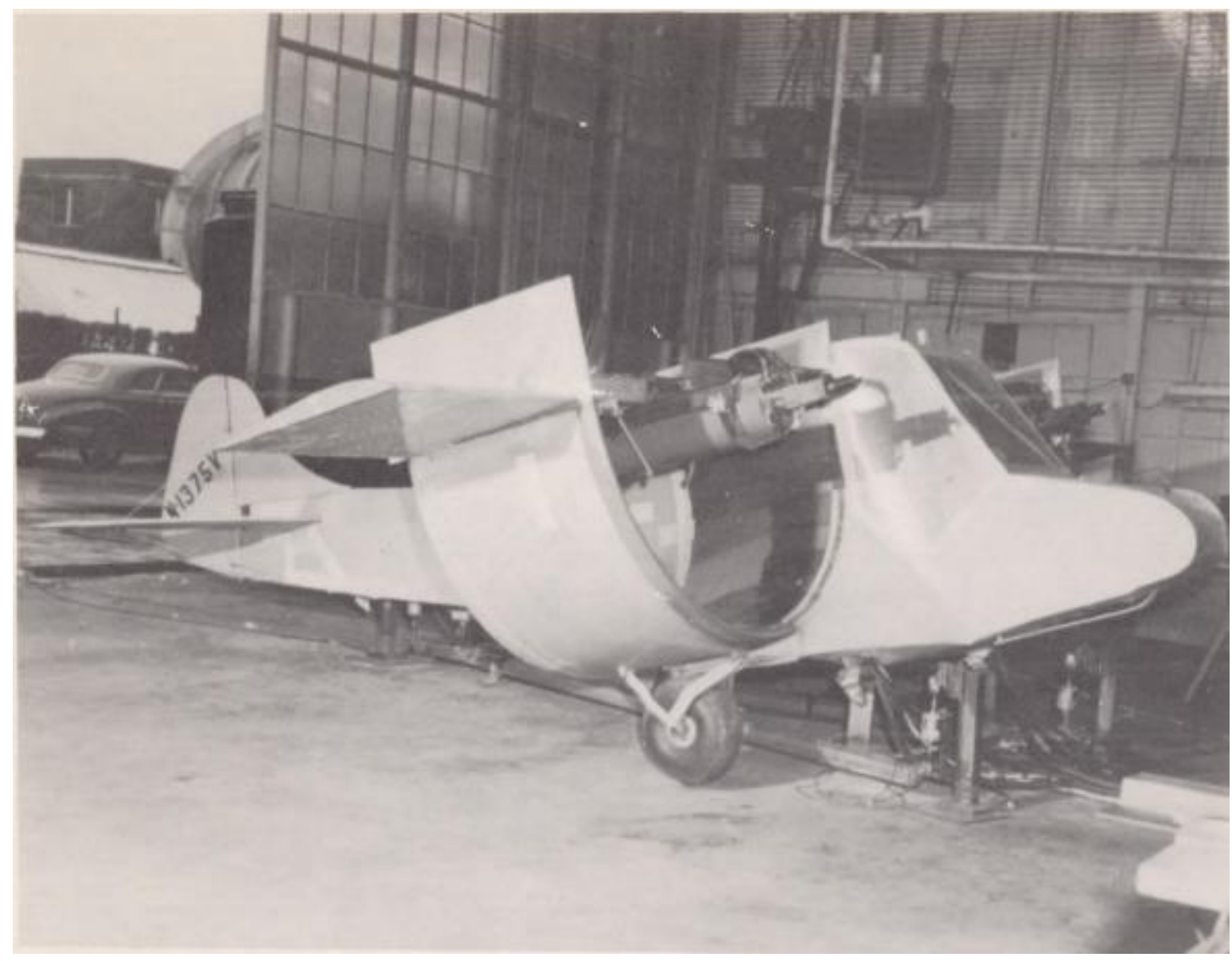

Figure 2 Custer Wing Channel Experiment [Courtesy of NASA]

This configuration was found to generate a resultant force that enhanced both thrust and lift, even when held statically. The aircraft was also observed to have neither trim nor control when using a standard configuration tail. This configuration became known as the Custer channel.

Aliptera aircraft plans to expand this concept into the lip wing, a protrusion from a ducted propeller to produce a similar resultant force. This would allow for the production of personal 
aircraft capable of VTOL/STOL flight. Below is an artist's conception of a proposed Personal Aircraft

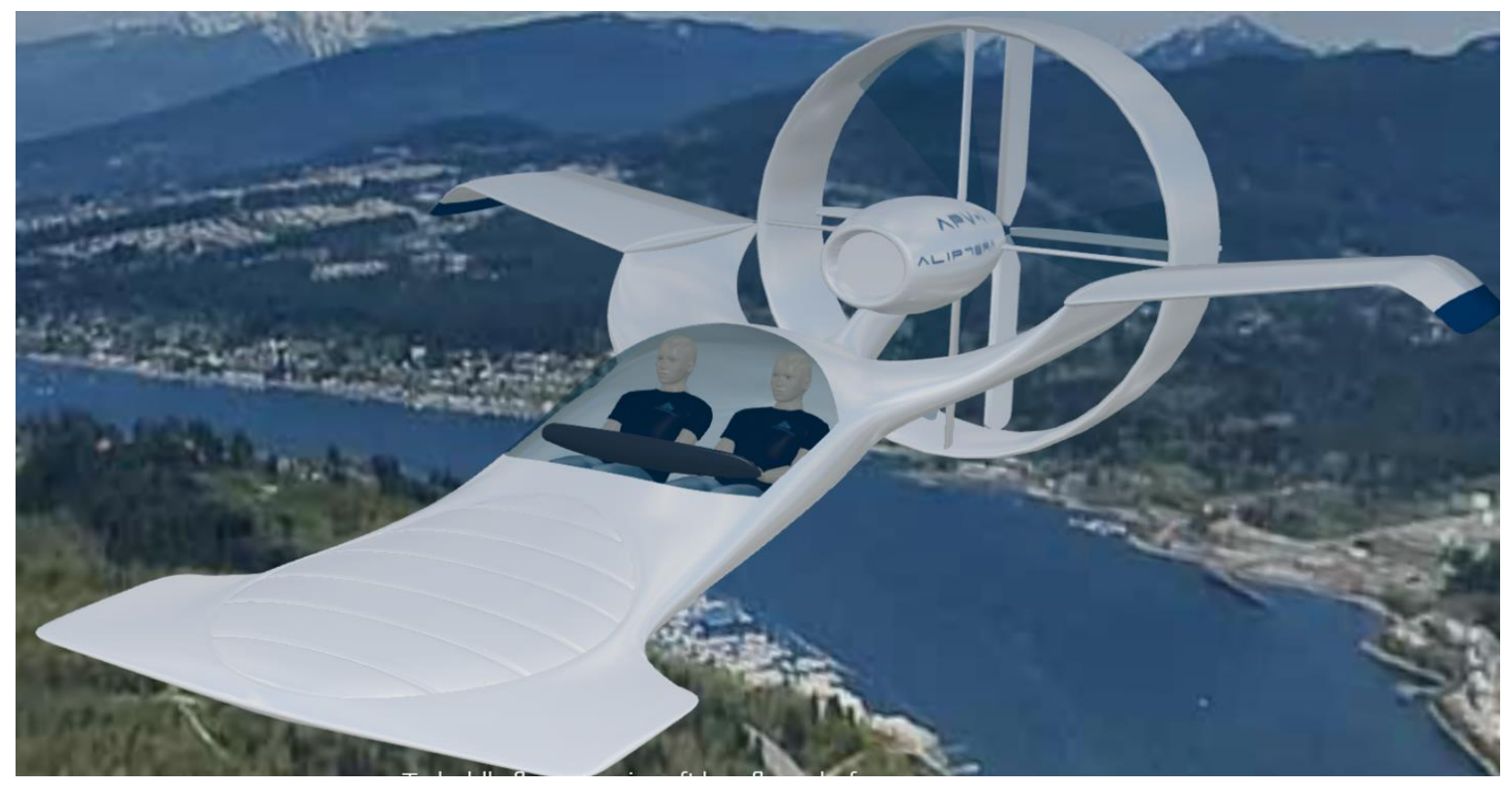

Figure 3 Artist Conception of Aliptera Personal Aircraft, Reprinted with Permission, (C) Aliptera Aircraft

Aliptera performed some experimentation with the lip wing concept using Styrofoam and model aircraft components, specifically a small scale propeller and electric motor. This propeller was first shrouded, which showed a measurable increase in thrust efficiency compared to the baseline of the propeller alone, as would be expected by theory. Finally, a lip wing was attached 
to the shroud as shown in the image below.

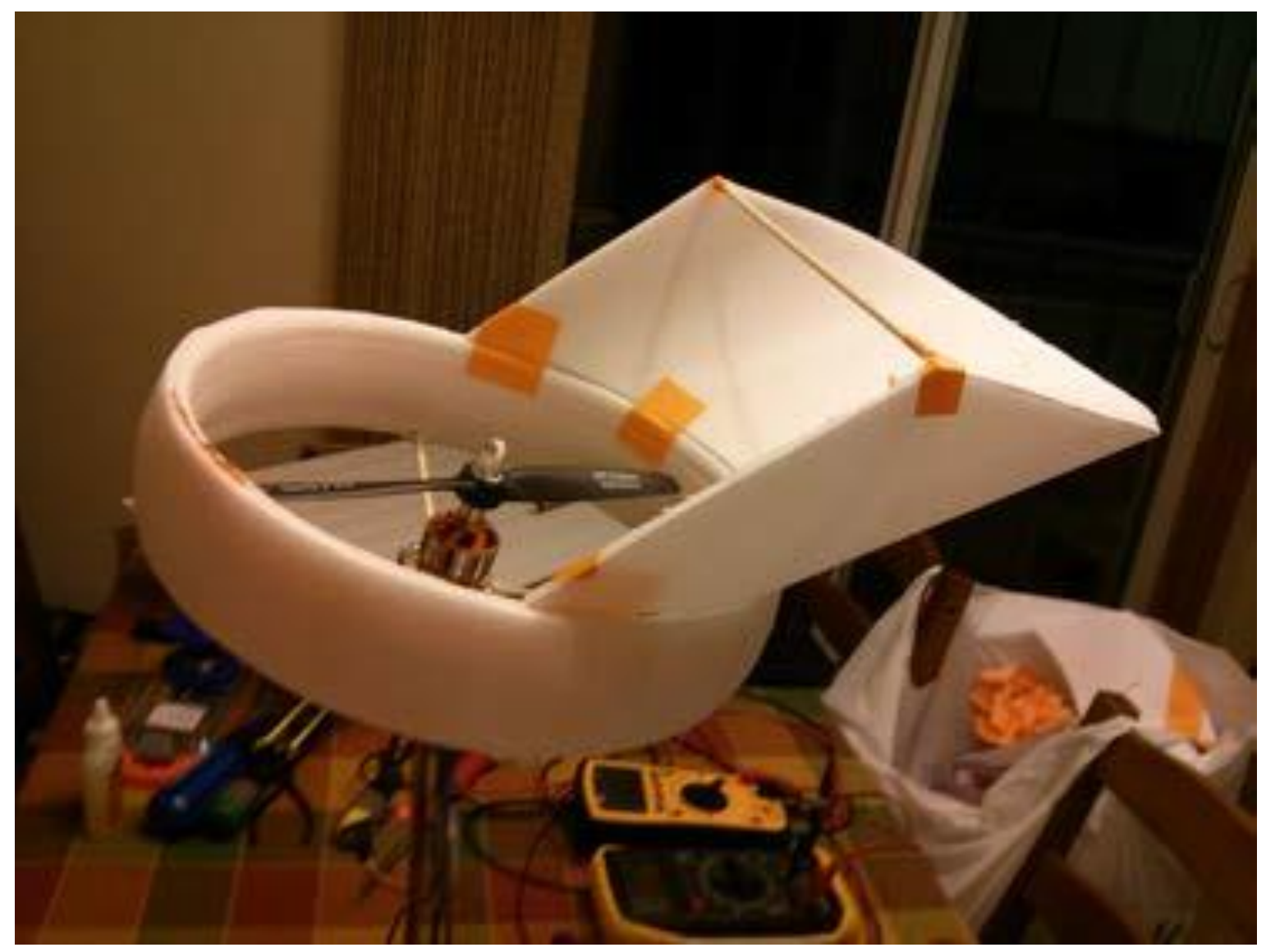

Figure 4 Aliptera Aircraft Experimental Setup. Reprinted with Permission, (c) Aliptera Aircraft

With the lip wing attached, the force measured on the scale was $18 \%$ greater than with the ducted propeller alone. It is from this early experimental geometry that the CAD geometry for the CFD model, so that this experimental work could serve as a baseline. 


\section{Chapter 3.0 Methodology}

\subsection{Initial Meshing}

In order to more closely approximate the results of the original experimental work, the CAD models were designed to serve as close facsimile to the physical models. In order to simplify the geometry, the 8 in propeller was rounded to a $20 \mathrm{~cm}$ diameter, with the shroud having $0.25 \mathrm{~cm}$ of clearance. The creation of the actuator disc required the creation of an additional solid component that would later be defined as a fluid zone within the larger control volume.

Originally, a rather thin cylinder was used, but this proved to cause problems with meshing. Element quality can be an important factor in both the stability and accuracy of CFD models. A sufficient number of poor quality elements can make convergence impossible, or alternatively can lead to a solution which while numerically valid is physically incorrect, e.g. heat spontaneously flowing from low temperature to high temperature areas. The figure below shows all the elements in the original thin cylinder approach that were 3 standard deviations below the average element quality. 


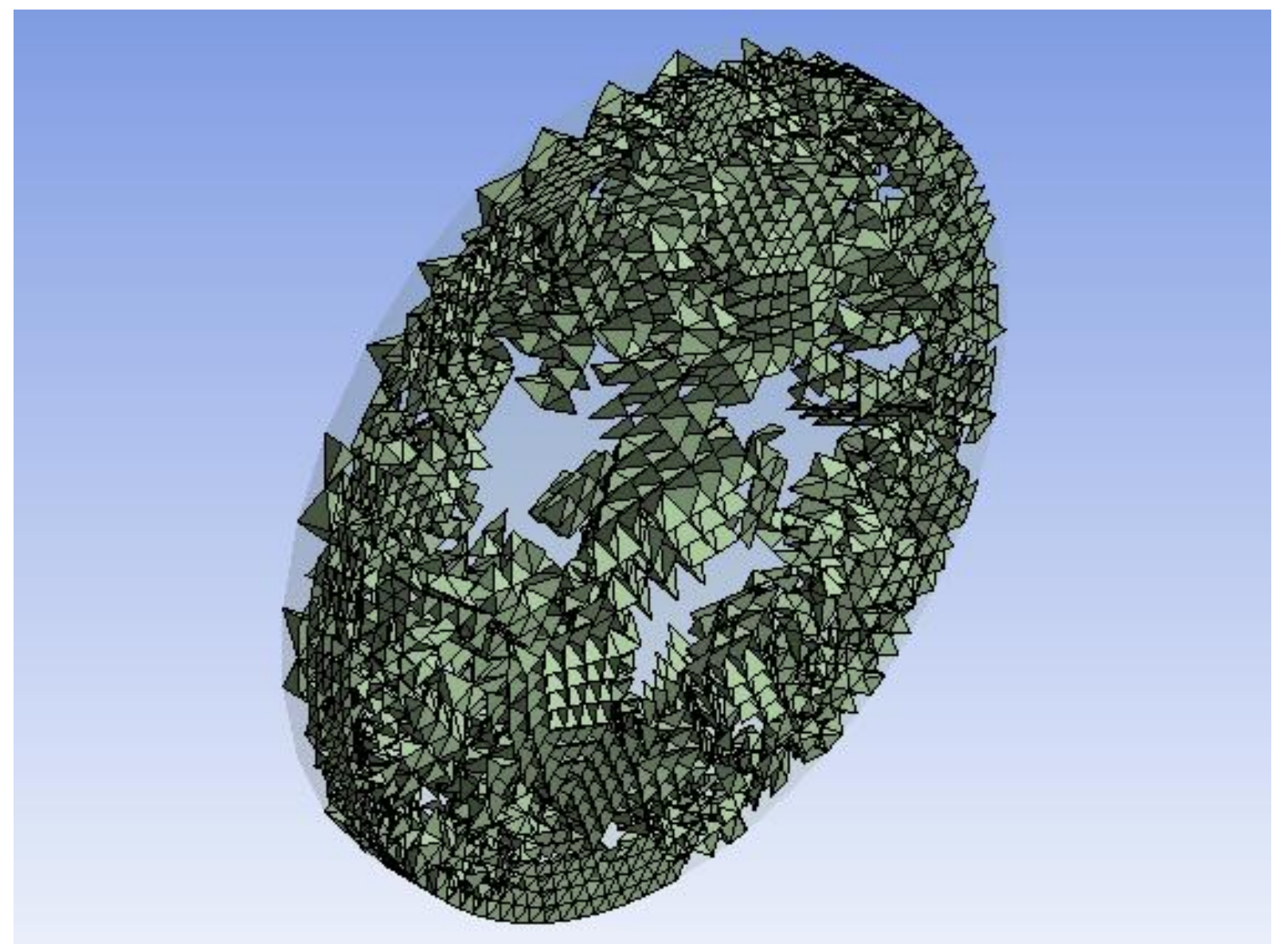

Figure 5 Poor Quality Elements, $3 \sigma$ from Standard Deviation in Early Geometry

Furthermore, there were even quite a few elements that are four standard deviations below average element qualities. These elements in particular are found around the edge of the cylinder. 


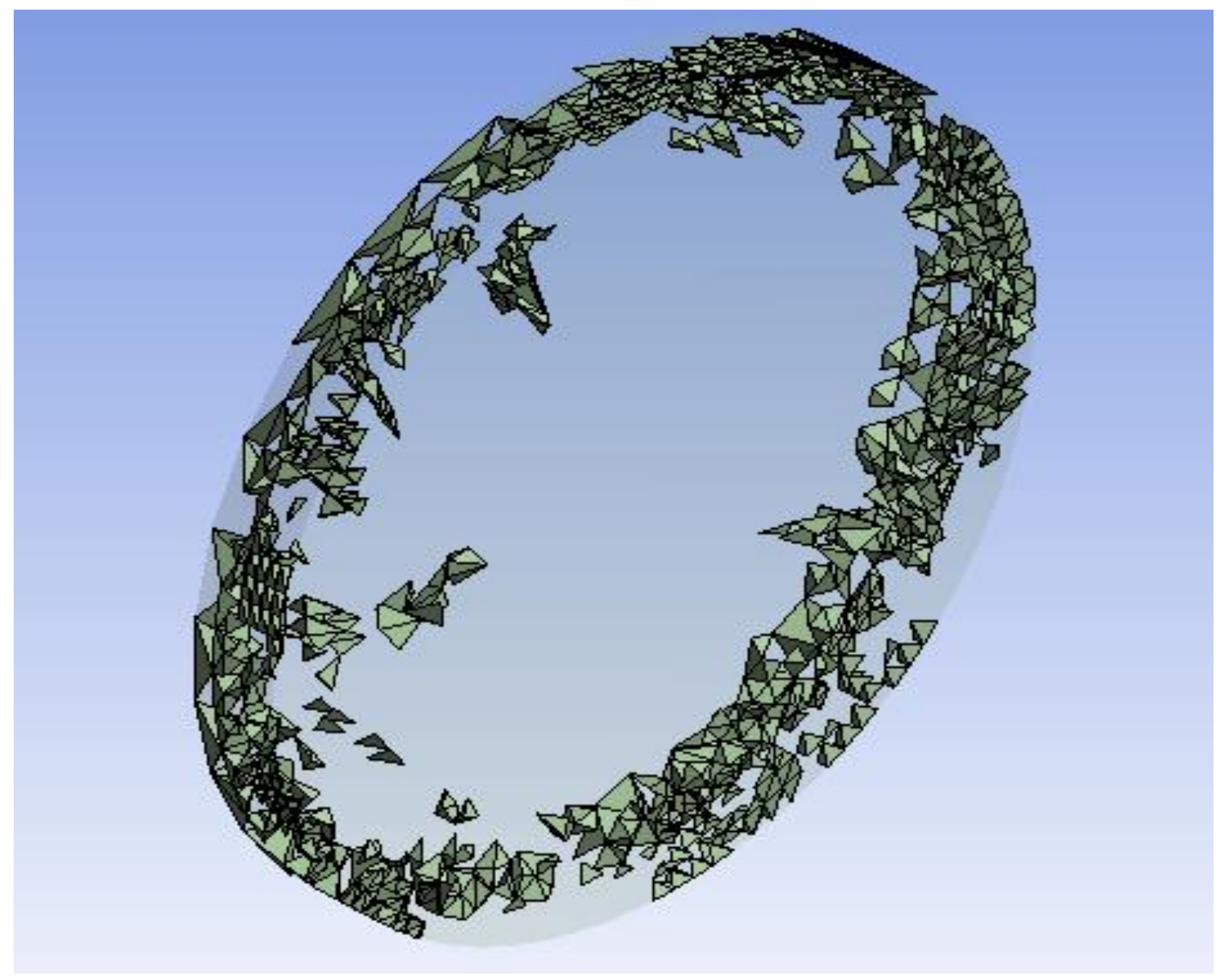

Figure 6 Very poor quality elements, $4 \sigma$ from Mean in Early Geometry

Overlaying element quality map with a map of high skewness elements, it's obvious there's a great deal of overlap between the two, as seen below. 


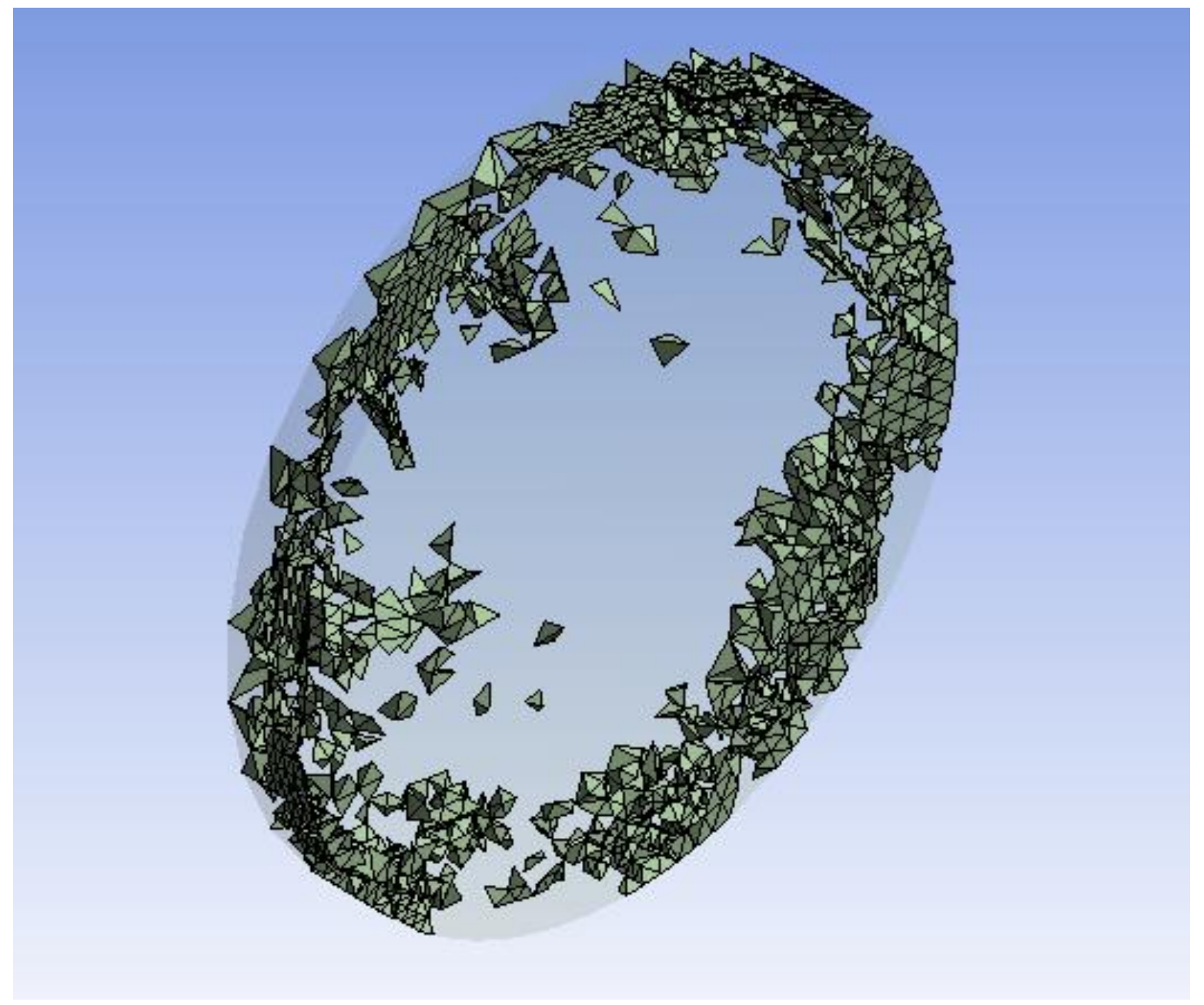

Figure 7 High Skewness Elements in Early Geometry

The solution to this problem was to use a much longer cylinder. This allowed the ANSYS Mesher to more appropriately tailor the elements sizing for reducing skewness. When looking at the mesh of this larger cylinder, only a handful of elements are two standard deviations from the mean, as shown below. In addition, there are no zones of significantly above average skewness (significantly above average in this case being 2 standard deviations above the mean). 


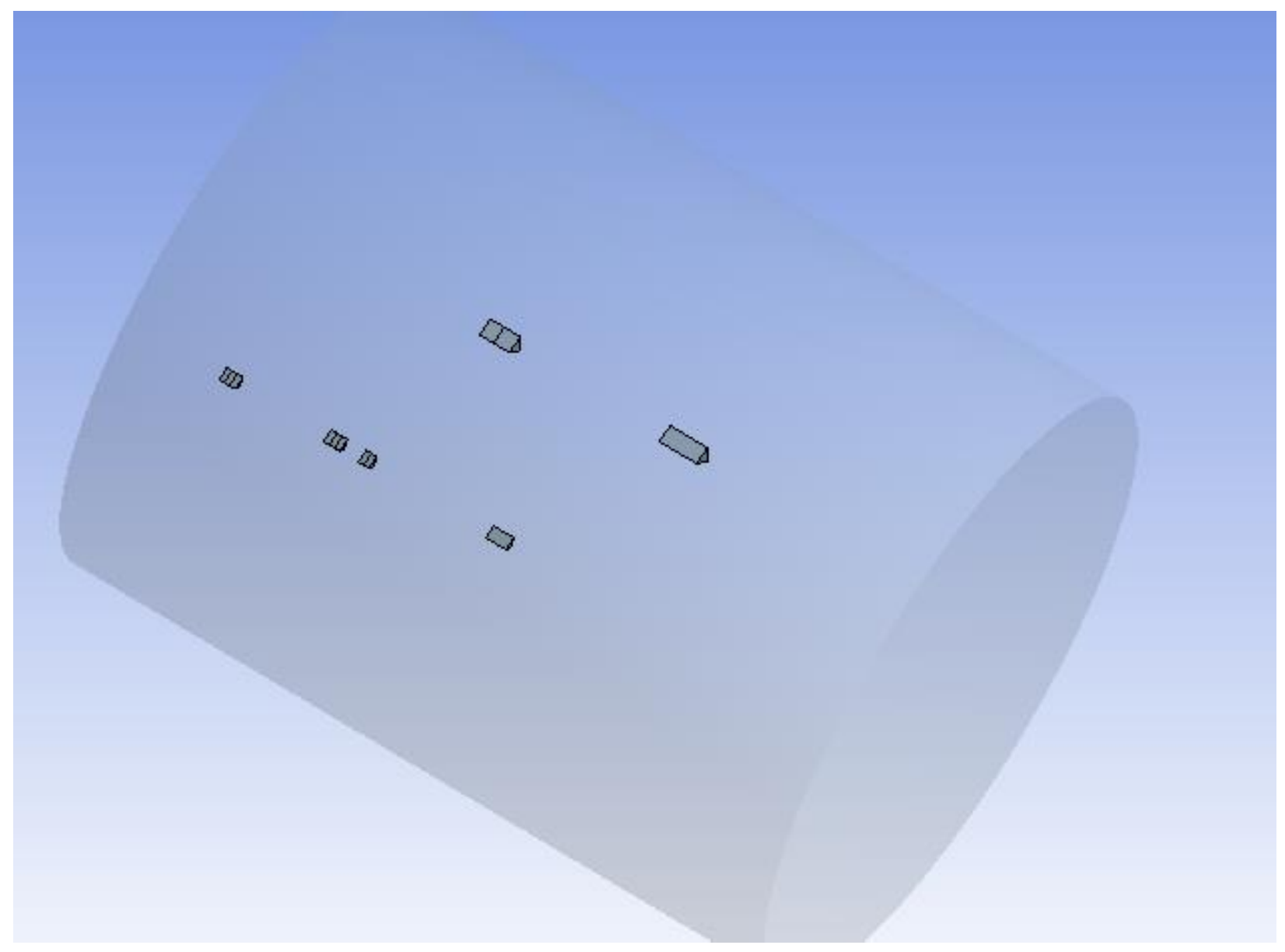

Figure 3 Element Quality $2 \sigma$ Below Mean in Improved Geometry

Initially, it was desirous to achieve an artificially high mesh density in the area of maximum interest, i.e. the lip wing and actuator disk. By creating a higher density around the area of interest, it is possible to maximize the computational load of a dense mesh in the area where it is most useful, rather than solving multiple elements at the areas of the control volume which are of no real interest to this preliminary investigation. 
As a first attempt at increasing the mesh density on areas of interest, an inner sphere was created inside the control volume where mesh refinements would be attached. Unfortunately, the high degree of curvature in the sphere results in very poor levels of element quality inside the spherical region as shown in the figure below. Highlighted are only the elements that are two standard deviations from the mean, which appear to make up the majority of the elements within the region.

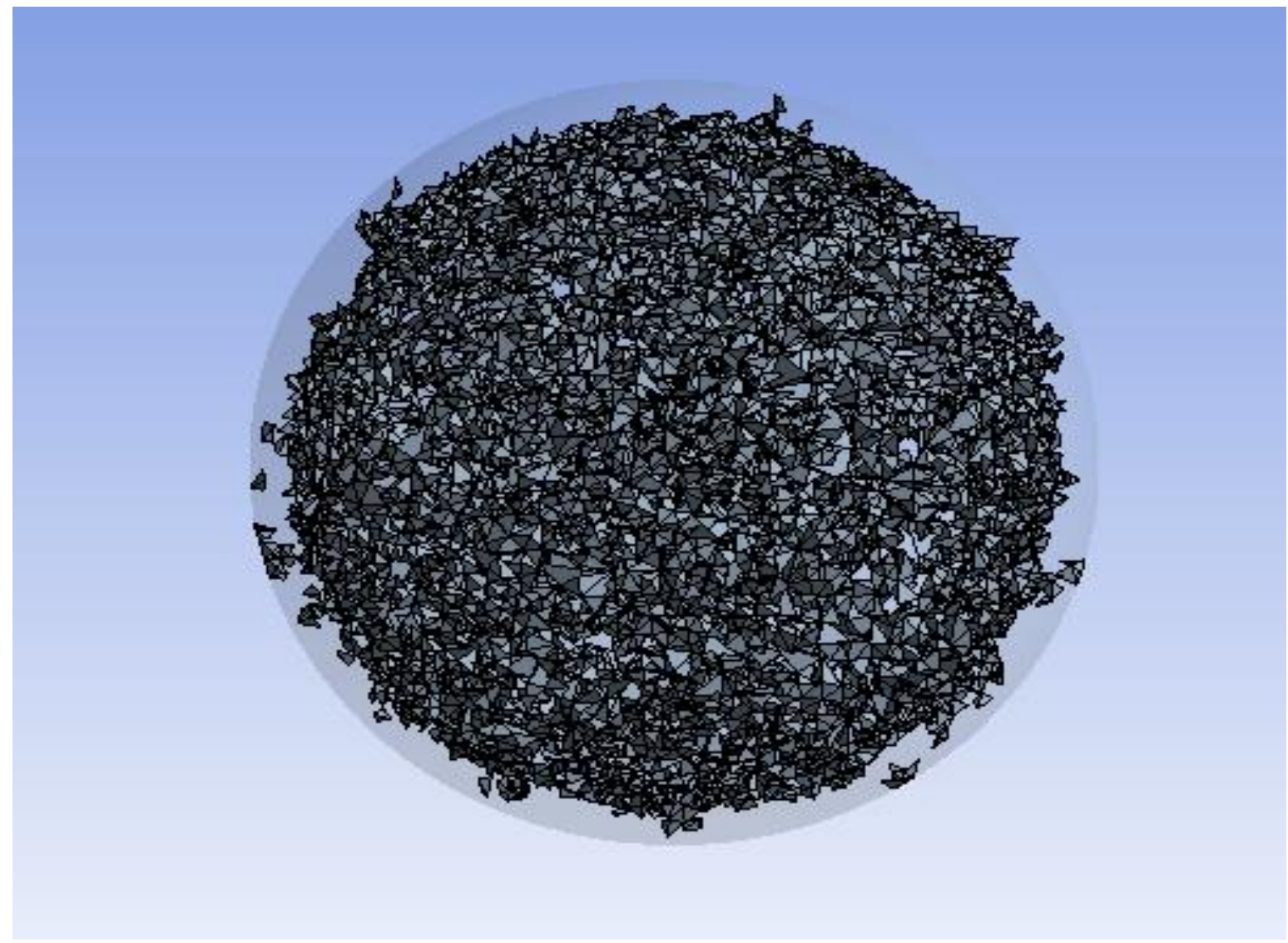

Figure 4 Original Control Volume Geometry, Element quality 2o From Mean

A cut away view of the sphere shows that this is not limited merely to the surface of the sphere.

The poor quality elements progress all the way to the center. 


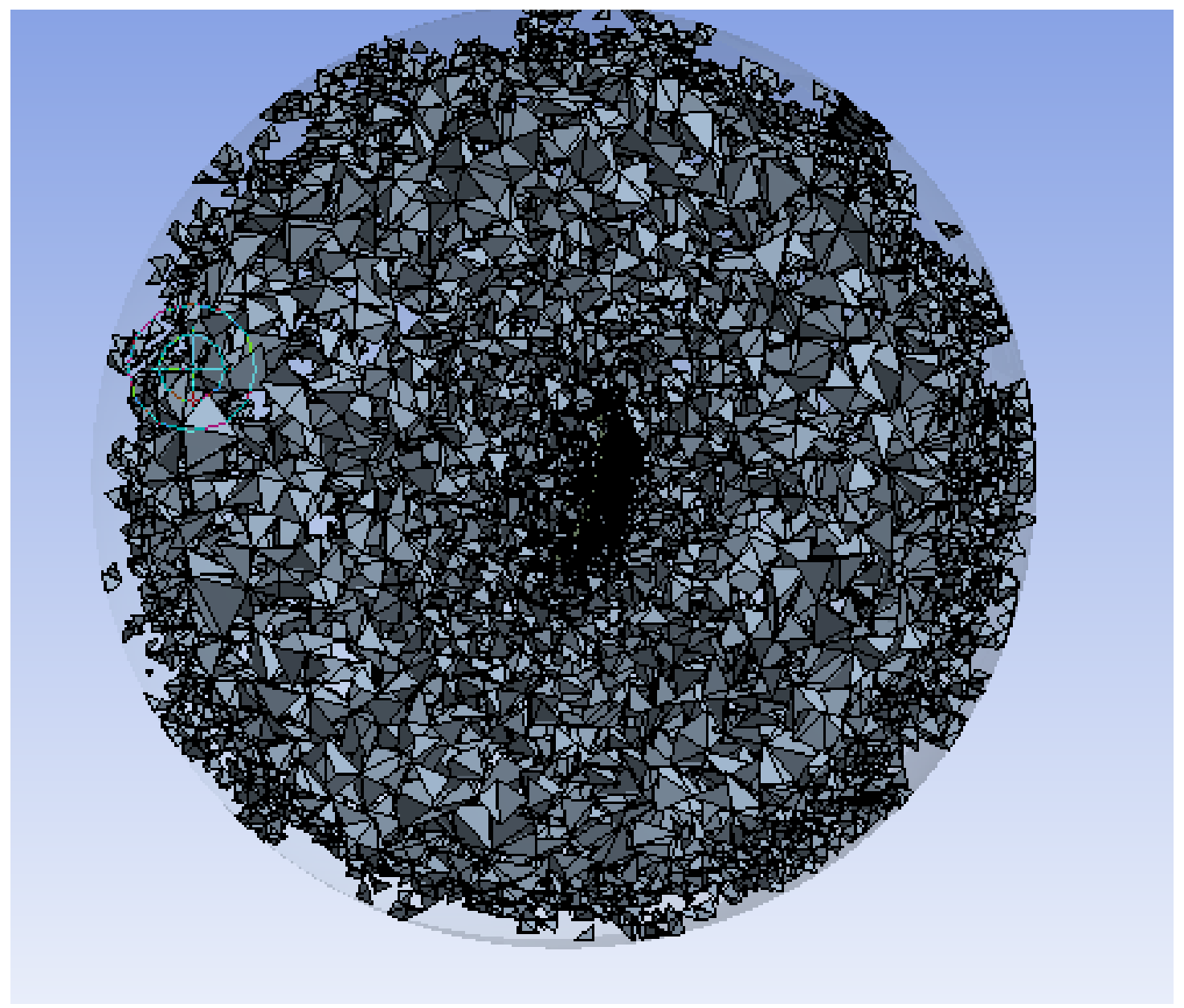

Figure 5 Cutaway of Figure 4

These elements show both high levels of skewness and very poor orthogonal quality.

Orthogonal quality is the measure of the angles normal the face of an element and angles from

the centroid of the element to the faces. Ideally these two sets of angles would be coincident with each other (i.e. $180^{\circ}$ ). Based on these issues, the inner sphere geometry was eventually

abandoned in place of simpler mesh refinements at the actuator disc. This results in a more even distribution of high, average and lower quality elements throughout the control volume, but with higher concentrations of elements around the actuator disc and lip, as shown in the figure below. 


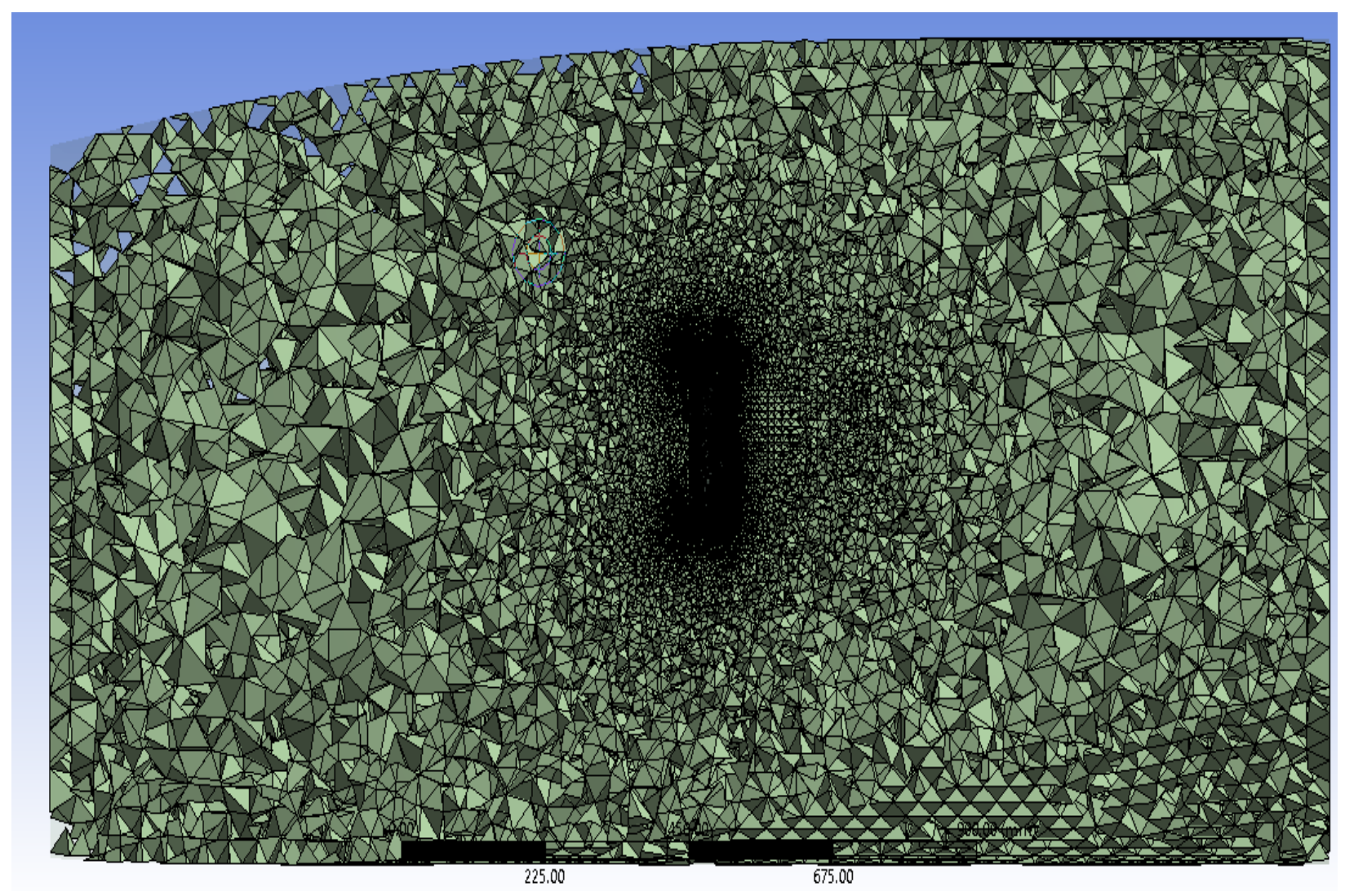

Figure 6 Distribution of Elements in Cylindrical Control Volume

The above cross section highlights both the greater density and smaller size of elements closer to the actuator disc and lip. These elements are among the highest quality of the mesh over all, almost a standard deviation above the average, and are found at all points in the control volume. 


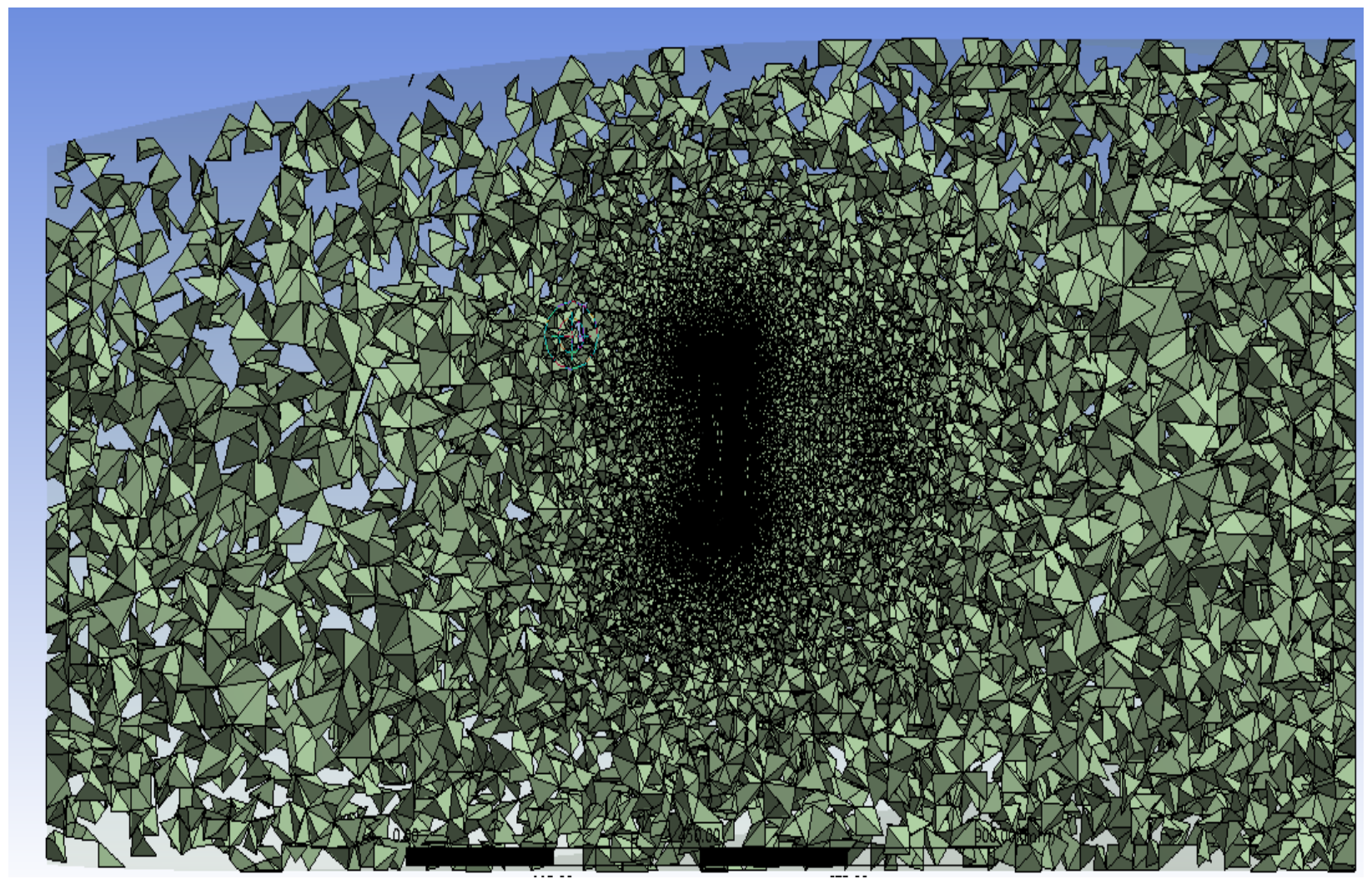

Figure 7 Distribution of Elements $\sigma$ Below Mean Quality

Conversely, these elements which are one standard deviation below the mean, and likewise roughly evenly distributed, though with perhaps a slightly more pronounced density upstream of the disc.

When it came to designing the shape of the control volume, initially a sphere was chosen to best represent the conditions of the experimental work. In this scenario, a large enough sphere would serve as a surrogate to ambient atmosphere. A key premise to Aliptera Aircraft's work is that the lip wing functions without an induced flow (i.e. when the freestream velocity is $\sim 0$ ), thus making the lip wing very useful for VTOL and STOL aircraft. Unfortunately, a large spherical 
control volume caused many of the same issues as the smaller spherical region, namely a great deal of poor quality elements. In addition, without an induced flow in the spherical control volume, reversed flow was a significant problem. [Stan]

The numerous elements experiencing reversed flow strongly inhibited convergence, especially for the continuity equation, which even with many thousands of iterations rarely dipped beyond $10^{-2}$ absolute convergence, even with only a first order solver, heavily relaxed. The only solution seemed to be inducing a small flow with a non-zero pressure boundary condition, but the size of the control volume's surface area meant that even a small amount of pressure translated to a very large force in absolute terms. Alternative attempts were made using a cylinder capped with half a sphere but with no real improvement in convergence unless flows were induced by pressure conditions.

The solution was to use a cylindrical control volume in the form of a digital wind tunnel, with pressure conditions at both ends. While this does not wholly eliminate the problem of induced flow, the necessary pressure to resolve the reversed flow issue could be measured in centipascals instead of or even kilopascals. This also means that the pressure at both ends would be three orders of magnitude less than the force of the actuator disc, so it is reasonable to assume that the flow effects will be much more dependent on the actuator disc than that of the boundary condition. 


\subsection{Geometry}

The angle of the lip with respect shroud was the primary area of interest in this investigation. Models were made with this in mind, using constraints in CATIA to allow the angle of the lip to vary while the length of the lip remains constant. Thus the surface area of the lip varies to form the shape of the lip.

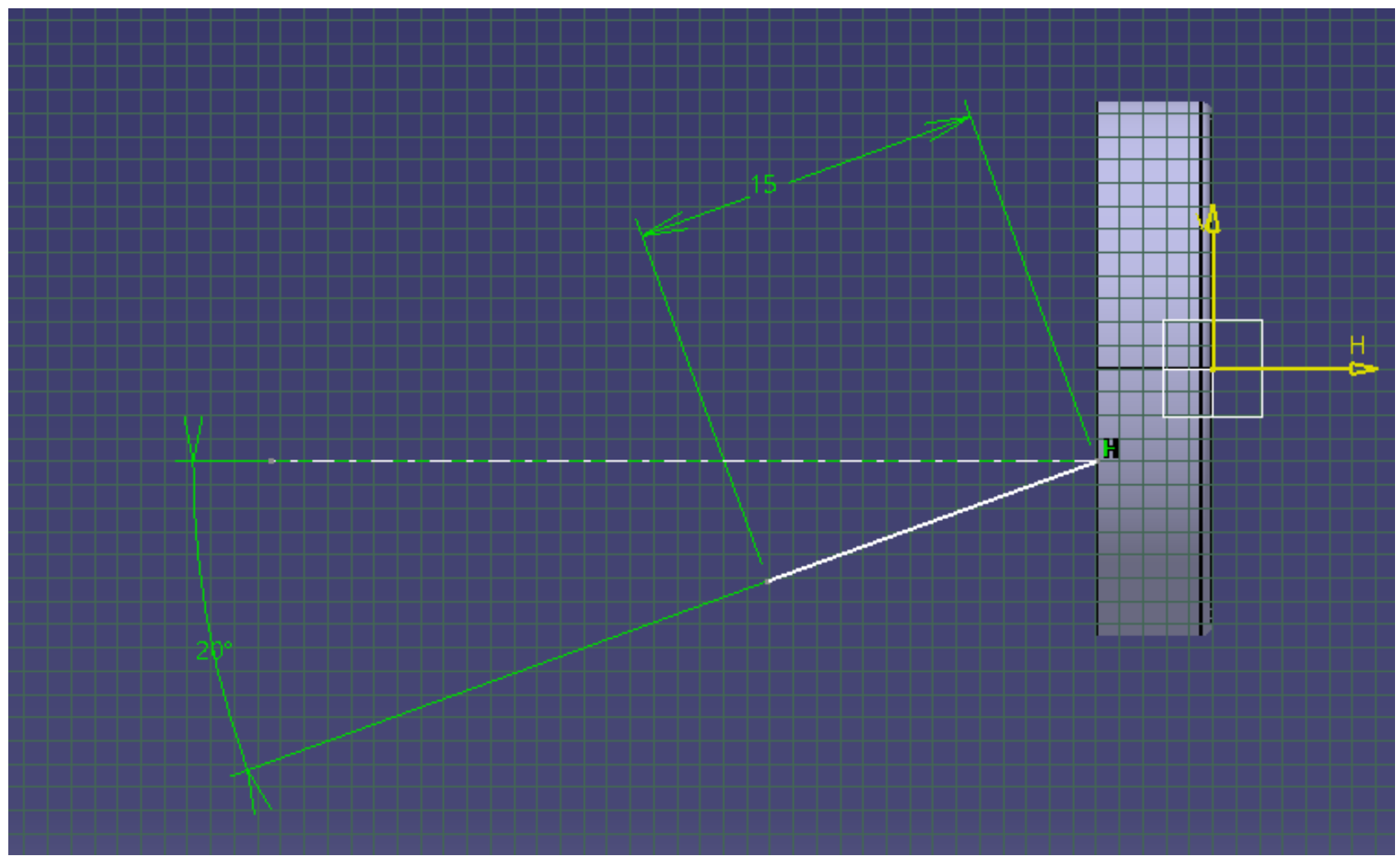

Figure 8 Shroud with Lip Geometry Constraints

Models were made for angles from $0^{\circ}$ to $60^{\circ}$ in $10^{\circ}$ increments. In all cases the angle of the lip is made from the mid-line of the shroud, highlighted in red in the figure below. 


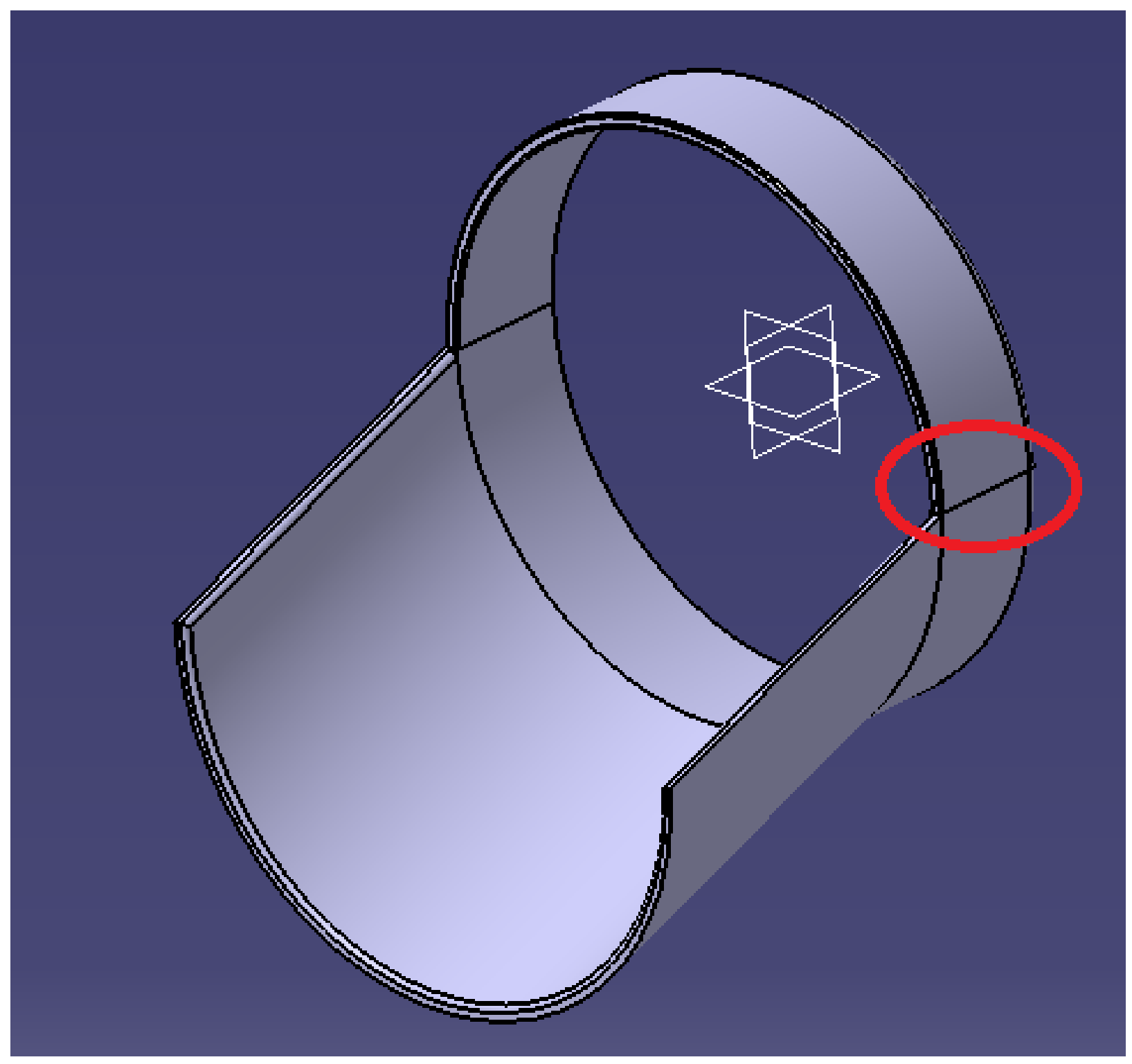

Figure 9 Lip Angle Definition

The sharp edges of the models proved a very serious meshing issue at first. Indeed, for $0^{\circ}$ and $10^{\circ}$ lip wings, the ANSYS Mesher was unable to form a grid that met the minimum quality requirements the software has as a safeguard against producing truly egregious models. In order to resolve this, an edge fillet was placed around all the sharp edges. While this curvature did require additional elements to be meshed successfully, it allowed the meshing to proceed. This fillet is a reasonable modification to the geometry as the radius is quite small (less than a tenth of 
a centimeter), and the original experimental setup was made of a soft, rounded foam like material, not specifically made for sharp edges at would be expected with harder polymers or metals. [Stan]

An isometric and side view of the $10^{\circ}$ angle lip model and its containing control volume and inner cylinder are shown below.

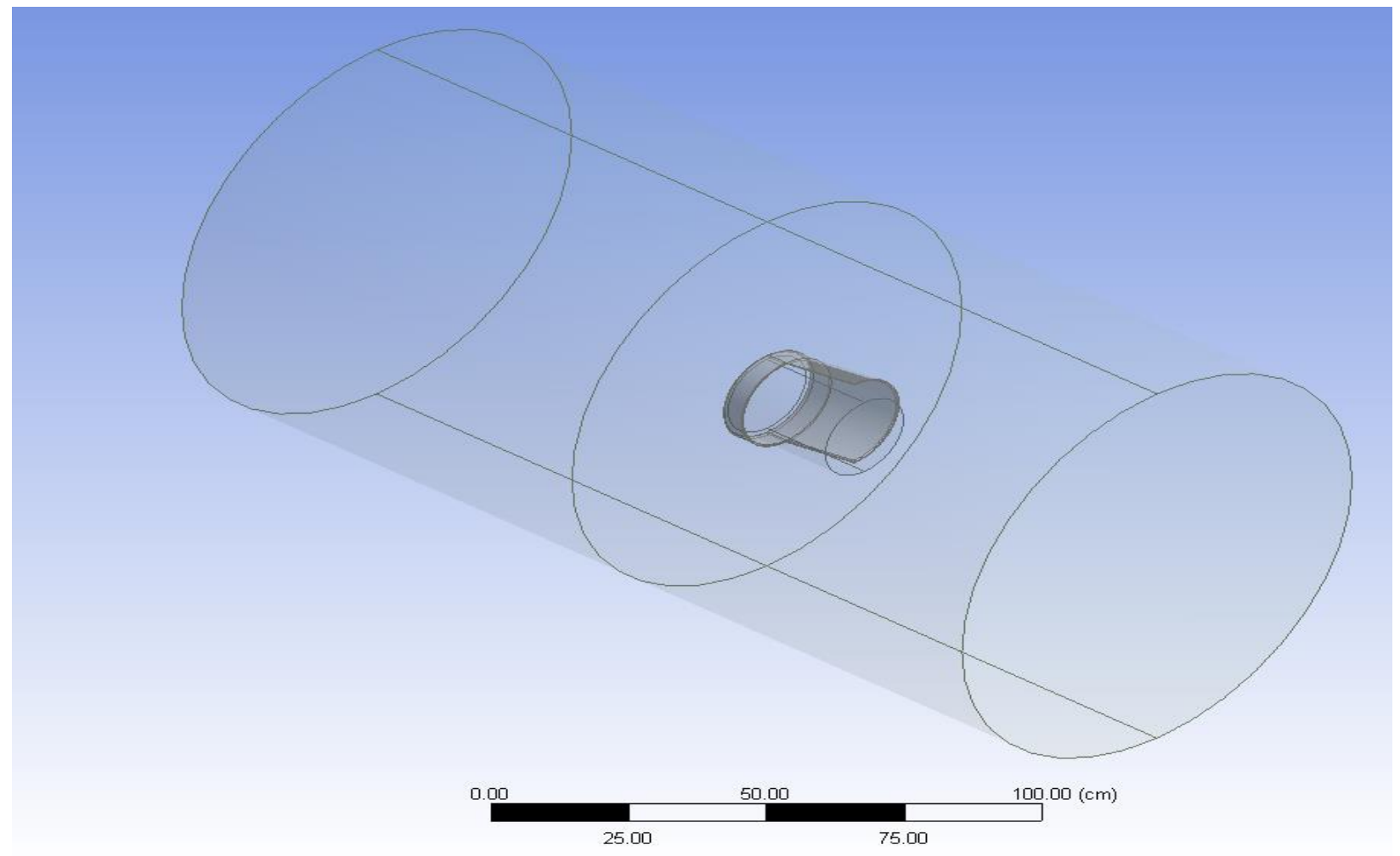

Figure 10 Isometric View of $10^{\circ}$ Lip Angle Final Geometry 


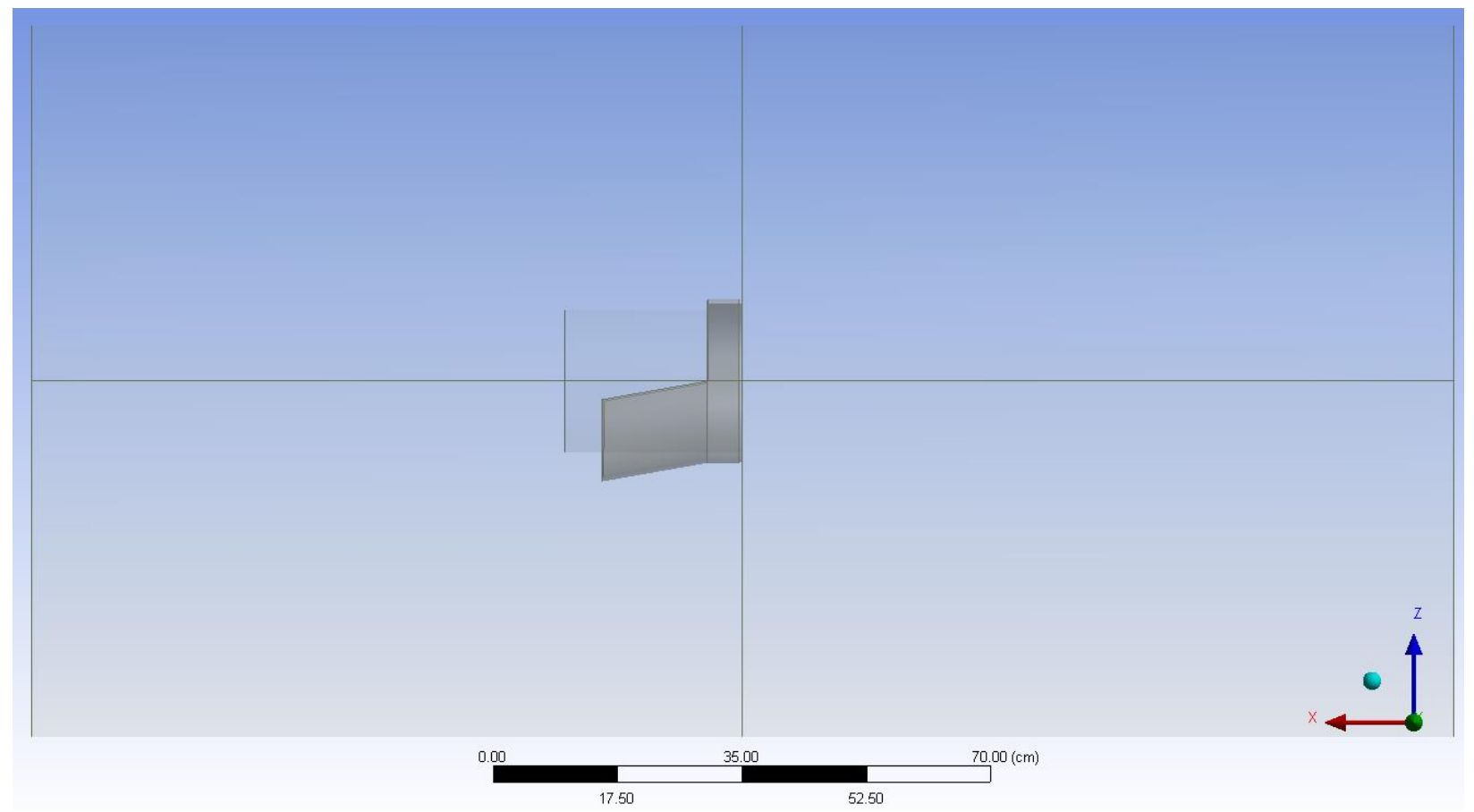

Figure 11 Side View of $10^{\circ}$ Lip Angle Final Geometry

The control volume was purposely designed to be large based on a review of the literature. It's important for the control volume to be sufficiently large for the flow to be fully developed both up and downstream of the actuator disc. This distance is generally defined in terms of the diameter of the actuator disc (D), and while no concrete rule exists, 5-7 diameters both up and downstream is found in several references.

\subsection{Grid Independence Study}

Grid independence, also known as mesh independence, is an important indicator of the reliability and accuracy of CFD solution. As mentioned previously, the majority of modern CFD 
solvers work on the principle of dividing a larger control volume into a series of smaller finite volumes (the so called finite volume method). There can be confidence in a CFD solution if it can be shown that the solution does not vary beyond some allowable limit the with the addition of more elements. That is, at some point, adding more elements will not improve the accuracy of the solution. [Cummings et al.]

Obviously, it is desirous to achieve grid impendence as quickly as possible. Every additional element is an additional albeit small computational load and when meshes can number in the hundreds of thousands if not millions, small individual loads can quickly aggregate to very large penalties indeed. This challenge is further highlighted by the fact that relatively small changes in mesh size can provide the illusion of grid independence. [Cummings et al.]

Consider for example $\sim 13,000$ hexagonal element mesh of a simple pipe as shown in the figure below. When the boundary condition at the pressure inlet is specified as a non-zero pressure, flow will be induced down the course of the pipe. 


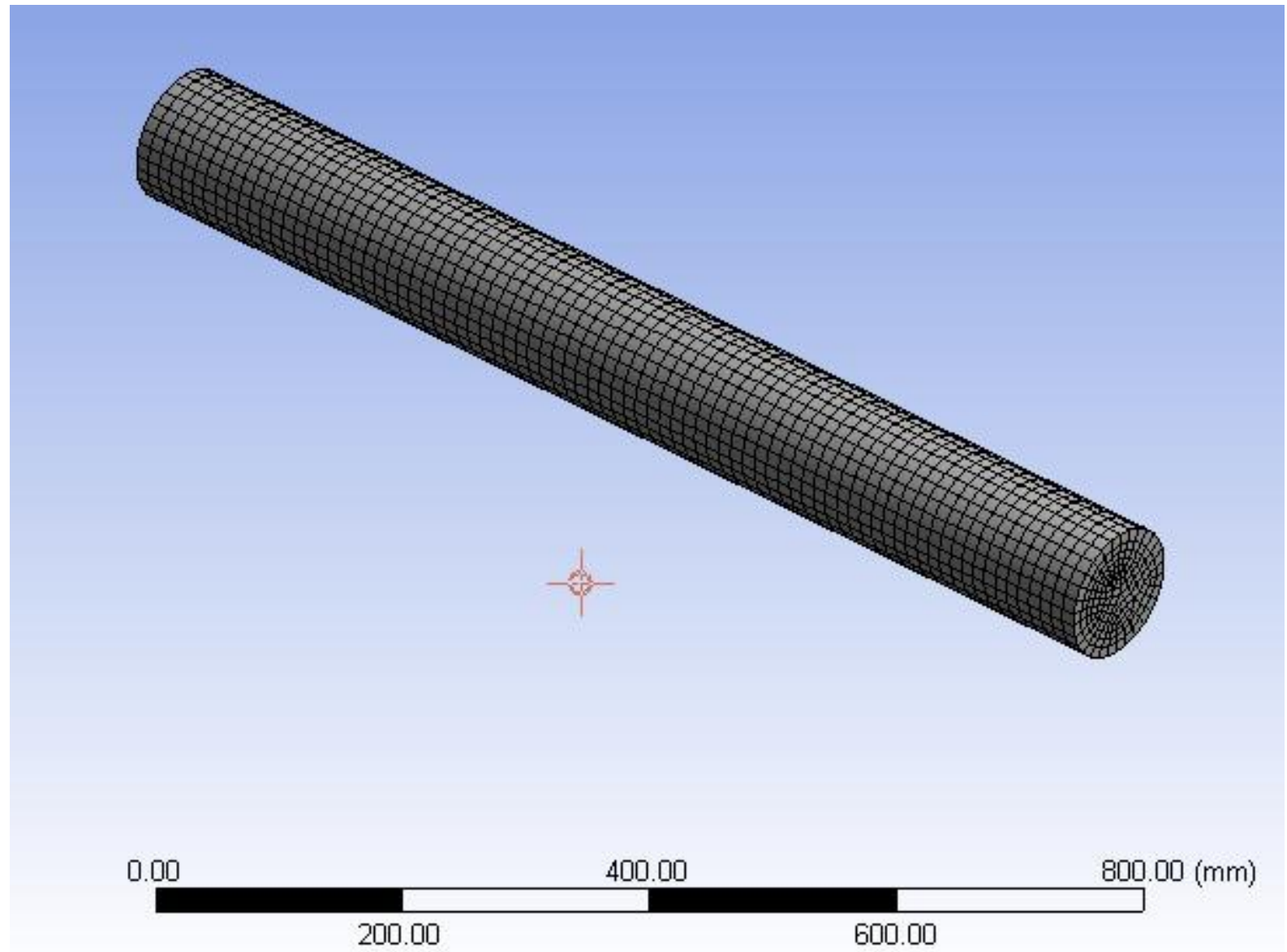

Figure 12 Pipe Meshing Example

The force balance for this particular pipe would be simply the input force, minus a loss term (due to viscosity / skin friction with the pipe wall) being equal to the output force, as shown in the equation below.

$$
F_{o}=F_{i}-c_{f}
$$

Equation 6: Force Equation of a Pipe with Skin Friction 
The output force should be independent of the grid size, and so it can be said that grid independence occurred when $F_{o}$ no longer varies with the addition of more elements beyond a certain level of significance. Increasing the mesh on this pipe by 1000 additional elements, or less than $10 \%$ of the original mesh size, will produce a change in of $F_{o}$ only $3 \%$. One might conclude from this that mesh independence has been achieved, but by doubling the size of the mesh, $F_{o}$ changes by $15 \%$ which is proof that the model is varying with respect to the grid size. Thus to prove that mesh independence has been achieved, it is often necessary to use fairly large step sizes.

To facilitate the grid independence study for the lip wing models, the control volume was set up with only the actuator disc alone (as shown in the figure below) and then the thrust generated by the disc alone was calculated for each mesh size. 


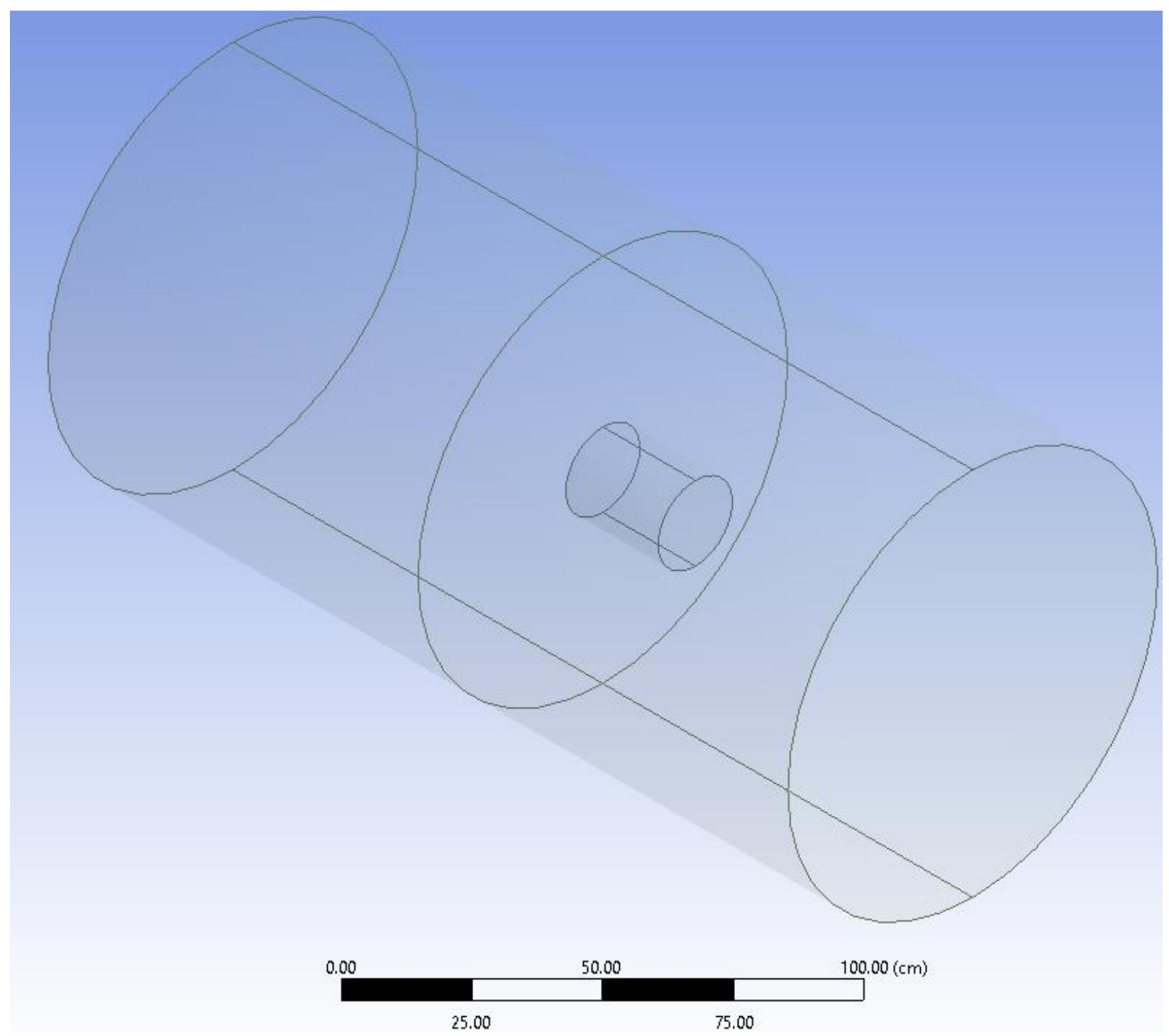

Figure 13 Actuator Disc and Control Volume Geometry

In order to determine if the order of accuracy will make a major impact on the solution, each model is solved at both first and second order solvers. It was determined early on that for some reason the first order solution was not sufficiently accurate 
When plotted, the normalized thrust vs grid size form a quasi-logarithmic pattern for both first and second order solutions. The major changes in thrust taper off when the number of elements approaches 1 million, and mesh independence can be said to have occurred at between 2-3 million elements for the second order solution, as it begins to vary only $3-4 \%$ and at 6.2 million elements, the difference from the ideal thrust is only $2.4 \%$.

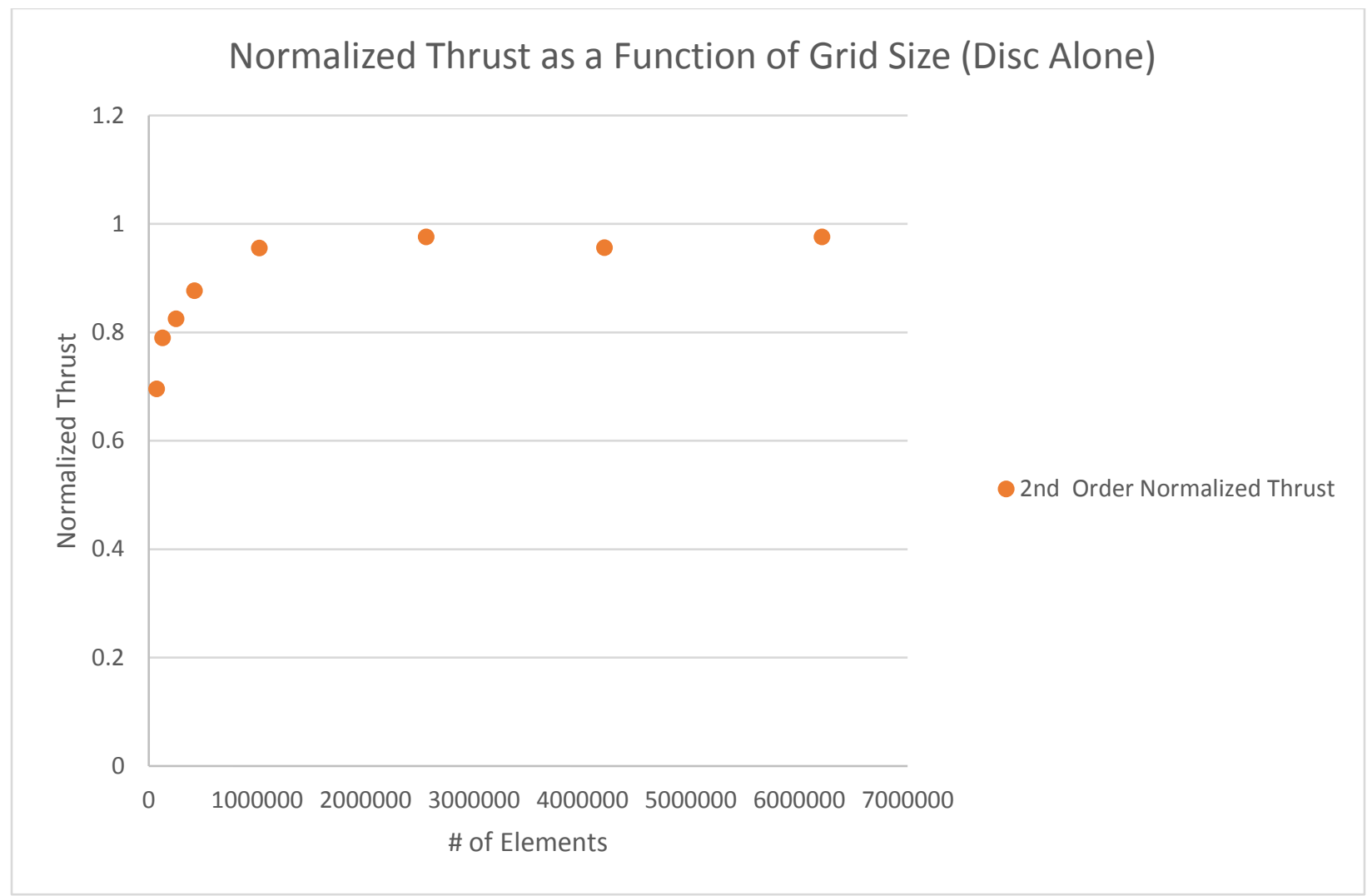

Figure 14 Normalized Thrust of Actuator Disc Alone as Function of Grid Size

The lower than predicted thrust makes sense in the context of numerical diffusion in the flow. If this is in fact the case, then there should be evidence of momentum diffusion in the flow, which would appear as the formation of a boundary layer or shear between fluid zones, despite the fluid being explicitly declared inviscid, only in this case the momentum diffusion is not the 
result of viscous forces but numerical error. When looking at a cross section of the velocity contours, we see this is in fact the case.

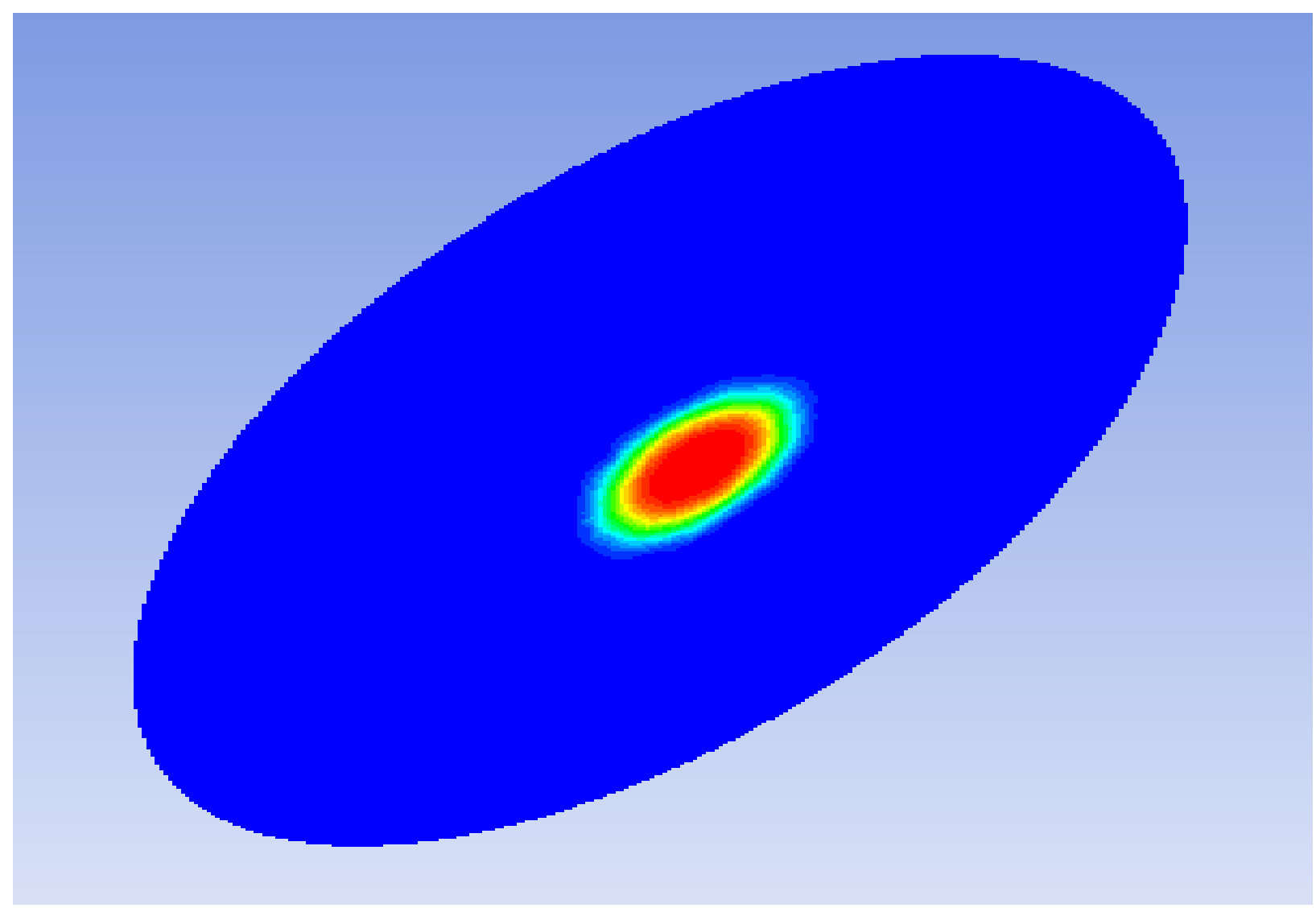

Figure 15 Example of Numerical Diffusion at Outlet for 1st Order Solution

\subsection{Final Meshing}


The final mesh was primarily dominated by tetrahedral elements, with some hexagonal, pyramidal and wed-6 elements. For models with a greater lip wing angle, for example when $\alpha$ is $40^{\circ}$, the Tet elements strongly dominated as shown in the graph below.

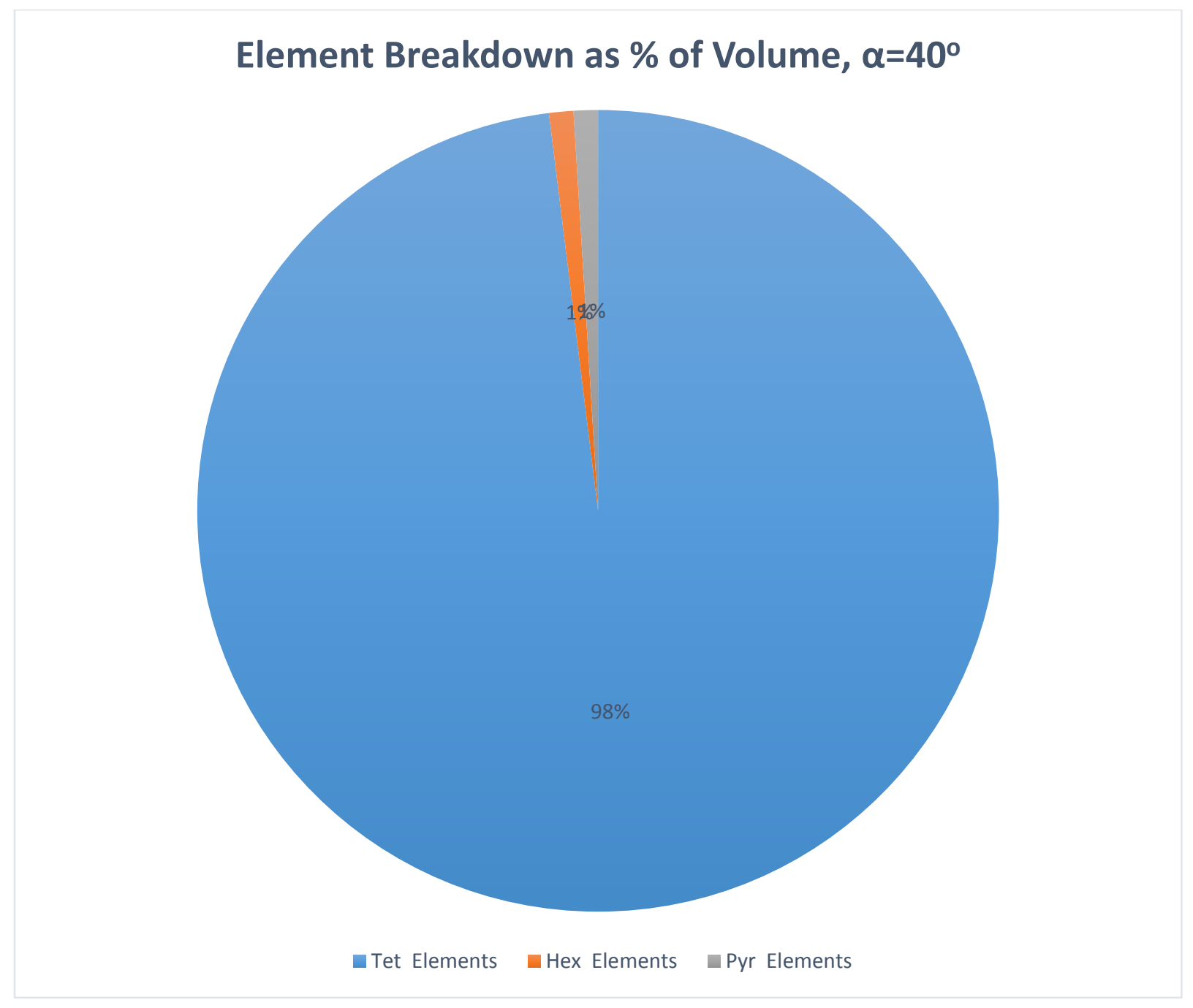

Figure 16 Element Breakdown of Mesh, /

However, for models with lower values of $\alpha$ such as $0^{\circ}$ or $10^{\circ}$, the dominance the Tet elements was less pronounced. The remaining element types almost double in magnitude, although in absolute terms they are still quite infrequent compared to Tet elements. 


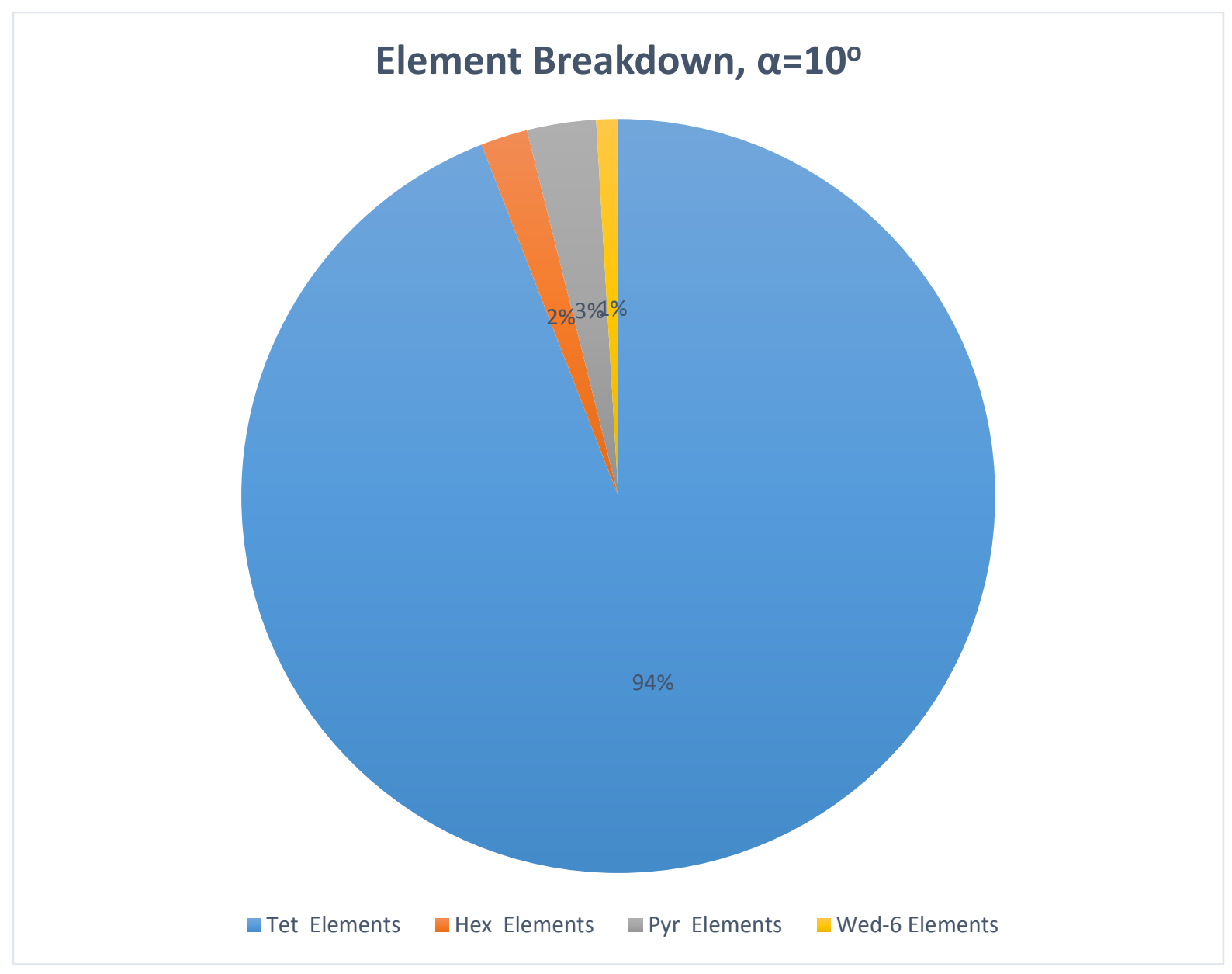

Figure 17 Element Breakdown of Mesh, $\alpha=10^{\circ}$

Overall element quality was quite high, with a consistently high average element quality being achieved for all models. For all models it ranged from 0.827 to 0.842 , averaging at 0.831 with a statistical spread of only $1.8 \%$. While the average mesh quality did vary somewhat it is difficult to infer any patterns from the variations, shown in the below figure. 


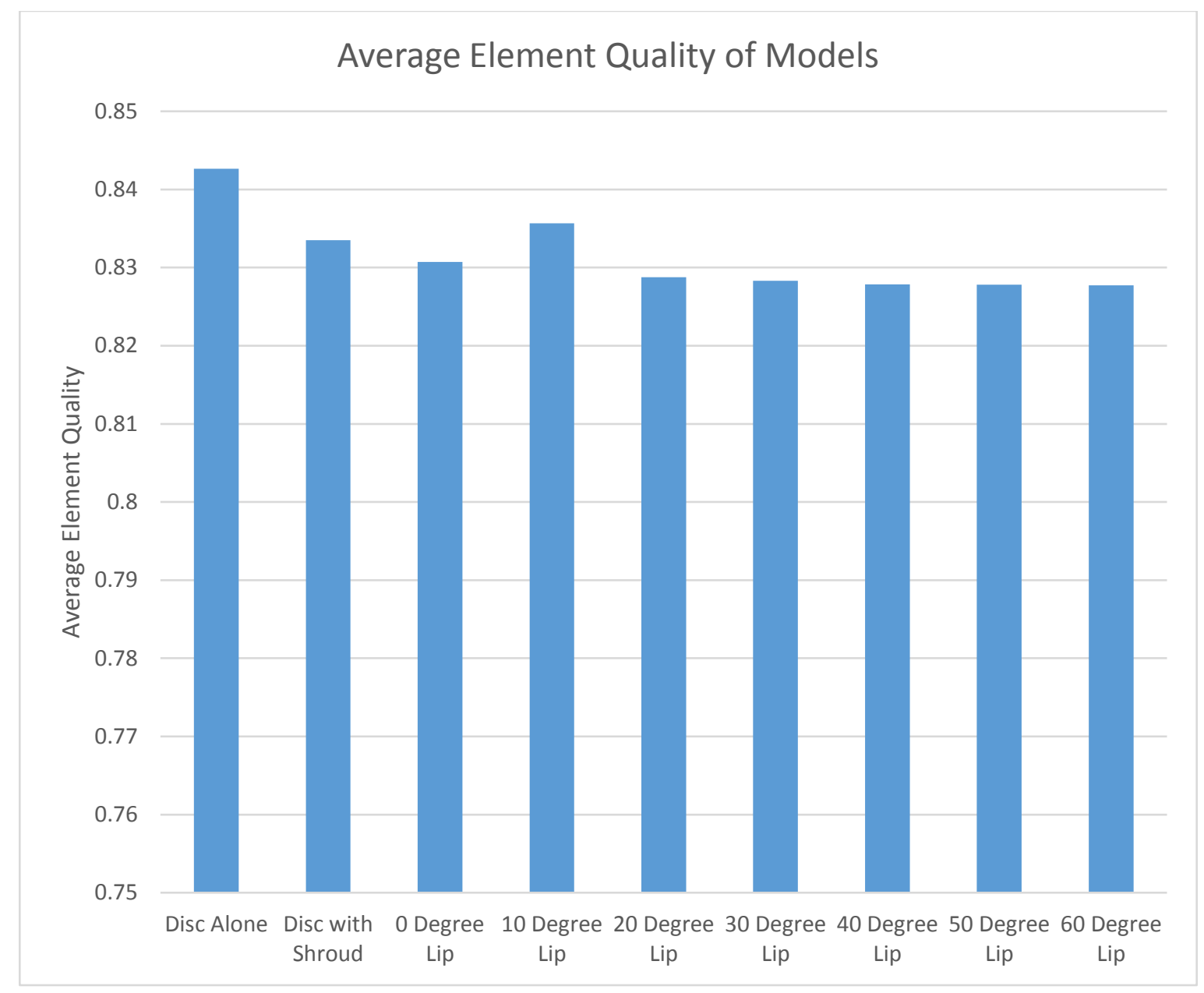

Figure 18 Average Element Quality of Models

While some conclusions can be drawn, for example the addition of the lip and shroud geometry to the disc and control volume alone results in a slightly lower quality mesh. However there appears no definitive relationship between the value of $\alpha$ and the average quality of the mesh. The figure below shows an X-Y scatter plot of $\alpha$ versus average element quality. Though there is a spike around $10^{\circ}$, it's only a variance of 0.03 . With such a small variance, it is difficult to draw any conclusions, at least from an instinctual perspective. 


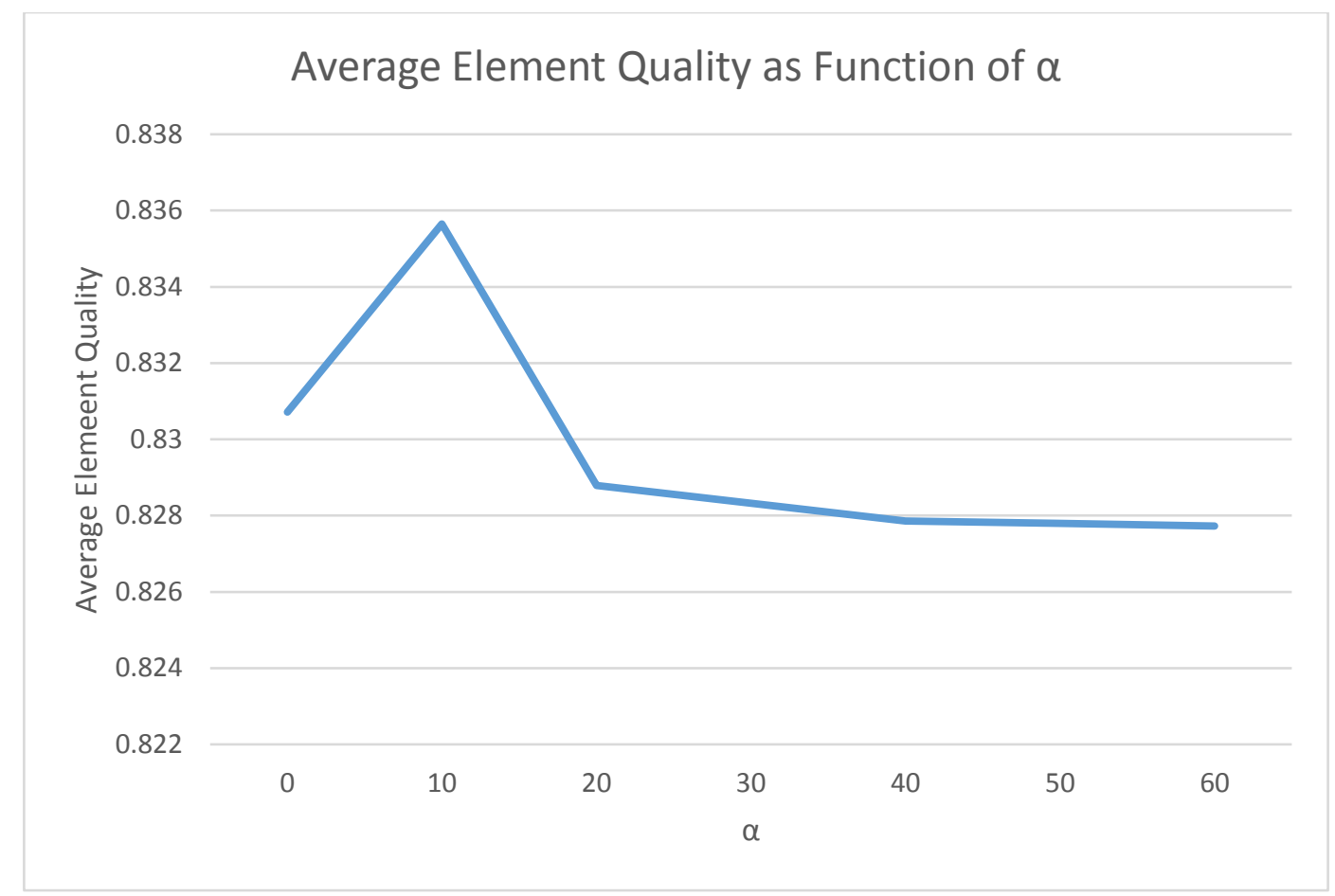

Figure 19 Average Element Quality as Function of Lip Wing Angle

There's also arguably a decreasing trend from $20^{\circ}$ to $60^{\circ}$, but this variance is only 0.01 , and so even less definitive. The conclusion that can be taken away from this is that it is unlikely that there is any significant variation between the meshing of the models, and relatively equal confidence can be had in the results from each model.

In general, the mean is significantly above what would be expected from a normal positive generation, and tapers off significantly to left of the mean. 


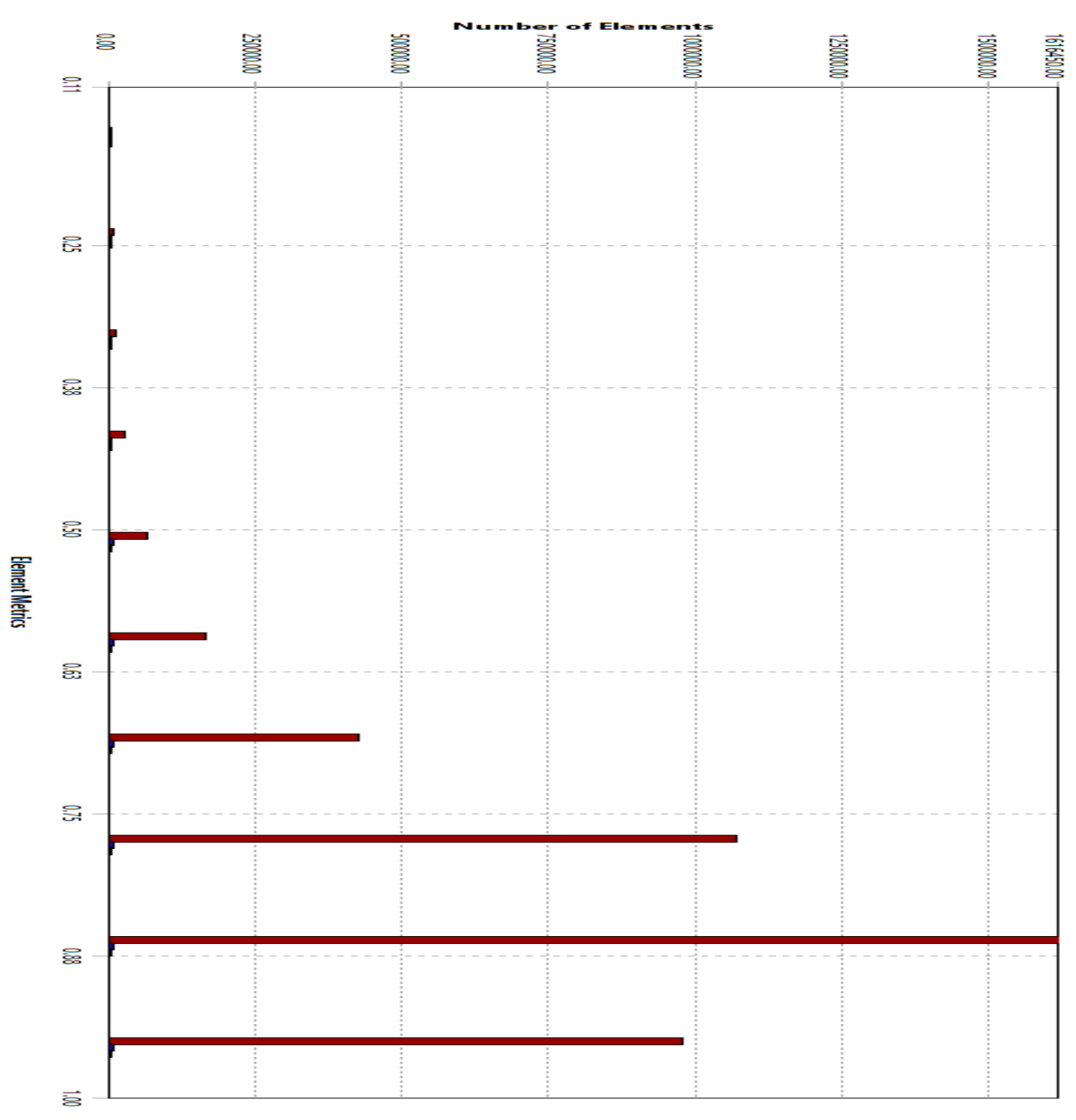

Figure 20 Distribution of Elements By Element Quality

\subsection{Boundary Conditions}

Apart from the inlet and outlet, which are both assigned as simple pressure boundaries, there is one more boundary condition that is necessary. The fan boundary condition works to create the actuator disc. It can be defined in a variety of ways, as a merely a pressure 
discontinuity, or as a velocity discontinuity. It is also possible to impart swirl to the flow as it passes through the boundary. It is even possible to define the actuator disc not as a constant change in pressure or velocity, but as a gradient radiating outward.

For simplicity's sake, the fan boundary condition was set as simply a pressure discontinuity, much as classical actuator disc theory would envision it. The thrust was calculated based on the experimental work by Stan. Based on the change in momentum observed, it was possible to calculate the force, and from there using actuator disc theory it is a trivial matter to calculate the necessary pressure across a given area to achieve the desired force. 
The calculation is shown below:

$$
P=\frac{F}{A}=\frac{0.19635 N}{\pi\left(\frac{10 \mathrm{~cm}}{2}\right)^{2}} \approx 25 P a
$$

\subsection{Solution Data Collection}

FLUENT allows the user to export solution data in a variety of ways, by creating rakes, lines through control volume or just exporting the solution data from an entire surface. For simplicity's sake, the solution data at the inlet and outlet of the control volume was exported into Matlab $^{\mathrm{TM}}$ for analysis. Specifically, the density, velocities and face areas of each element at the inlet and outlet were exported. Referring back to Equation 3, it is clear the change in thrust can be calculated from the summation of the momentum in each element and subtracting the difference of the summations from the inlet to the outlet.

This approach has several advantages. It allows for analysis of the control volume as a whole, rather than focusing on one specific area. It captures the effect of the actuator disc on the lip wing, rather than the reverse. Since the actuator disc is a fixed boundary condition this would seem to be a trivial solution. The premise of this analysis is that the propeller (represented in this model by the actuator disc) is creating an unbalanced resultant force on the lip wing. This resultant force can be resolved into two components, lift and thrust. Based on coordinate system displayed by Figure 11, the thrust force is defined as the x-direction component. Thus the equation for total thrust in the control volume is: 


$$
T=T_{\text {propeller }}+T_{\text {lip wing }}=\sum_{\text {outlet }} \dot{m} V_{x}-\sum_{\text {inlet }} \dot{m} V_{x}
$$

Equation 7 Total Thrust In Control Volume 


\section{Chapter 4.0 Results}

When looking at the results in the change of thrust versus the lip angle, it can be seen that that there is a general increase up to $30^{\circ}$, and with a drop off after the peak. The peak value is $\sim 12 \%$ above the thrust, a significant increase in thrust over the original actuator disc which tends to support the experimental claims made by Aliptera Aircraft that the lip wing has an impact on thrust.

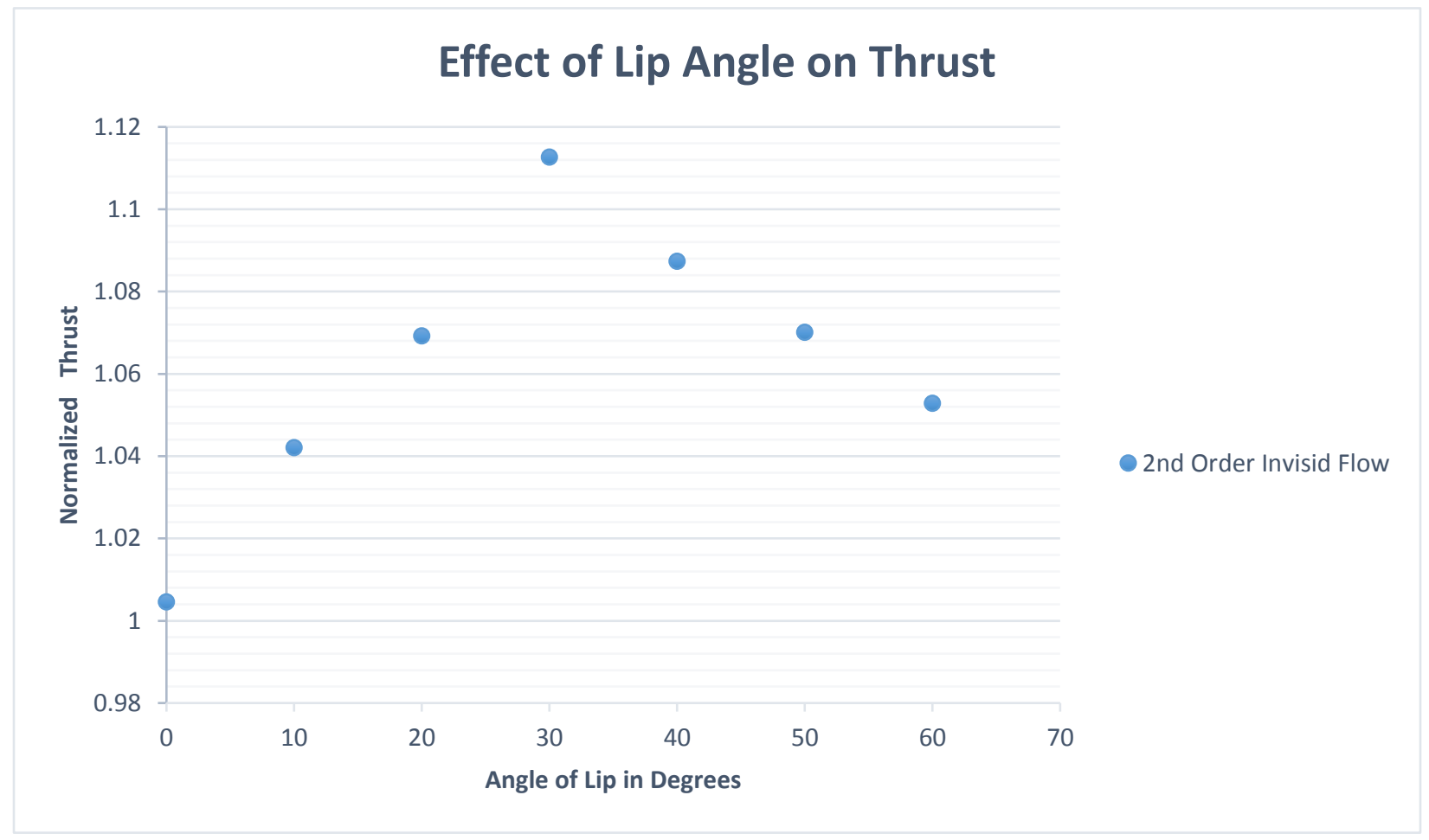

Figure 21 Effect of Lip Angle on Thrust

However, there is an anomaly that should be obviously apparent and needs to be addressed. There is a slight increase in thrust (0.04) when the lip wing is at $0^{\circ}$. It's difficult to understand how this could occur, as it seems to be contrary to the common understanding in fluid 
mechanics. A symmetrical lip shouldn't affect the flow in any measurable way. The scenario is akin to a symmetrical airfoil at $0^{\circ}$ angle of attack. The forces on both sides should remain balanced. It would be tempting to dismiss this increase in thrust as result of numerical error, as it is only $0.04 \%$ of an increase it is prudent to explore it more deeply before reaching that conclusion. Numerical error should not be used as a catch-all phrase for anything that cannot be immediately explained, therefore a careful analysis of alternative explanations should be undertaken before accepting numerical error as a conclusion.

The lip wing does not exist in isolation; it is part of a larger structure that includes a nominal shroud. This was done to better capture the original experimental work done by Aliptera Aircraft for the purpose of comparison. This raises the question, would the shroud by itself cause an increase in thrust? Could this explain the change in thrust seen with the lip at $0^{\circ}$ angle of attack?

Shrouds are frequently used in conjuction with propellers to increase thrust as with the experimental Bell X-22 aircraft. Putting a shroud (also called a duct) around a propeller signficiantly reduces the loss from tip vorticies at higher RPMs as well as (through appropriate sizing) increasing the velocity of air flow through the propeller.

Because this model uses an actuator disc, there are no blades and hence no blade tips to induce loses from. In addition, tip lose is generally modelled as a dynamic, time variant phenomenon, heavily influenced by viscous and turbulent effects. This model is a static, steady 
state, and inviscid. Therefore, tip loss and equivalent effects should not be present in this model. However, the shroud may be able to increase thrust in some other method, for example by altering the capture area of the fan or decreasing the inlet velocity to the actuator disc or by some other unknown means.

One way to answer the question of whether this small increase is caused by the shroud alone or is merely the result of numerical error, is to consider the shroud independent of the lipwing. To illustrate this, a mesh independence study was performed with the disc and shroud in conjunction, the results of which were normalized against the calculated thrust of the propeller. Due to the fact it had already been determined that a second order solution was required for sufficient accuracy, this study was done only using a second order scheme. 


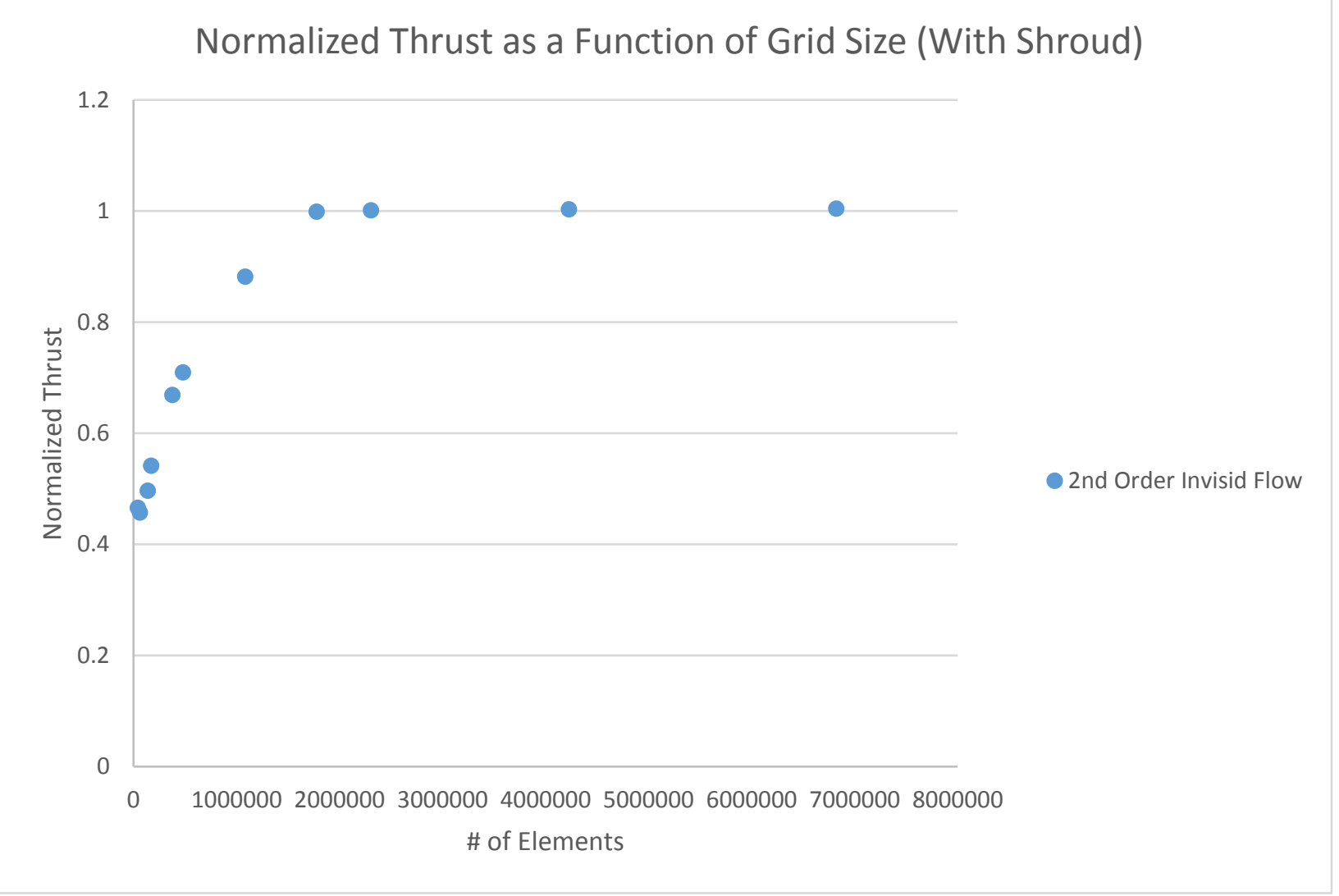

Figure 22 Normalized Thrust as a Function of Grid Size With Shroud Correction

As the above results show, the resultant thrust with a shroud behaves in a similar manner vis a vis the grid size as it did with the disc alone. A very sharp, linear improvement in accuracy occurs that tapers off around 1-2 million elements. However, with the shroud, the normalized thrust is slightly above the calculated ideal thrust, at 1.0036, or almost the same level of improvement that is seen with the lip wing at a $0^{\circ}$ angle. In fact, the increase in thrust with the shroud accounts for $99.89 \%$ of the improvement seen with the lip wing at the $0^{\circ}$ angle. When comparing the change in thrust of the shroud to that of the lip wing at a $0^{\circ}$ angle, the difference in normalized thrusts is only 0.001 . Such a small change in thrust is much easier to dismiss as simple numerical error, which may or may not be relevant, whatever the source might be. 
In order to isolate this issue from further analysis, or at least greatly reduce its relevance, a simple solution was devised. Rather than normalizing the thrust of lip wing cases again those of the disc alone, the thrust was normalized against the grid independent case of the disc and shroud together. By normalizing the thrust in this manner, any resultant effect is unarguably that of the lip wing and its angle, not that of the shroud. Looking at this second graph, shown below, the normalized thrust when the lip angle is $0^{\circ}$ is approximately at unity. Instinctually, this makes a great deal more sense. 


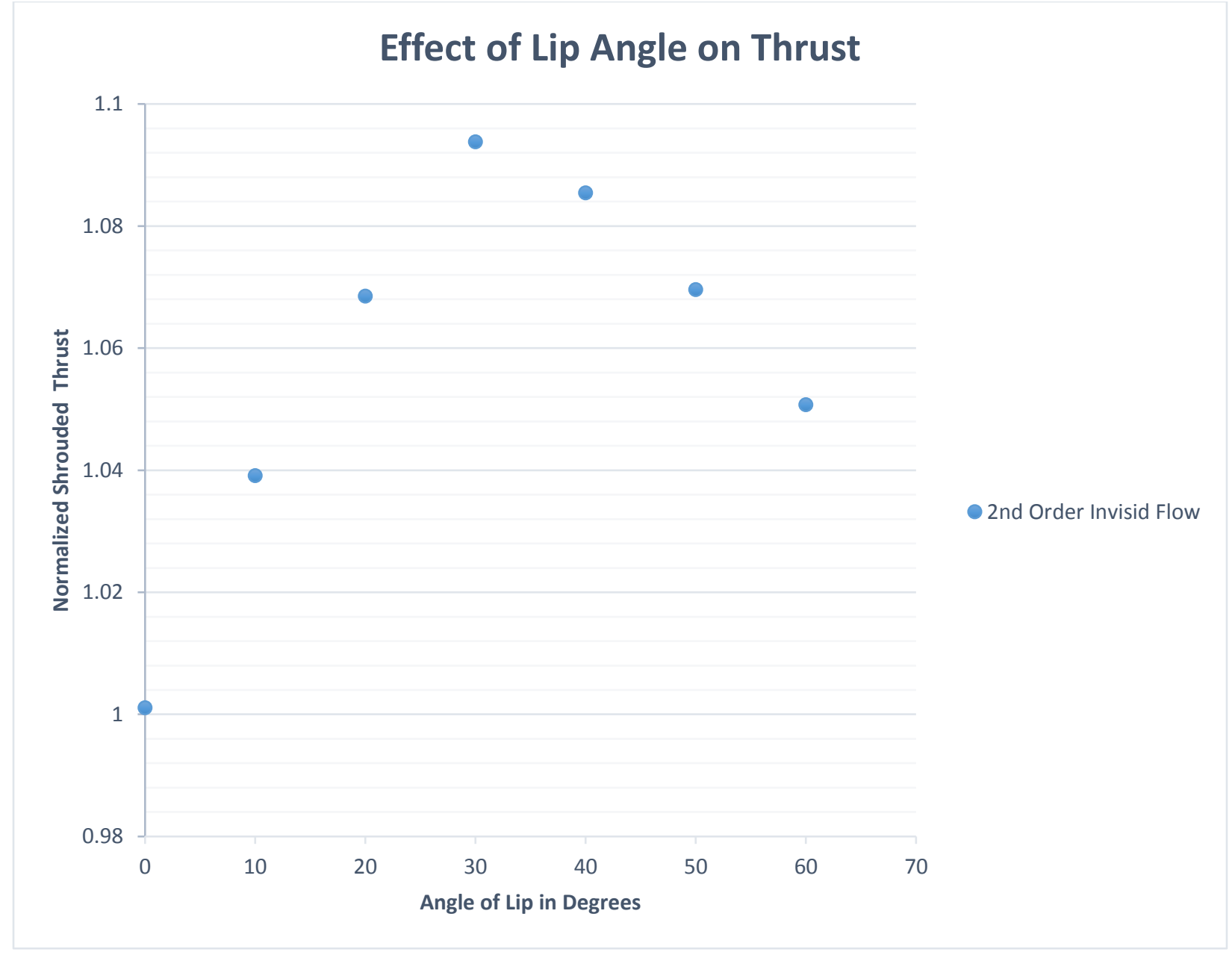

Figure 23 Effect of Lip Angle on Thrust (Shroud Normalized)

Even when taking into account the effect of the shroud, the basic pattern seen before remains very similar. Thrust increases with lip wing angle up to $\sim 30^{\circ}$, before tapering off. However even at $60^{\circ}$, or twice the approximately optimum angle there is still a pronounced increase in thrust above the baseline shrouded thrust, assuming numerical error is not significant. 
In order to allay concerns about numerical error it is necessary to turn the discussion to that direction. Numerical error is an issue that all models must address at some point or another. It is in a sense, analogous to the experimental error encountered when doing physical tests: namely it is the gap between the equipment and the natural world. Numerical error can take many form, one of which has already been discussed, truncation error. Truncation error stems from the fact that a Taylor series expansion or something similar, must eventually end, i.e. be truncated. A $\mathrm{n}^{\text {th }}$ order Taylor series will always have higher truncation error than an $\mathrm{n}^{\text {th }}+1$ term. In an ideal world one would set $\mathrm{n}$ as close to infinity as possible, and indeed, in some very special but fortunate cases "sufficiently close to infinity" can be achieved with very small numbers in absolute terms.

In basic terms it is the same in computational fluid dynamics. The second order solution scheme will reduce truncation error compared to the first order scheme, but of course as previously discussed this is at the cost of greater numerical stiffness and computational loading. While Ansys Fluent does support third order schemes, these often come at a significant penalty towards stability, especially for unstructured grids. For this reason they tend to only be pursued for more complex models; for example models involving turbulent flows where the issue of false diffusion caused by a lower order scheme would be a serious hindrance to the investigation. In general, a second order upwind scheme is considered sufficiently accurate for a simpler, inviscid model. This is supported by the previously demonstrated fact that for a sufficiently large mesh, the error between modelled thrust and he calculated thrust was negligible. [ANSYS Inc] 
Another important aspect in quantifying the allowable confidence one can have in a CFD solution is the degree of convergence displayed by the residuals of the iterative solution. When dealing with the absolute convergence criterion, such as the one used in this analysis, residuals can be thought of as the error between two iterations. As the number of iterations increases, it is hoped that the residual of the various equations will decrease. In practice this behavior may not be a simple relationship. If convergence is occurring, it may be a linear or ideally an exponential decay function between iterations and residuals. Also possible is a pattern more reminiscent of a dampening effect.

A dampened harmonic pattern can sometimes be observed. In general this kind of convergence behavior would not be seen as an invalidating negative. The general trend of the residuals is still towards 0 , even though there is still some fluctuation. Ideally however, it would be preferable for the oscillations to decrease in amplitude as the iterations increase.

Other convergence behavior that may at first appear to be concerning or would raise doubt about the results are long periods of minor to no improvement, or even a somewhat retrograde performance (i.e an increasing residual). While this is certainly an annoyance, as the figure above shows it is not necessarily a critical failure. A short period of retrograde behavior, or even a long period is not necessarily a sign that a solution is of poor quality, or even that a high quality solution cannot be achieved. As shown in the above figure, after enough computational time, the residuals can again begin to converge. 
Convergence is not guaranteed however, and its occurrence can be surprisingly deceptive. Much as randomness can very difficult to prove, it can be equally challenging to prove that convergence has been achieved to within a degree of reasonable doubt. Serious oscillation can sometimes occur in residual functions. It's entirely possible that at the low point of an oscillation period to lay below the convergence limit that may be required for a given modelling exercise. However, it is hard to argue that this is true convergence when adding perhaps a small number of iterations will lead to a massive increase in residual error. This example is obviously taken to the extreme but it is also possible that the period of oscillation could be dozens or even a hundred of iteration long. In such a case, one might well have the illusion of greater convergence and an unfounded confidence in the model's results than is actually warranted.

When looking at the models for the different lip angles, convergence behavior tended to be fairly typical. A rapid decline in residual was followed by a quasi-plateau of slower decline. The following figures are the preliminary residuals of continuity, $\mathrm{x}, \mathrm{y}$ and $\mathrm{z}$ velocities for the $20^{\circ}$, $40^{\circ}$ and $60^{\circ}$ lip wing models. 


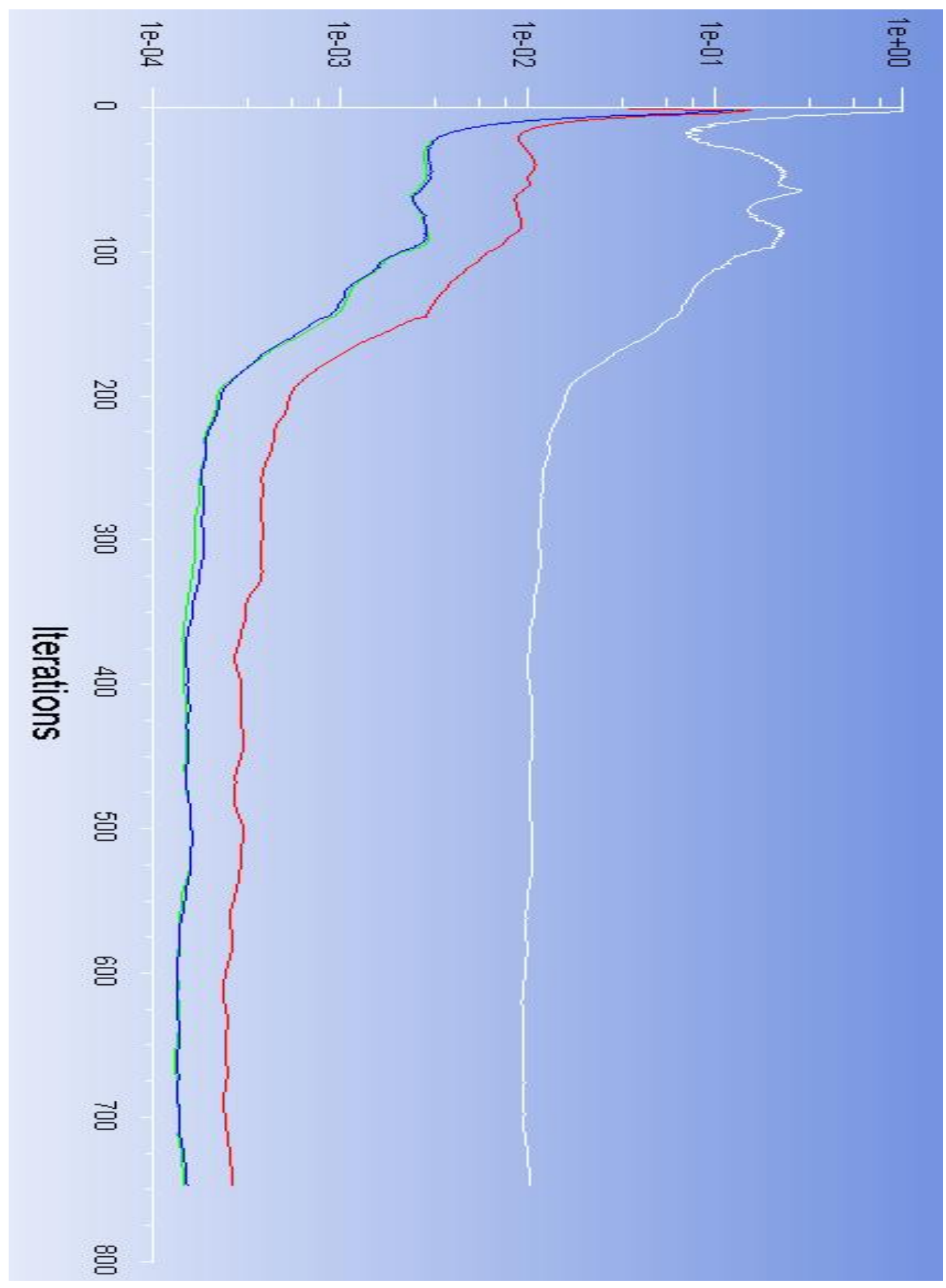

Figure 24 Residual Behavior for $\alpha=20^{\circ}$ 


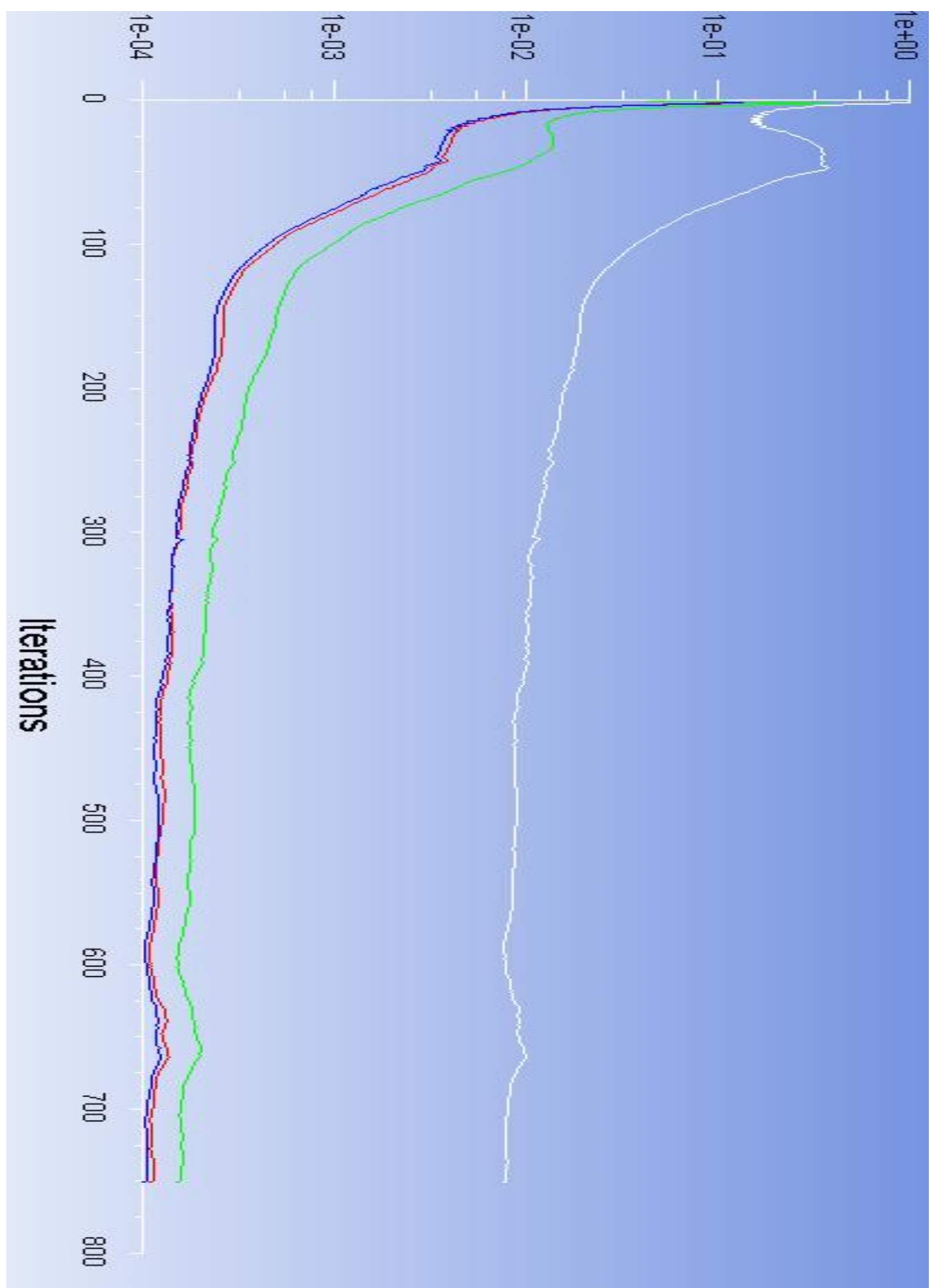

Figure 25 Residual Behavior for $\alpha=40^{\circ}$ 


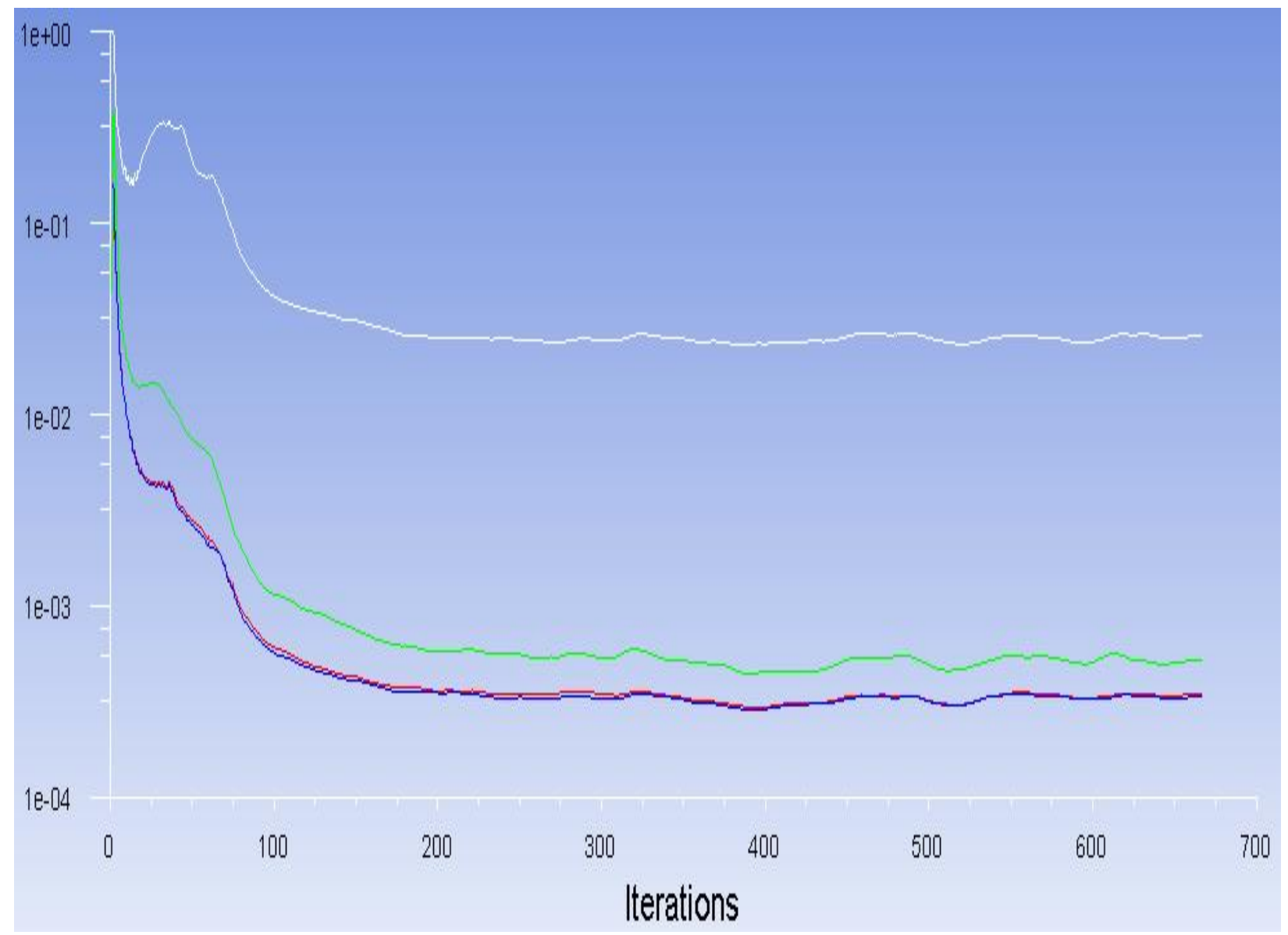

Figure 26 Residual Behavior for $\alpha=60^{\circ}$

These were all taken in a similar iteration bandwidth, (from 650-750). All show similar behavior, a sharp initial drop that tappers off at around approximately 200 iterations. There are slight fluctuations beyond the $200^{\text {th }}$ iteration but not much progress is made otherwise towards convergence. Increasing the stiffness of the equations did not noticeably improve convergence behavior unless taken to extremes where it induced a sinusoidal pattern of oscillation. After 
almost 2000 iterations, there was a slow but steady trend towards convergence, however it was nowhere near the level of convergence that would be required for confidence in this model.

The solution to this problem was to slightly increase the mesh density with a refinement around the fan boundary condition, as well as going with a SIMPLEC solver instead of the original SIMPLE solver. SIMPLE solvers are generally considered sufficient for simpler models using inviscid or laminar flow. However, the SIMPLEC generally results in faster convergence, as much as $20 \%-30 \%$ than the SIMPLE algorithm, while not unduly adding to the computational load as the SIMPLER algorithm.

The figure on the next page shows the final residual behavior of the lip wing at $30^{\circ}$, chosen as it is the lip wing angle that shows the highest level of thrust increase. It shows clearly that with a SIMPLEC solver the convergence behavior is much more pronounced, allowing for a more reasonable level of convergence that can inspire more confidence in this analysis. 
However, this leaves a question, what is an acceptable level of convergence? This is of course somewhat of a loaded question, one that does not have an easy, concrete answer. It depends greatly on the system that is being modelled, and is limited by the variables under consideration. For example, the energy equation, especially in the second order form, can display extreme numerical stiffness. An absolute convergence of $10^{-5}$ is considered fair to good for most simpler cases while a convergence level of $10^{-7}$ is often seen as excellent.

Ideally, numerical results of various levels of convergence could simply be compared to experimental values, for example from a wind tunnel. However, to do such experiments would defeat many of the advantages of CFD, such as the ability to rapidly analyze different designs and conditions and lower fiscal cost. A more practical method is to examine the solution for certain concrete metrics which can support or refute the confidence one would have in the model.

This is of course a somewhat roundabout, indirect method. Like Unfortunately, this is sometimes the limit of our ability to analyze flows. In many CFD codes it is a trivial task to display contour maps of static and dynamic pressure, or even metrics such as turbulent energy dissipation or molecular viscosity. In physical experiments it is not so simple.

When considering a CFD model and what metrics can be used to evaluate it, it's important to keep in mind what is being evaluated. As an example, consider a CFD model of a 
nuclear fuel rod in a storage tank. If the model's primary purpose is to model the temperature of the fluid in the storage tank, then the level of convergence of the energy equation is of primary concern. How then to determine if the level of convergence necessary for acceptable modelling? In this case, what can be determined in the code is the amount of energy in the control volume (the storage tank) and how much energy leaves. From the first law of thermodynamics, this amount of energy should be equal to that of the energy produced by radioactive decay in the fuel rod. This energy production would be defined in the model, and therefore can serve as the benchmark of a percentage error calculation.

Thus, if for example at an absolute convergence criteria of $10^{-3}, 97.4 \%$ of the energy is accounted for, one can have a reasonable discussion of whether that's an acceptable level of convergence. If one is doing a preliminary design study, then $+/-2.6 \%$ might be sufficient for those purposes, or it might be justification for using a more computationally expensive solver such as the SIMPLER algorithm.

For the lip wing models, the key metric being investigated is thrust. For the case of actuator disc working alone, it was possible to compare the thrust produced to the thrust specified by the fan boundary condition. Obviously this is no longer possible when the lip wing is introduced but what can be analyzed objectively is the change in mass flux over the control volume. As nothing involving exotic physics is being modelled, the change in mass flux from inlet to outlet should be zero. Due to numerical error this is not going to be the case, but this 
allows for the discussion of reasonable levels of convergence from the standpoint of concrete numerical metrics rather than an instinctual view or heuristic best practices.

The results of the change of mass flow rate between inlet and outet at different lip wing angles, at a convergence level of $10^{-4}$ for the continuity equation, and a convergence level of $10^{-5}$ for the $\mathrm{X}, \mathrm{Y}$ and $\mathrm{Z}$ velocity equations are presented below.

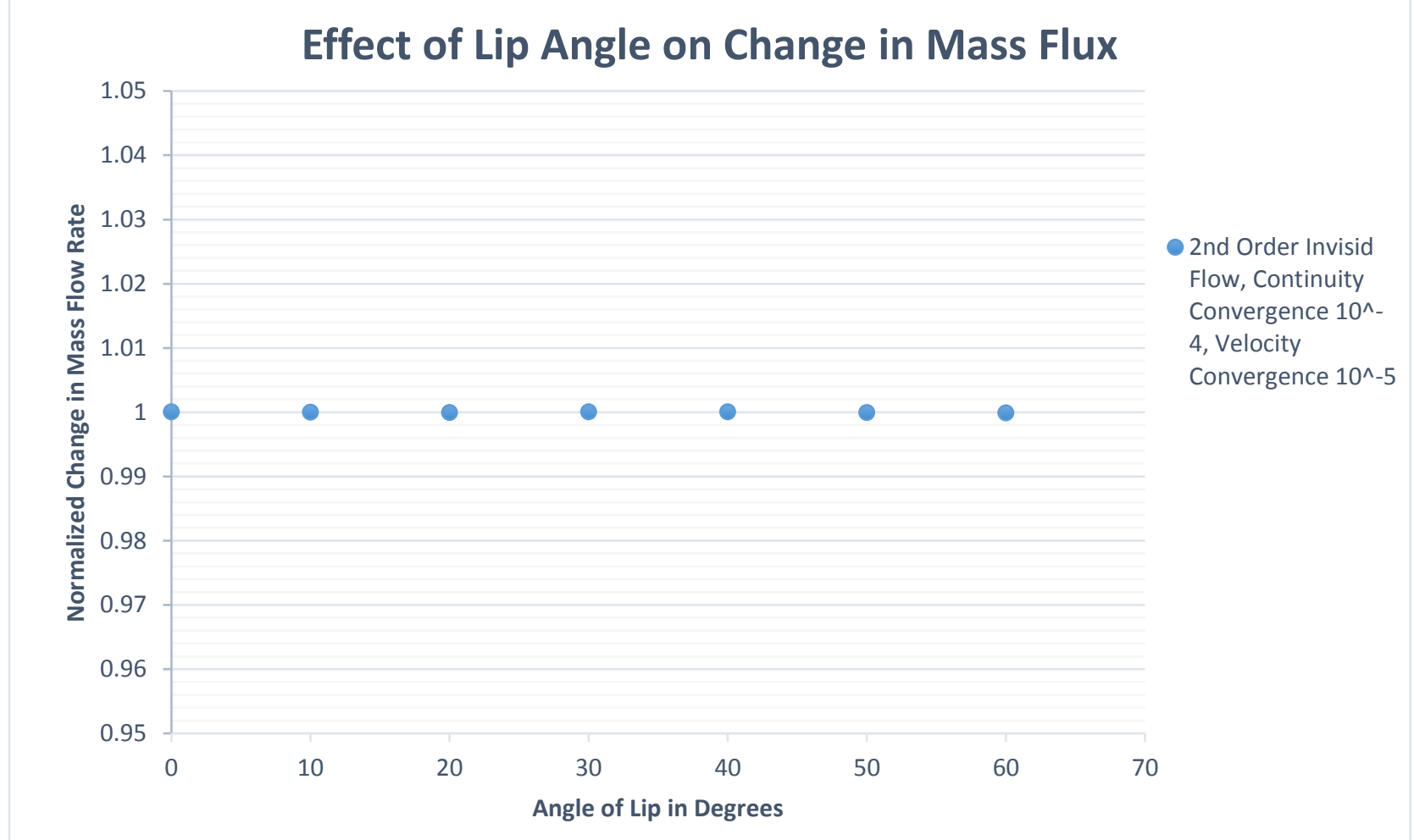

Figure 27 Effect of Lip Angle on Change in Mass Flux

The variance of the change in mass flux between inlet and outlet, $\Delta \mathrm{m}$ does not vary with the change of the lip wing, and averages around $10^{-5} \mathrm{~kg} / \mathrm{s}$, or a percentage error of $\sim 0.00001 \%$. 
This does not mean the numerical error of the simulation as a whole is so small. That cannot be inferred from the change in mass flux. However the very low error with respect to mass flux is another sign that confidence can be had in the result, and that the convergence levels achieved are producing a sufficiently accurate solution.

Having established confidence in the results, there remains the question of the exact mechanism by which the lip wing causes this increase in thrust? Going back to the thrust equation, there are two fundamental ways to increase thrust. The first is to increase the mass flow, the second is to increase the $\Delta \mathrm{V}$. The $\Delta \mathrm{V}$ can, in turn, be increased by two methods, by decreasing the inlet velocity, or increasing the outlet velocity.

Because the momentum equation is merely the mass flux multiplied by the velocity, a change in the $\Delta \mathrm{V}$ will result in a small change in mass flow. The changes in mass flux were very small fluctuations over all, generally less than $1 \%$. It's difficult to argue that this can be used as evidence, as it instinctually it falls well within the level of acceptable numerical error. This very minor fluctuation suggests change in thrust is more dependent on the change in velocity.

To verify this proposition, a second set of calculations needed to be done to calculate the inlet and outlet velocity. This proved to be somewhat of a challenge because velocity is a vector, defined for each cell at the inlet and outlet faces. The sum of the velocity across each cell is not really a metric that necessarily communicates useful information. An alternative would be to look at the mean change in velocity in the field, but this is only an approximate method. 
However, it is often a challenge to express a three-dimensional velocity field as a single numerical value. Other sources have used these methods, and so it is reasonable to use these methods to infer some conclusions, as long as their approximate nature is kept in mind.

[Anderson 3, Cummings et al.

The results of these calculations are shown in the figure below. All three show similar behaviors, an increase in the $\Delta \mathrm{V}$ the moves in step with the pattern of thrust increase. Again to ease comparison, the mean $\Delta \mathrm{V}$ at each angle is normalized against the results of the mean $\Delta \mathrm{V}$ from the shroud and disc case. 


\section{Effect of Lip Angle on Mean $\Delta \mathrm{V}$}

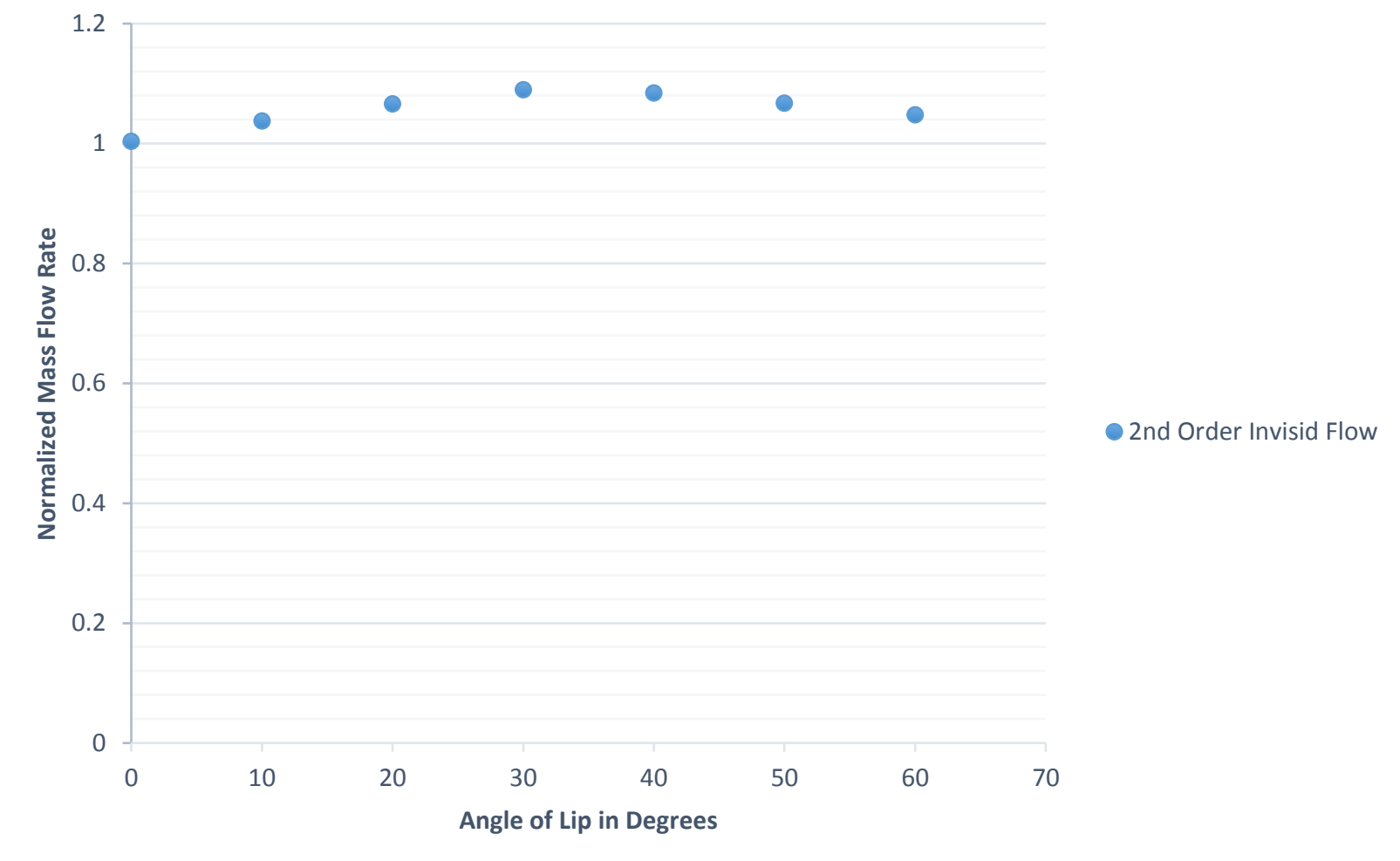

Figure 28 Effect of Lip Angle on Mean Velocity

Looking at the two graphs is should be clear that the change in mass flow is a side effect of the much larger change in velocity. This raises the question, does the change in mean $\Delta \mathrm{V}$ stem from an increase in the velocity downstream of the actuator disc, or from a decrease in velocity upstream? Or perhaps some combination of the two? 
Graphing the mean inlet and outlet velocities will again get an approximate answer to this question. As seen below, there is a minor decrease in the mean inlet velocity, and a much more pronounced increase in the wake velocity. From this can be inferred an interesting conclusion, that the upstream lip-wing via some unknown mechanism actually has a more pronounced effect downstream. Again for the sake of comparison the mean velocity has been normalized against the shroud and disc case.

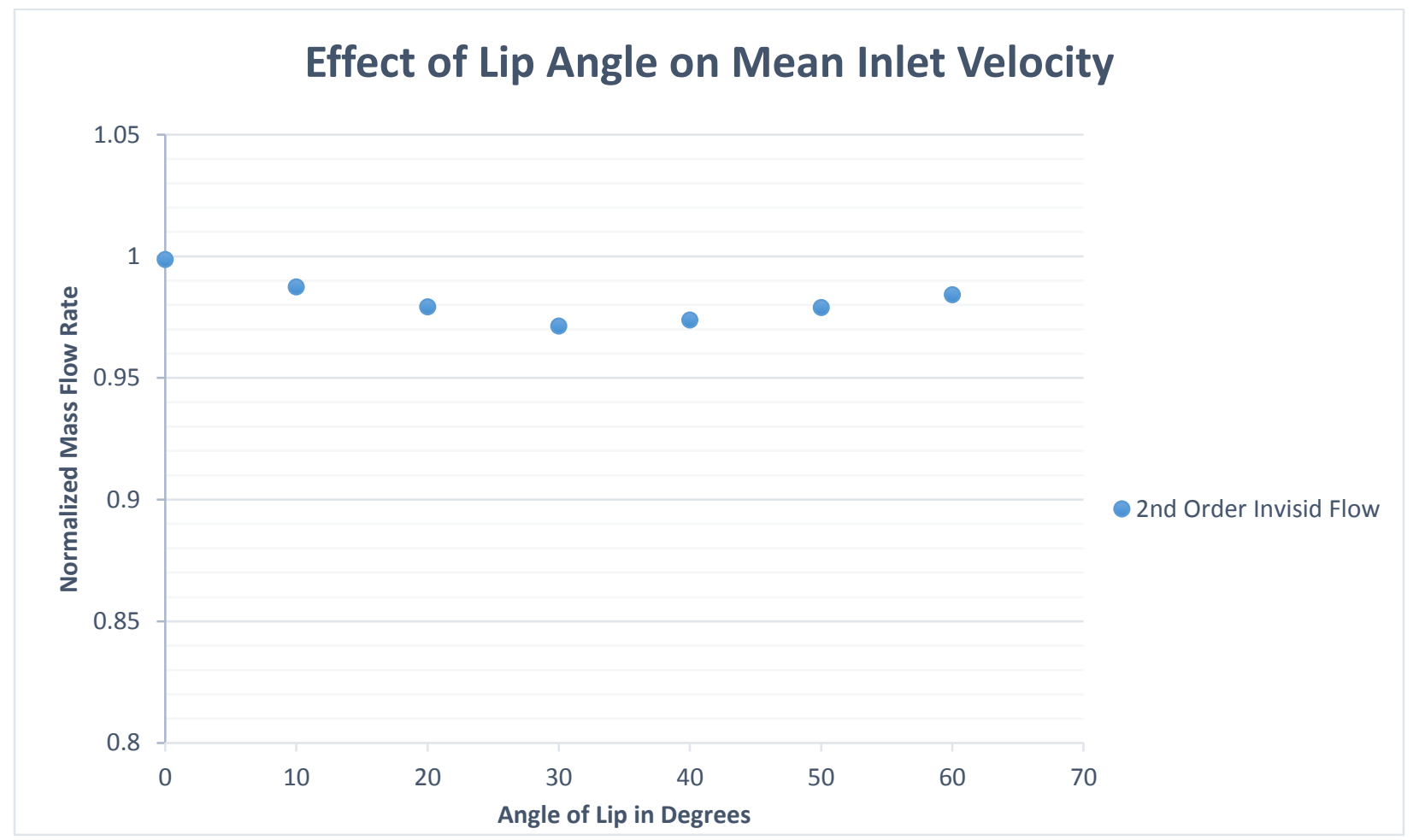

Figure 29 Mean Inlet Velocity at Different Lip Angles 


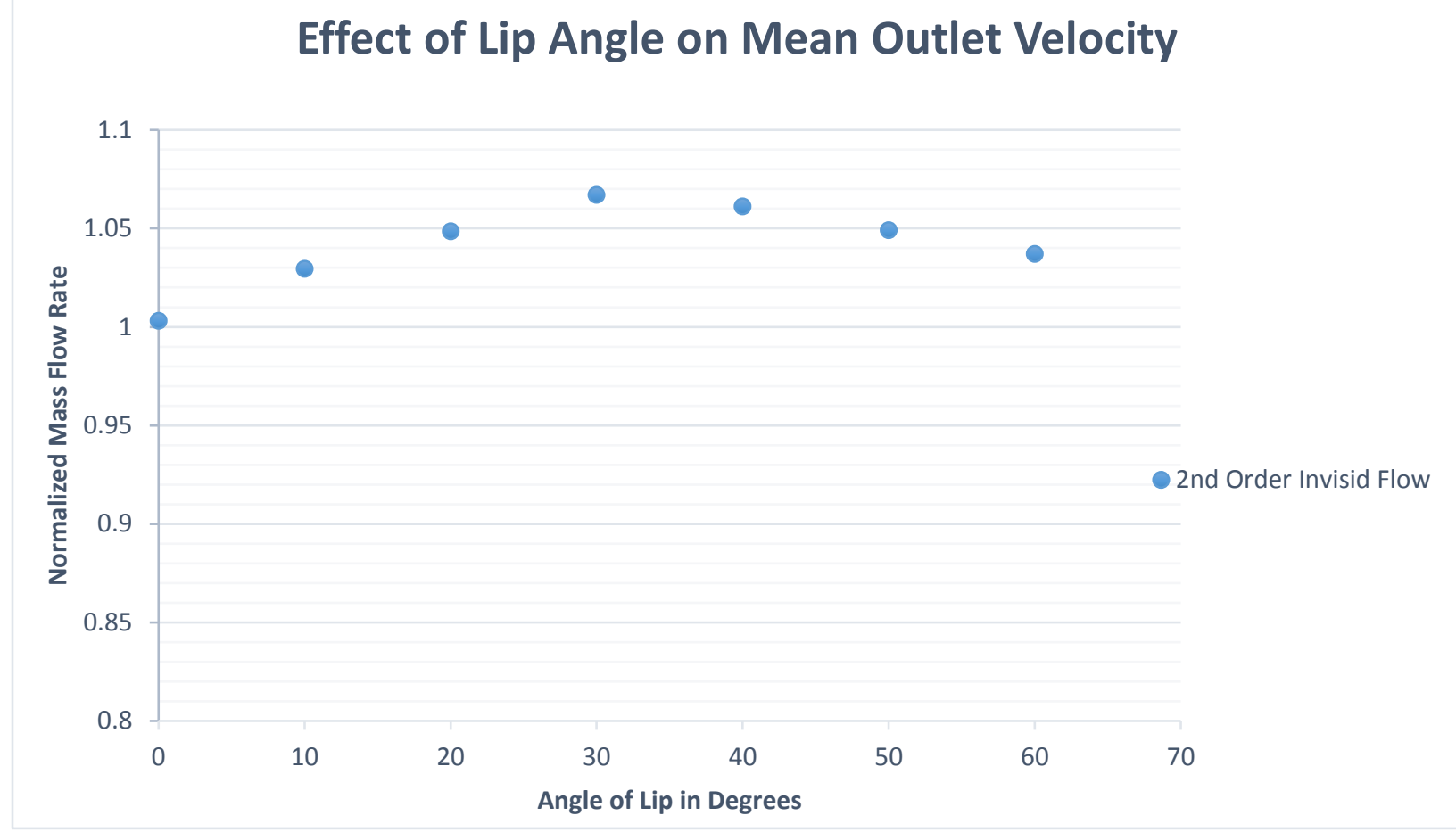

Figure 30 Mean Outlet Velocity at Different Lip Angles

In order to dive deeper into what is happening in the flow, it's necessary to look at the flow in different ways. The post processing suite from Ansys Fluent allows for contour mapping of the flow field using different variables, from temperature to molecular viscosity. Because this is a three-dimensional flow field, looking at a single plane of view risks missing important details. To counter this possibility, the following images are done with two planes, along both the $\mathrm{XY}$ and the symmetrical ZX planes. In addition, it's possible to view the streamlines of the flow in the Fluent post processing.

Looking at the dynamic pressure contours of the flow, it becomes apparent that there is a change in kinetic energy of the flow. The dynamic pressure is a very useful metric to examine, 
because it is merely the kinetic energy of the flow, scaled by the density, which remains constant. Looking at the dynamic pressure is thus the closest thing Fluent offers to examining the kinetic energy of the flow.

In the images below at the $20^{\circ}, 40^{\circ}$ and $60^{\circ}$ lip wings, the dynamic pressure follows the similar trend of the earlier figures, with a decrease in the minimum dynamic pressure, and an increase in the maximum kinetic energy. Thus it is clear that by whatever mechanism the lip wing imparts this change in thrust, it must be by changing the kinetic energy of the flow in some manner.

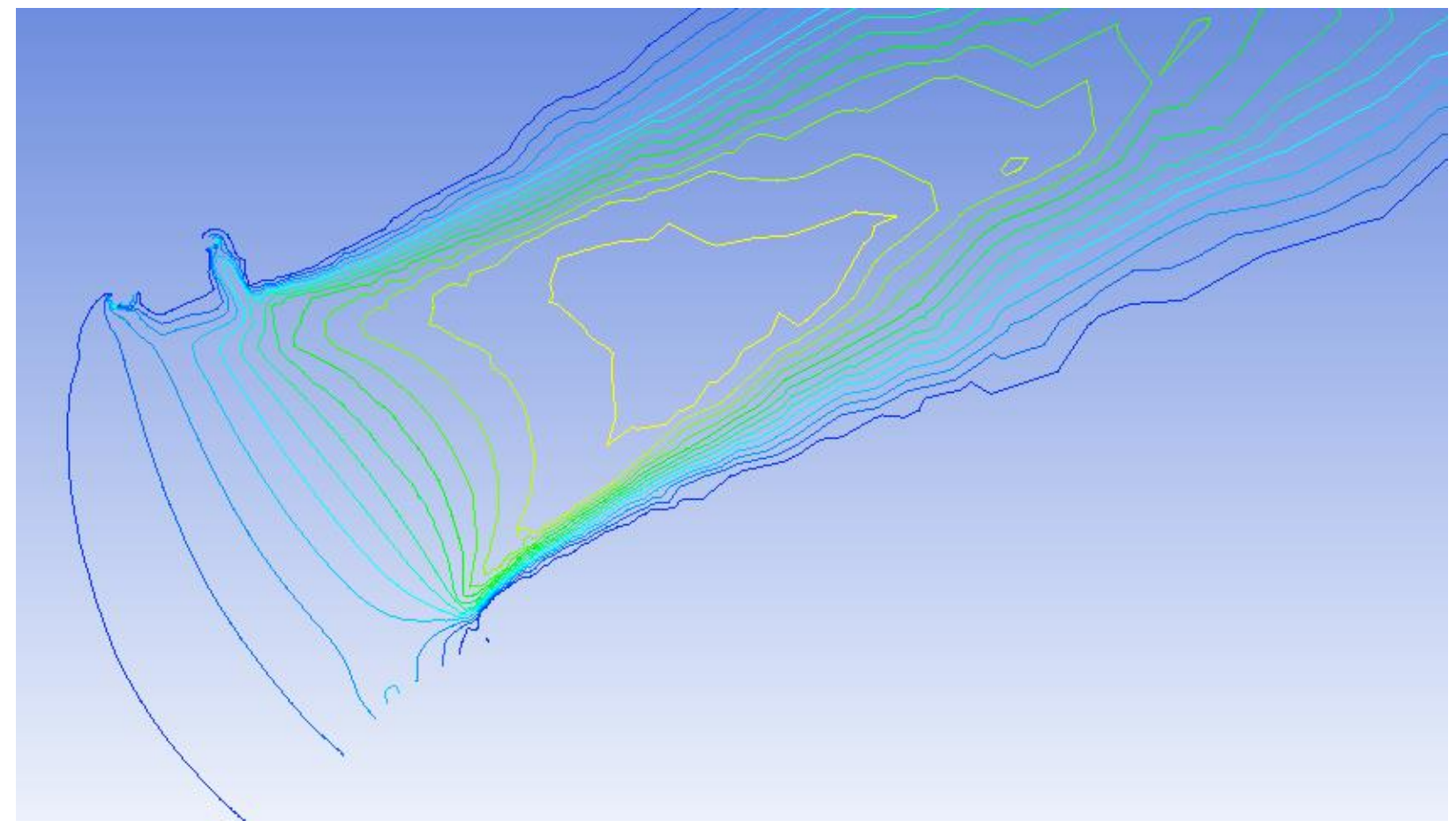

Figure 31 Dynamic Pressure Contours of Lip Wing $\left(\alpha=20^{\circ}\right)$ Ranging from 0.0017Pa to 28.765Pa 


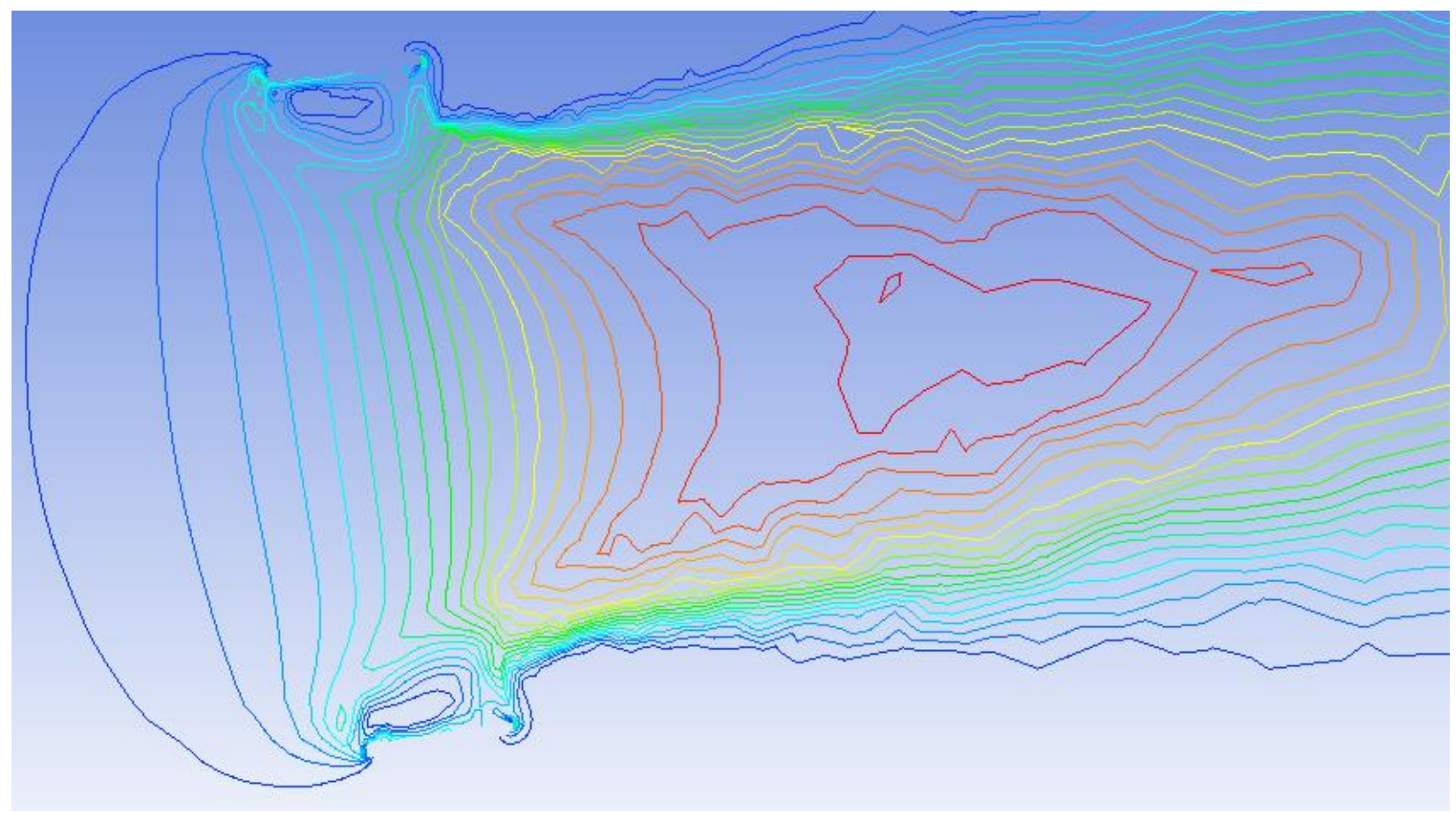

Figure 32 Dynamic Pressure Contours of Lip Wing $\left(\alpha=40^{\circ}\right)$ Ranging from 0.0013Pa to 32.168Pa

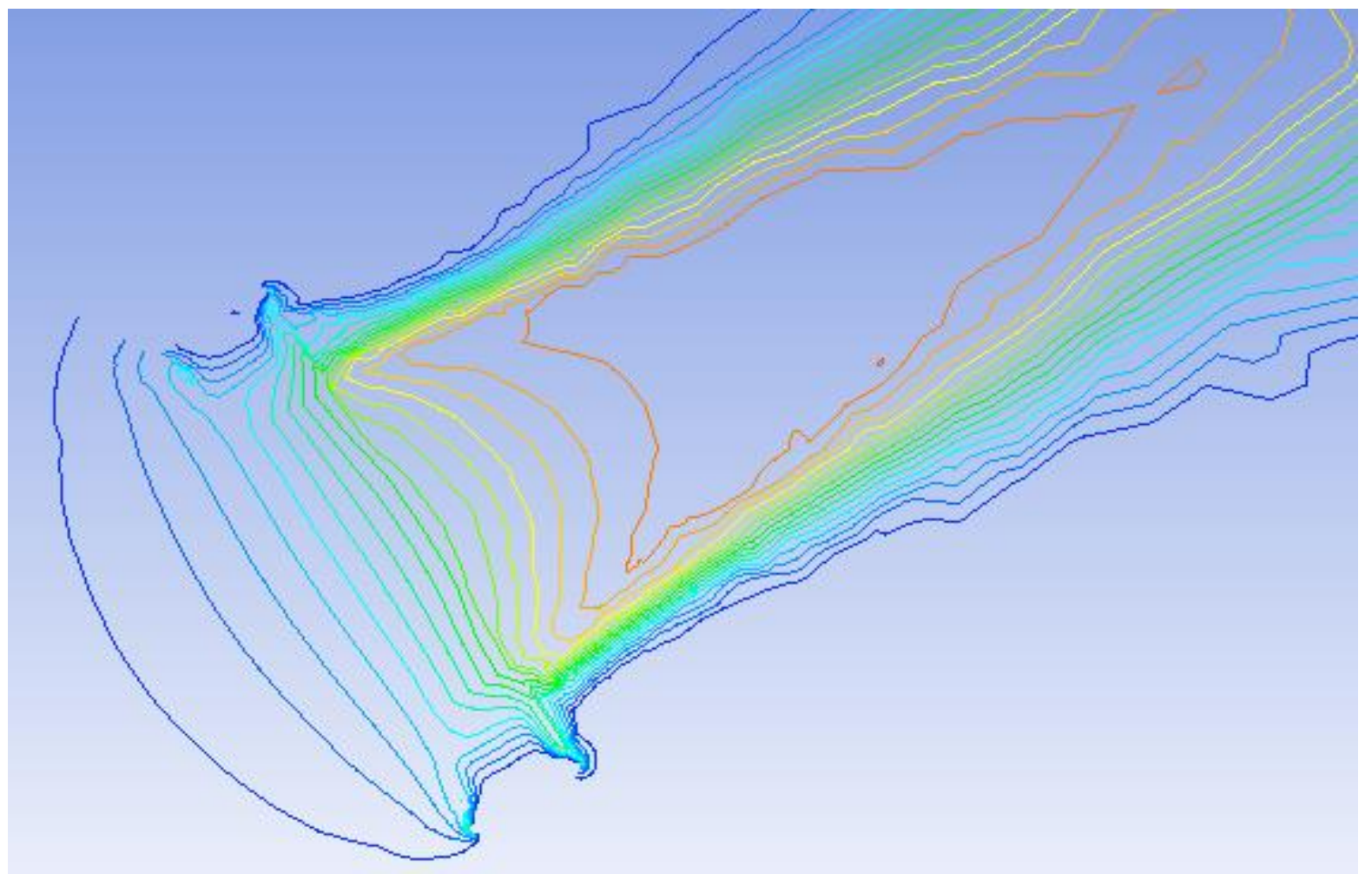

Figure 33 Dynamic Pressure Contours of Lip Wing $\left(\alpha=60^{\circ}\right)$ Ranging from 0.0014Pa to 30.341Pa 
When examining the streamlines of the various angles, it appears that the lip wing directs the flow of more streamlines towards the fan. However, it appears that at the $60^{\circ}$ degree lip angle, more of the streamlines seem to go around the lip. From this it seems reasonable to infer that the lip perhaps enlarges the capture area of the actuator disc. This is likewise supported by the decrease in the minimum velocity at the inlet. Since the mass flux is constant, as is the density, the only parameter left to vary is the effective inlet area, which would explain the decrease in minimum inlet velocity.

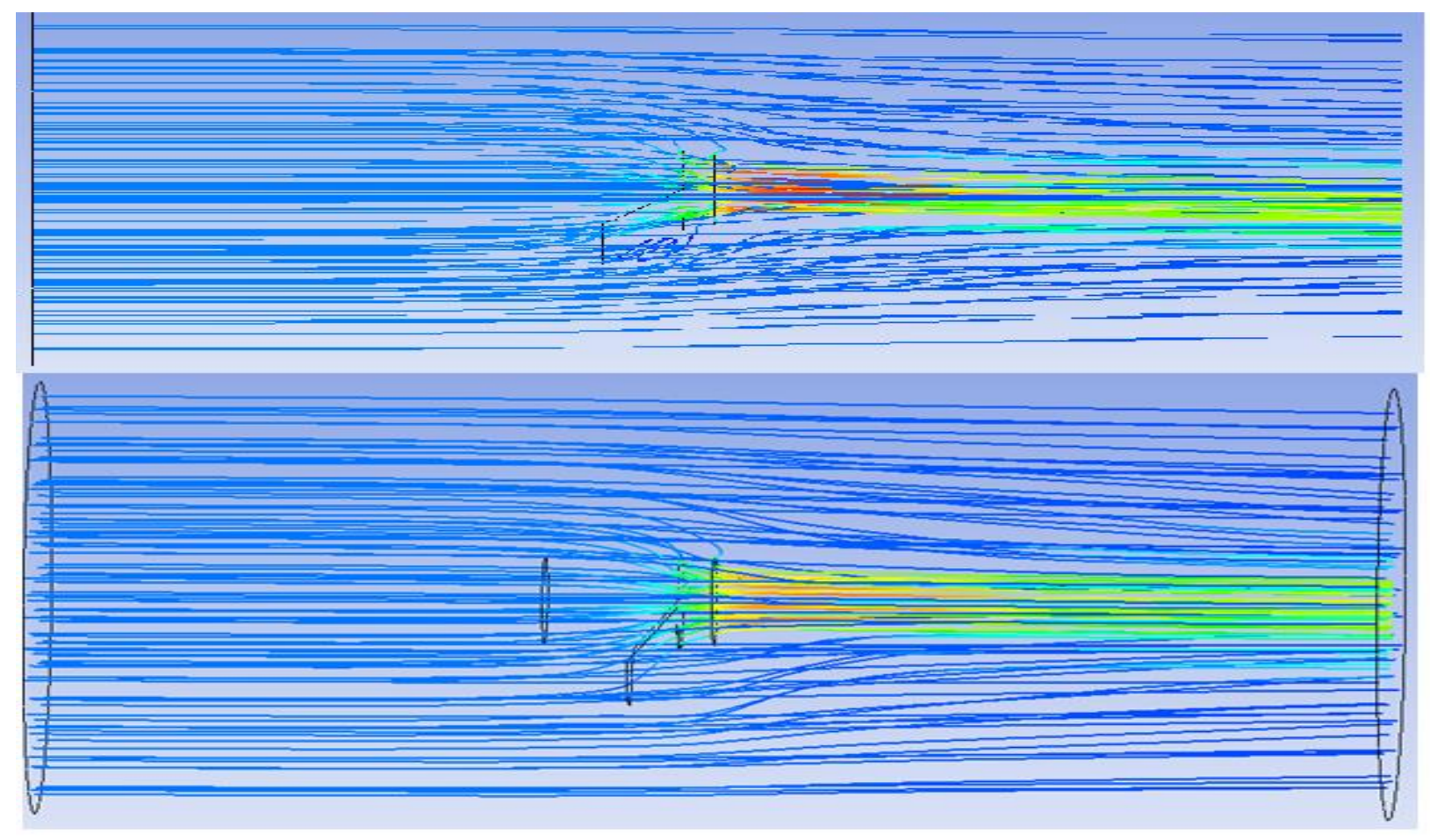

Figure 34 Comparison of Streamlines, $\alpha=40^{\circ}$ (top) and $\alpha=60^{\circ}$ (bottom)

This raises the question, is the lip wing in some way contracting the area of the wake? Examining the streamlines, it's possible that is what is occurring. Comparing the $20^{\circ}, 40^{\circ}$ and $60^{\circ}$ lip wings models, and examining only the streamlines from the actuator disc to the outlet, they 
appear to display a similar trend with respect to the change in thrust levels, i.e. that the wake seems to contract in area. Unfortunately, this is somewhat subjective and alternative explanations are plausible. Perhaps this change in area is just a trick of the viewing location, or the distribution of the starting points of the streamlines?

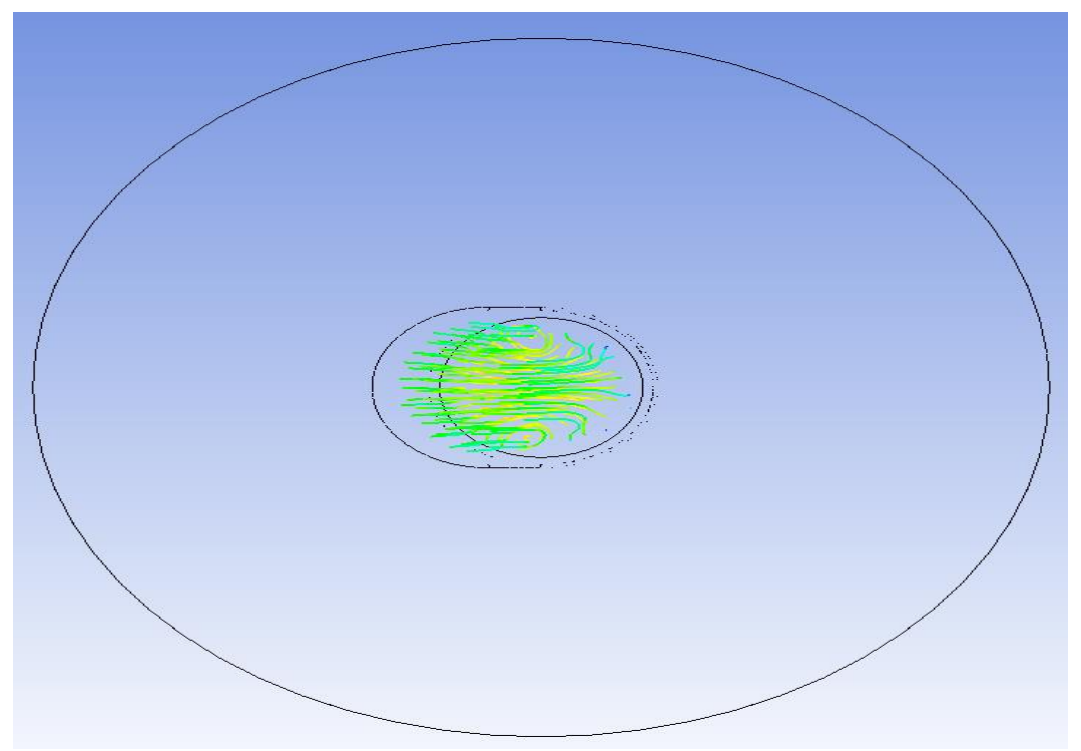

Figure 35 Streamlines Outlet, $\alpha=20^{\circ}$ 


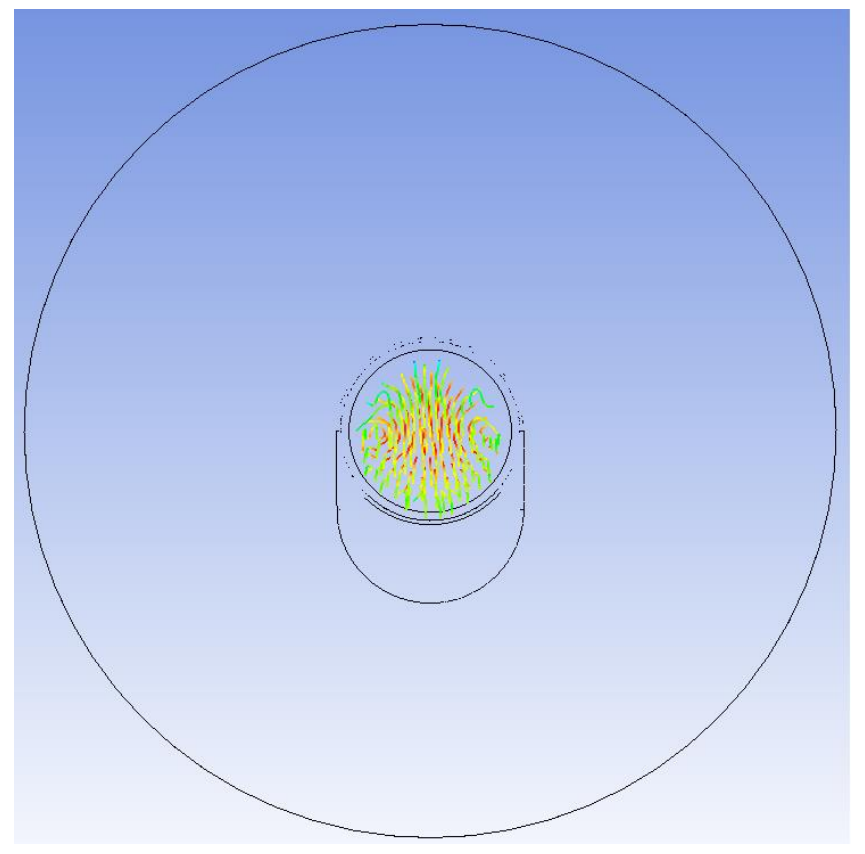

Figure 36 Streamlines Outlet, $\alpha=40^{\circ}$

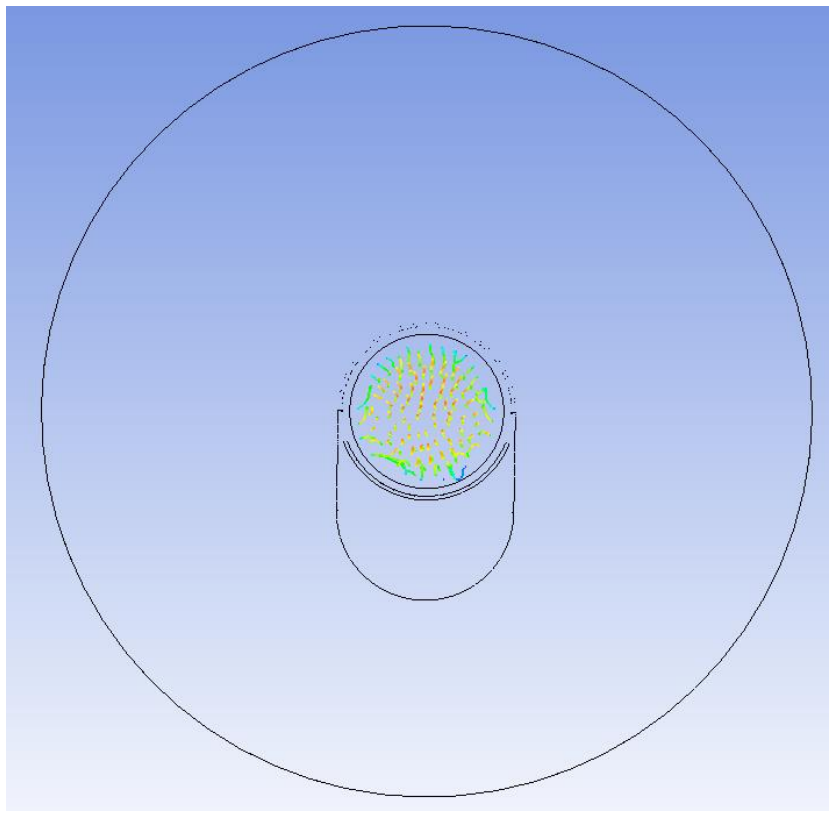

Figure 37 Streamlines Outlet, $\alpha=60^{\circ}$ 
A more conclusive is to create two rakes, lines, in Fluent, along the same planes as the dynamic pressure contour mapping, but coincident with the outlet. From the rakes, it becomes possible to graph the locations of maximum velocity flow for the various lip angles. The results of this exercise are graphed below, showing that the area of the wake is indeed decreasing in a trend similar to what is seen from the original graphs.

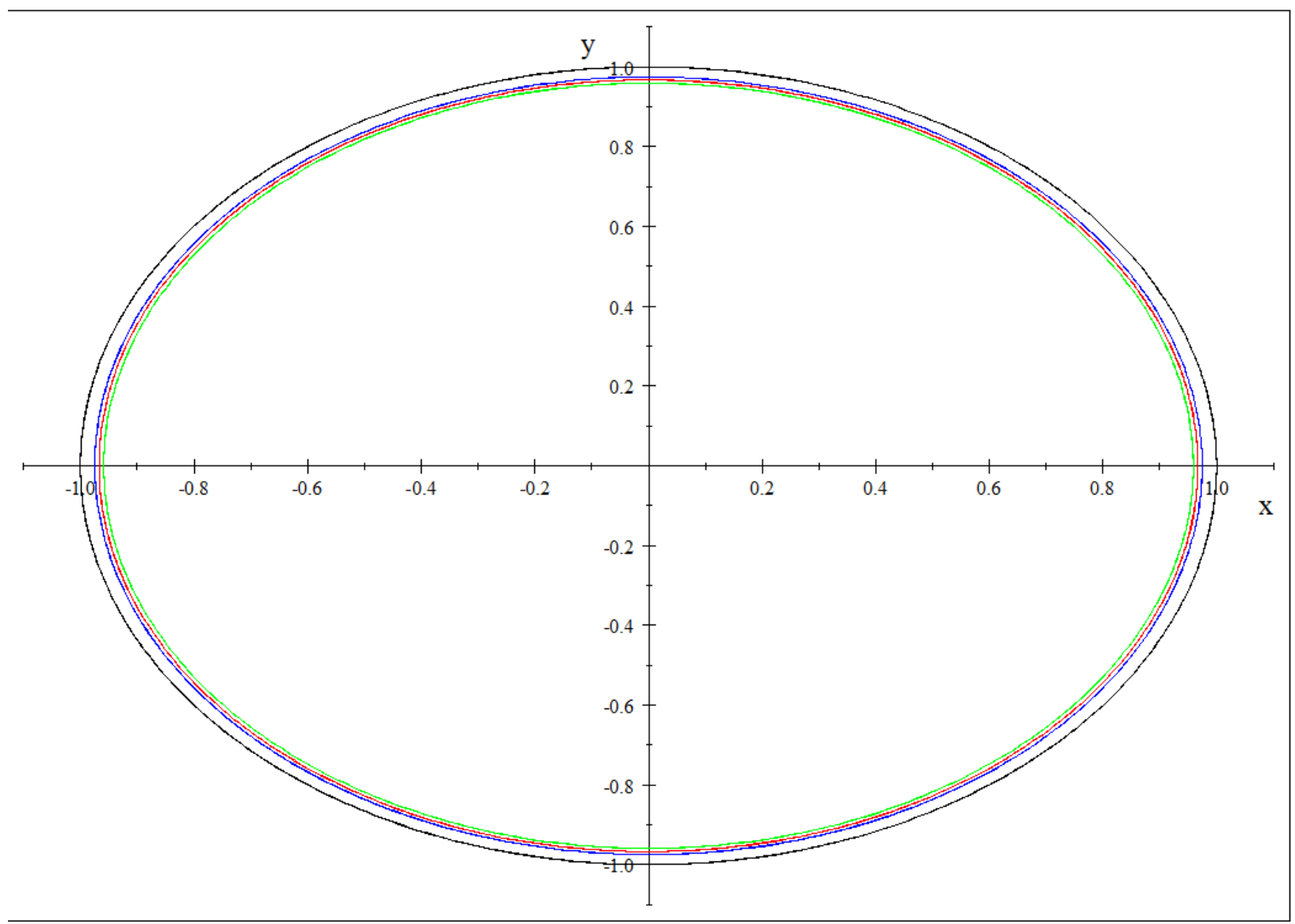

Figure 38 Normalized Areas of Max Velocity at Outlet, Black (Shroud Alone), Light Red $\left(\alpha=20^{\circ}\right)$ Light Green, $\left(\alpha=40^{\circ}\right)$ and Light Blue $\left(\alpha=60^{\circ}\right)$

When comparing the results of this model to the experimental work done by Aliptera Aircraft, some agreement can be seen between the two. Aliptera Aircraft recorded a thrust increase of $\sim 18 \%$, compared to the maximum thrust increase of $\sim 10-11 \%$ for the CFD model. 
This represents agreement in type if not strictly in magnitude. Both model and experiment agree that the lip wing will result in an increase of thrust. That experiment predicts an absolute increase $7 \%$ greater than the model is interesting. The alternative would be much easier to explain; if an inviscid model predicts higher thrust than seen in experiments, then the loss can likely be blamed on viscous or turbulent effects.

When examining the experimental apparatus, it is clear that there are several limitations given the building materials. Firstly, the lip and shroud are not a single part. This means that it is entirely possible for air to circulate around the lip and generate lift that would be incorrectly attributed to thrust. Second the building material is not completely rigid, so the aerodynamic characteristics of the structure would be in a state of flux. This is not a behavior that was modelled in Fluent so it is not entirely clear what effect it would have on the thrust. It is also clear that vibration could be a key source of experimental error. The scale used to determine the change of thrust could quite easily be influenced by the vibration of motor and structure. The type of scale used was unlikely to have any kind of vibrational dampening and so could easily confuse the sensor.

One final thought is that viscous effects really could be a source of enhancement rather than detriment to the lip wing, and maybe by including them in a more sophisticated model, the thrust enhancing properties of the lip wing would be apparent. Airfoils for example, have much lower stall angles in an inviscid flow than when laminar or turbulent effects are present due to the greater ease by which an inviscid flow can separate. Some mechanism, perhaps very similar 
in underlying principle, might be in effect for the lip wing. Perhaps viscous effects would cause a greater contraction of the flow area downstream of actuator disc. [Anderson 4]

\section{Chapter 5.0 Conclusion}

From the work in the results, it's possible to draw a few conclusions about the nature of the mechanism by which the lip wing increases the thrust. The dynamic pressure plots show that the lip wing clearly imparts some change in dynamic pressure, which is just the scaled kinetic energy, of the flow. Looking at these contour plots, it appears that the lip wing in some manner expands the difference between the minimum and maximum kinetic energy. Finally, the by examining the streamlines and graphing the area of maximum velocity in the wake, it appears as though the mechanism by which the lip wing accomplishes the change in kinetic energy is by altering the inlet capture area and constricting the area of the wake. In general, there is agreement between preliminary experimental work and the CFD model that the lip wing enhances thrust.

In this sense, perhaps the lip wing operates in a method somewhat akin to a classical nozzle, scooping up additional flow into the fan and thus enlarging and distending the capture area of the actuator disc, resulting in a higher $\Delta \mathrm{V}$ by reducing the inlet velocity and channeling the wake into a smaller area. 
This raises the question, is the lip wing itself the source of this effect by itself? Or is it the result of some manner of interaction between the lip and the actuator disc? To test this, a final 
geometry was created, with the lip separated from the shroud, as shown in the figure below.

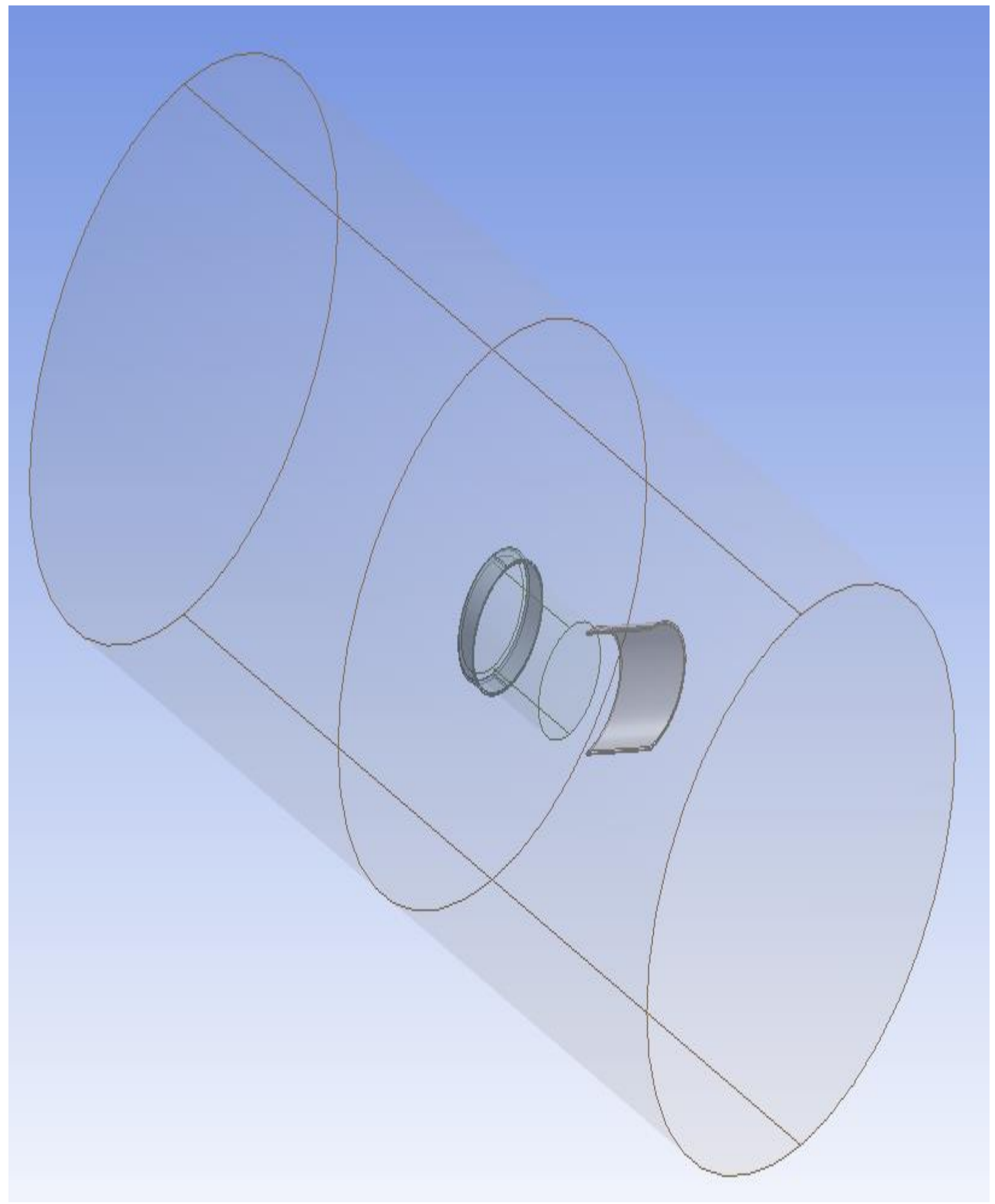

Figure 39 Geometry of Shroud and Separated Lip-Wing 
Now this situation of course is purely hypothetical. It is not possible to have the lip wing suspended in the void, with no other structural members, but that is one of the useful facets of CFD modelling, the ability to model abstract scenarios. Because the $30^{\circ}$ lip angle is the model with the greatest increase of thrust, it was selected for this alternative geometry. By modelling this scenario, it was determined that the thrust when the lip and shroud were separated by $20 \mathrm{~cm}$ was only $62 \%$ of the thrust observed when the lip and shroud were together. In addition, the streamline patterns are quite different than those formed when the two are part of a single structure, as shown below.

This would seem to be significant evidence that there is some manner of interaction between the fan and the lip wing as opposed to the lip wing itself being entirely responsible.

This preliminary work strongly suggests that there is fertile ground for further research on the lip wing concept. All the models done in this work were at approximately zero external flow, which suggests that the lip wing would at least be quite useful during the takeoff of an aircraft. However, it would also be interesting to see what effect it might have at greater speeds, during climb or cruise. 


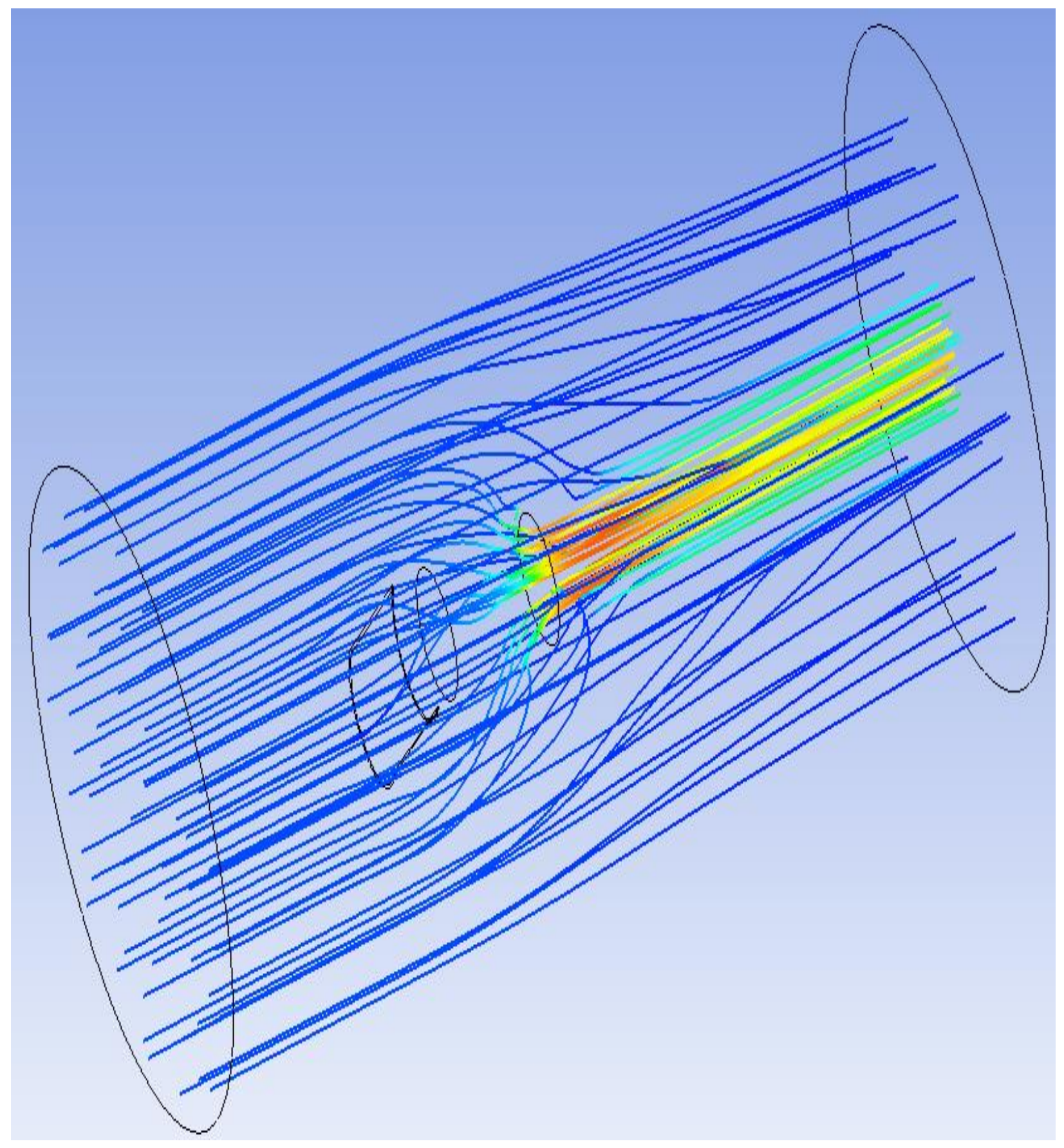

Figure 40 Streamline Diagram of Separated Lip and Shroud 
In addition, other geometries of the lip wing might produce improved effects. The length of the lip was chosen to be similar to the early experimental work but this is by no means the optimal one. It would be interesting to see if the effect improved with the increase in length or it's decrease, or perhaps using a profile shape more reminiscent of an airfoil for the lip wing.

One final issue to consider is the number of things that were omitted for simplicity. The flow modelled was inviscid and incompressible, but it might prove interesting to see what effects viscosity would have on the increase of thrust. It's possible that the viscosity might expand the capture area further at greater angles as the added friction would keep the flow attached whereas it is seen going around the lip wing when the lip angle was near its maximum however this is pure speculation.

The effect swirl might have on the model is a further matter for future consideration. It is unlikely that swirl would have had an identifiable effect in an inviscid model, as without a viscous loss, the swirl should simply have gone on to infinity, and any deviation from this would simply have been numerical error. With viscosity included however, the swirl momentum would eventually decay at a certain distance upstream and downstream of the disc. What effect this might have on the capture area or the downstream area of the actuator disc is not clear, but potentially worth investigating.

Of course, for a really in-depth analysis, it would be necessary to replace the actuator disc with a full propeller model. This would necessitate a much more computationally expensive 
analysis, using a dynamic mesh and a transient solver, with verification work in wind tunnels.

This would add a host of new variables for in depth analysis and optimization of the design. Such work however, must be left to future researchers. 


\section{References}

Anderson, John David. A history of aerodynamics and its impact on flying machines.

Cambridge, Cambridge University Press, 1999. [Anderson 1]

Anderson, John David. Aircraft Design and Performance. Cambridge, Cambridge University

Press, 1999. [Anderson 2]

Anderson, John David. Computational Fluid Dynamics: Basics and Applications. Cambridge, Cambridge University Press, 2005. [Anderson 3]

Anderson, John David. Fundamentals of Aerodynamics, $4^{\text {th }}$ Edition, Cambridge, Cambridge University Press, 2003. [Anderson 4]

Greatrix, David. Powered Flight, The Engineering of Aerospace Propulsion. London, SpringerVerlag. 2012

ANSYS Inc, Ansys Fluent and User Guide, 12 Edition, (C2009

Carlton, John. Marine Propellers and Propulsion, $3^{\text {rd }}$ Edition London, Springer-Verlag. 2012

Gunston, Bill, The Cambridge Aerospace Dictionary Cambridge, Cambridge University Press 2004 ,

F. Marc de Piolenc, George E. Wright, Jr. Ducted Fan Design, Volume 1: Volume 1 - Propulsion Physics and Design of ... 
Aagaard Madsen, H. “A CFD Analysis of the Actuator Disc Flow Compared with Momentum Theory Results.” Ed. B. Maribo Pedersen. Iea Joint Action. Aerodynamics of Wind Turbines (1997): 109-124. Print.

Madsen, H.A., Mikkelsen, R., Øye, S., Bak, C., and Johansen, J., 2007, “A Detailed Investigation of the Blade Element Momentum (BEM) model based on analytical and numerical results and proposal for modification of the BEM model," The Science of Making Torque from Wind, Journal of Physics: Conference Series 75

Simms, D., Schreck, S., Hand, M., and Fingersh, L.J., 2001, "NREL Unsteady Aerodynamics Experiment in the NASA-Ames Wind Tunnel: A Comparison of Predictions to Measurements," Technical report for the National Renewable Energy Laboratory, TP-500-29494

Mikkelsen, R., 2003, “Actuator Disk Methods Applied to Wind Turbines,” Dissertation submitted to Technical Uni. of Denmark, Fluid Mechanics, Dept. of Mech. Eng.

David Hartwanger and Dr Andrej Horvat, 3D Modelling of a Wind Turbine Using CFD, NAFEMS Conference 2008

D. J. Sharpe, A General Momentum Theory Applied to an Energy-extracting Actuator Disc, Wind Energ. 2004; 7:177-188

Van Kuik GAM. On the limitations of Froude's actuator disc concept. PhD Thesis, Technical University of Eindhoven,

1991. 
Ammara I, Leclerc C, Masson C. A Viscous Three-Dimensional Differential/Actuator-Disk Method for the Aerodynamic Analysis of Wind Farms. ASME. J. Sol. Energy Eng. 2002;124(4):345-356

Crespo, A., Manuel, F., Moreno, D., Fraga, E., and Hernandez, J., 1985, “Numerical Analysis of Wind Turbine Wakes," Workshop on Wind Energy Applications, Delphi, Greece, pp. 15-25.

W. M. J. Batten1, M. E. Harrison and A. S. Bahaj1, Accuracy of the actuator disc-RANS approach for predicting the performance and wake of tidal turbines, Philosophical Transactions of the Royal Society, January 2013

Harrison ME, BattenWMJ, Bahaj AS. 2010 A blade element actuator disc approach applied to tidal stream turbines. In Proc. OCEANS 2010, Seattle, WA, USA.

Frédéric Le Chuiton, Actuator disc modelling for helicopter rotors, Aerospace Science and Technology 8 (2004) 285-297

N. Bettschart, Rotor fuselage interaction: Euler and Navier-Stokes computations with an actuator disk, in: Proceedings of the American Helicopter Society, 55th Annual Forum, Montreal, Canada, 1999.

Eric W. M. Roosenboom, Arne Stürmer, and Andreas Schröder, Advanced Experimental and Numerical Validation and Analysis of Propeller Slipstream Flows, Journal of Aircraft 2010 47:1, 284-291

Oberkampf, W. L., and Trucano, T. G., "Verification and Validation in Computational Fluid Dynamics," Progress in Aerospace Sciences, Vol. 38, 2002, pp. 209-272. 
Marvin, J. G., "Accuracy Requirements and Benchmark Experiments for CFD Validation," NASA Rept. TM-100087, 1988.

Peter M. Goorjian, An Invalid Equation in the General Momentum Theory of the Actuator Disk, AIAA Journal, VOL. 10, NO. 41972

J. N. Sùrensen, W. Z. Shen and X. Munduate, Analysis of Wake States by a Full-Field Actuator Disc Model, WIND Wind Energ., 1, 73-88 (1998)

T. Y. Wu, `Flow through a heavily loaded actuator disc', Schistechnik, 9, 134-138 (1962).

I-Chung Chang, R. G. Rajagopalan, CFD Analysis for Ducted Fans with Validation, 21st Applied Aerodynamics Conference 23-26 June 2003

Rajagopalan R.G. and Mathur S. Three dimensional analysis

of a rotor in forward flight. Journal of the American Helicopter Society, 38:14-25, 1993

Jie Zhao, Qingming Hou, Hongzhe Jin, Yanhe Zhu, and Ge Li. CFD Analysis of Ducted-Fan UAV Based on Magnus Effect Proceedings of 2012 IEEE International Conference on Mechatronics and Automation August 5 - 8, Chengdu, China

Chang, I. C. and Rajagopalan, R. G. (2003). Cfd analysis for ducted fans with validation. 21th AIAA Applied Aerodynamics Conference.

He, C. and Xin, H. (2006). An unsteady ducted fan model for rotorcraft flight simulation. 62th AHS Forum.

Ko, A., Ohanian, O. J., and Gelhausen, P. (2007). Ducted fan uav modeling and simulation in preliminary design. AIAA Modeling and Simulation Technologies Conference and Exhibit. 
J.-M. Bousquet, P. Gardarein. Improvements on computations of high speed propeller unsteady aerodynamics. Aerospace Science and Technology 7 (2003) 465-472

W. Shawn Westmoreland, Robert W. Tramel, and Jennie Barber. Modeling Propeller FlowFields Using CFD, 46th AIAA Aerospace Sciences Meeting and Exhibit

F. Moens, P. Gardarein, Numerical Simulation of the Propeller/Wing Interactions For Transport Aircraft, $19^{\text {th }}$ Applied Aerodynamics Conference.

Osgar John Ohanian III, 2011. Ducted Fan Aerodynamics and Modeling, with Applications of Steady and Synthetic Jet Flow Control, Dissertation, Virginia Polytechnic Institute and State University.

Edward F Blick and Vincent Homer, 1971 Power-on Channel Wing Aerodynamics, Journal of Aircraft vol 8 number 40

Riccardo Brogliaa, Giulio Dubbiosoa, Danilo Durantea, Andrea Di Masciob. Simulation of turning circle by CFD: Analysis of different propeller models and their effect on manoeuvring prediction. Applied Ocean Research 39 (2012) 1-10

J.N. Sørensen and A. Myken. Unsteady actuator disc model for horizontal axis wind turbines. J. Wind Eng. Ind. Aerodyn., 39:139-149, 1992.

James DeSpirito, Subsonic Flow CFD Investigation of Canard Controlled Missile with Planar and Grid Fins. 41st Aerospace Sciences Meeting and Exhibit 6-9 January 2003,

Wen Zhong Shen, Jens Nørkær Sørensen, Robert Mikkelsen, Tip Loss Correction for Actuator/Navier-Stokes Computations. Journal of Solar Energy EngineeringVol. 127, May 2005 
Sørensen, J. N., and Shen, W. Z., 2002, “Numerical Modelling of Wind Turbine Wakes,' J. Fluids Eng., 124, pp. 393-399.

Snel, H., and Van Holten, T., 1995, “Review of Recent Aerodynamic Research on Wind Turbines With Relevance to Rotorcraft," AGARD report, CP-552, Chap. 7.

Martin O. L. Hansen, Jeppe Johansen Tip Studies Using CFD and Comparison with Tip Loss Models Wind Energy. 2004; 7:343-356

Arne Stuermer. Unsteady CFD Simulations of Contra-Rotating Propeller Propulsion Systems, 44th AIAA/ASME/SAE/ASEE Joint Propulsion Conference \& Exhibit 21 - 23 July 2008

Arne W. Stuermer, Unsteady CFD Simulations of Propeller Installation Effects, 42nd AIAA/ASME/SAE/ASEE Joint Propulsion Conference \& Exhibit 9 - 12 July 2006

4Stuermer, A., "Validation of an Unstructured Chimera Grid Approach for the Simulation of Propeller Flows,” Tech. Rep. AIAA 2004-5289, 2004.

Réthoré, P-E. M., Sørensen, N. N., \& Zahle, F. (2010). Validation of an Actuator Disc Model. In EWEC 2010

Proceedings online. European Wind Energy Association (EWEA).

B. D. Goble and J. R. Hooker, Validation of an Unstructured Grid Euler/Navier-Stokes Code on a Full Aircraft with Propellers. 39th AIAA Aerospace Sciences

Meeting \& Exhibit 8-11 January 2001

G. Crastoa*, A.R. Gravdahla, F. Castellanib, E. Piccionib.

Wake modeling with the Actuator Disc concept Energy Procedia 24 ( 2012 ) 385 - 392 
Crasto G, Gravdahl AR. CFD wake modeling using a porous disc. EWEC 2008 Proceedings, Brussels, Belgium.

Crasto G, Castellani F, Gravdahl AR, Piccioni E. Wind Turbines Wakes Calculations:

Comparisons Of Actuator Disc

Against Analytical Models. AWEA 2011 WindPower Proceedings, Anaheim, California.

Stan, Dusan, Alipetra Aircraft Overview, http://aliptera.com, Last Accessed August 25, 2016

J.H Ferziger, M. Peric. Computational Method for Fluid Dynamics, $3^{\text {rd }}$ Edition, Springer-Verlag, Berlin, 2002

Cumming, Russel. Mason, William. Morton, Scott and McDaniel, David. Applied Computational Aerodynamics. Cambridge Aerospace Series, Cambridge, 2015.

NACA Research Memorandum, Langley Full Scale Tunnel Tests of the Custer Channel Wing Airplane, (L53A09) April 7, 1953. 